\title{
Heterotic-F-theory duality with Wilson line symmetry-breaking
}

\author{
Herbert Clemens ${ }^{a}$ and Stuart Raby ${ }^{b}$ \\ ${ }^{a}$ Mathematics Department, Ohio State University, \\ Columbus, OH 43210, U.S.A \\ ${ }^{b}$ Physics Department, Ohio State University, \\ Columbus, OH 43210, U.S.A \\ E-mail: clemens.43@osu.edu, raby.1@osu.edu
}

ABSTRACT: We begin with an $E_{8} \times E_{8}$ Heterotic model broken to an $\mathrm{SU}(5)_{\text {gauge }}$ and a mirror $\mathrm{SU}(5)_{\text {gauge, }}$ where one $\mathrm{SU}(5)$ and its spectrum is identified as the visible sector while the other can be identified as a hidden mirror world. In both cases we obtain the minimal supersymmetric standard model spectrum after Wilson-line symmetry-breaking enhanced by a low energy R-parity enforced by a local (or global) U(1) $X$-symmetry. Using Heterotic/F-theory duality, we show how to eliminate the vector-like exotics which were obtained in previous constructions. In these constructions, the Calabi-Yau [CY] four-fold was defined by an elliptic fibration with section over a base $B_{3}$ and a GUT surface given by $K 3 / \mathbb{Z}_{2}=$ Enriques surface. In the present paper we construct a quotient CY four-fold fibered by tori with two elliptic structures given by a pair of sections fibered over the Enriques surface. Using Heterotic/F-theory duality we are able to define the cohomologies used to derive the massless spectrum.

Our model for the 'correct' $F$-theory dual of a Heterotic model with Wilson-line symmetry-breaking builds on prior literature but employs the stack-theoretic version of the dictionary between the Heterotic semi-stable $E_{8}$-bundles with Yang-Mills connection and the $d P_{9}$-fibrations used to construct the $F$-theory dual.

KeYwords: Differential and Algebraic Geometry, F-Theory, Supersymmetric Gauge Theory

ARXIV EPRINT: 1908.01913 


\section{Contents}

1 Introduction 1

1.1 The physics 1

1.2 The mathematics 3

1.3 The organization of the paper 5

$2 W_{4} / B_{3}, E_{8}$-unfolding and its symmetries $\quad 6$

2.1 Weierstrass form with respect to $\zeta \quad 7$

2.2 The second section 8

$\begin{array}{lr}2.3 \text { The quotient Calabi-Yau manifolds } & 10\end{array}$

$\begin{array}{ll}2.4 \text { Three Calabi-Yau fourfolds related by quotienting } & 10\end{array}$

2.5 Unfolding the $E_{8}$-singularity 11

3 The spectral divisor $\quad \mathbf{1 2}$

$\begin{array}{ll}3.1 \text { Adjusting the } E_{8} \text {-evolution } & 15\end{array}$

4 Semi-stable degeneration and $V_{3} / B_{2} \quad 16$

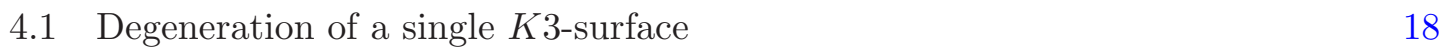

$\begin{array}{lll}4.2 & d P_{9} \text {-bundles over } B_{2} \text { giving a singular Calabi-Yau fourfold } & 19\end{array}$

$\begin{array}{ll}4.3 \text { The involution } & 21\end{array}$

5 Passing from Heterotic theory to F-theory 22

5.1 Initial data 22

5.2 Building a normal-crossing $K 3$ from an elliptic curve with two flat $E_{8}$-bundles 23

5.3 The action of the involution on the Heterotic model 24

$\begin{array}{lll}5.4 & \mathrm{SU}(5)_{\text {gauge-roots }} \text { and the semi-stable limit } & 27\end{array}$

6 Geometric model-double cover form $\quad 28$

6.1 Putting the sections $\zeta$ and $\tau$ of $W_{4} / B_{3}$ on equal footing 28

6.1.1 Line between the sections 28

6.2 Fundamental projection and modified Weierstrass form 29

$\begin{array}{ll}6.3 \text { The branch locus } & 31\end{array}$

6.4 The standard $\mathbb{P}^{112}$-formulation 31

7 Desingularization of $\bar{W}_{4} \quad 32$

7.1 Localizing at the singularities of $\bar{W}_{4} \quad 32$

7.2 Resolving the singularities of $\bar{W}_{4}$

$\begin{array}{lll}\text { 7.2.1 } & \text { First modification of } \Delta & 33\end{array}$

$\begin{array}{lll}7.2 .2 & \text { Second modification of } \Delta & 34\end{array}$

$\begin{array}{ll}\text { 7.2.3 Singularities of higher codimension } & 36\end{array}$

$\begin{array}{lll}7.3 & \text { The smooth model } \tilde{W}_{4} & 37\end{array}$

7.4 The spectral divisor in $\tilde{W}_{4} \quad 38$

$\begin{array}{lll}\text { 7.4.1 Locating matter curves and the Higgs curve } & 39\end{array}$ 
$\begin{array}{ll}\text { 7.5 Topology of } \tilde{W}_{4} & 40\end{array}$

$\begin{array}{lll}\text { 7.5.1 Picard groups } & 40\end{array}$

7.5.2 Intersections in $Q^{(2)} \quad 41$

8 Higgs line bundle and the $G$-flux $\quad 42$

$\begin{array}{lll}8.1 & \text { Physical interpretation } & 42\end{array}$

8.2 The Higgs bundle 43

8.3 Restrictions to the matter and Higgs curves 44

8.4 Action of $\tilde{\beta}_{4}$ on $\mathcal{L}_{\text {Higgs }} \quad 46$

8.5 The $G$-flux in $\tilde{W}_{4} \quad 46$

8.6 Numerical conditions on the square of the $G$-flux 47

$\begin{array}{ll}8.7 & \text { The } D \text {-term }\end{array}$

9 Wilson line: symmetry-breaking to the standard model 48

10 Bulk and chiral spectra with Wilson Line on $W_{4}^{\vee} \quad 50$

$\begin{array}{ll}10.1 \text { Bulk spectrum with Wilson line } & 50\end{array}$

$\begin{array}{ll}10.2 \text { Matter spectrum } & 52\end{array}$

11 Asymptotic $\mathbb{Z}_{4}$ R-symmetry

12 Conclusions $\quad 54$

A $\mathbb{P}^{112}$ Formulations by Sakura Schäfer-Nameki $\quad \mathbf{5 4}$

A.1 Realizing $\bar{W}_{4}$ in $\mathbb{P}^{112} \quad 54$

$\begin{array}{lll}\text { A.2 } & \text { Other models with similar fiber type } & 56\end{array}$

\section{Introduction}

\subsection{The physics}

Supersymmetric grand unified theories [SUSY GUTs] [17, 18, 29] have many nice properties. These include an explanation of the family structure of quarks and leptons with the requisite charge assignments under the Standard Model $[\mathrm{SM}]$ gauge group $\mathrm{SU}(3)_{C} \times \mathrm{SU}(2)_{L} \times \mathrm{U}(1)_{Y}$ and a prediction of gauge coupling unification at a scale of order $10^{16} \mathrm{GeV}$. The latter is so far the only direct hint for the possible observation of supersymmetric particles at the LHC. UV completions of SUSY GUTs in string theory also provide a consistent quantum mechanical description of gravity. As a result of this golden confluence, many groups have searched for SUSY GUTs in string theory. In fact, it has been shown that by demanding SUSY GUTs in string constructions one can find many models with features much like that of the minimal supersymmetric Standard Model [MSSM] [2, 3, 8, 32, 36-38].

The past several years have seen significant attention devoted to the study of supersymmetric GUTs in $F$-theory [5, 6, 9, 20, 21, 24, 52]. Both local and global SU(5) $F$-theory GUTs have been constructed where SU(5) is spontaneously broken to the SM via 
non-flat hypercharge flux. One problem with this approach for GUT breaking is that large threshold corrections are generated at the GUT scale due to the non-vanishing hypercharge flux $[9,10,21,44]$. An alternative approach to breaking the GUT group is using a Wilson line in the hypercharge direction, i.e. a so-called flat hypercharge line bundle. In this case it is known that large threshold corrections are not generated at the GUT scale (or, in fact, leading to precise gauge coupling unification at the compactification scale in orbifold GUTs) [33, 49] and [1, 27, 50, 53].

In a previous paper, the present authors and collaborators constructed a global SU(5) $F$-theory model with Wilson line breaking [43]. The model contained the vector multiplets for the MSSM gauge group, 3 families of quarks and leptons, 4 pairs of Higgs doublets, and in addition, a vector-like pair of chiral multiplets in the representation $(\mathbf{3}, \mathbf{2})_{-5 / 6} \oplus$ $(\overline{\mathbf{3}}, \mathbf{2})_{+5 / 6}$. In terms of the model defined on an elliptically fibered CY 4-fold with GUT surface defined as an Enriques surface, $K_{3} / \mathbb{Z}_{2}$, the massless spectrum is given in terms of cohomologies of the flux line bundle (or twists of the flux line bundle) on the GUT surface. It was then shown that the holomorphic Euler character of any flat bundle on $S_{\mathrm{GUT}}$ is equivalent to its Todd genus [6,20,43], through

$$
c_{1}\left(L_{Y}\right)=0 \quad \Longrightarrow \quad \chi\left(S_{2}, L_{Y}\right)=\int_{S_{\mathrm{GUT}}} \mathrm{Td}\left(T S_{\mathrm{GUT}}\right)
$$

so we have that

$$
\chi\left(S_{\mathrm{GUT}}, L_{Y}\right)=h^{0}\left(S_{\mathrm{GUT}}, L_{Y}\right)-h^{1}\left(S_{\mathrm{GUT}}, L_{Y}\right)+h^{2}\left(S_{\mathrm{GUT}}, L_{Y}\right)=1 .
$$

Since all $h^{m}\left(S_{\mathrm{GUT}}, L_{Y}\right)$ cannot be vanishing, we are guaranteed to get some massless vector or chiral states, $(\mathbf{3}, \mathbf{2})_{-5 / 6}$ 's and $(\overline{\mathbf{3}}, \mathbf{2})_{+5 / 6}$ 's. We emphasize that the presence of some kind of vector-like exotic matter is not a specific issue with this Enriques model but rather a general property of any model that breaks $\mathrm{SU}(5)_{\text {GUT }} \rightarrow \mathrm{SU}(3) \times \mathrm{SU}(2) \times \mathrm{U}(1)_{Y}$ with a flat $\mathrm{U}(1)_{Y}$ bundle on a holomorphic surface $S_{\mathrm{GUT}}$. We note that this derivation is, however, only valid if the $F$-theory is compactified on a $\mathrm{CY} 4$-fold with section. In the present paper we show how to eliminate the vector-like exotics and evade this theorem.

We need to understand how to modify the description of the exotics (and the matter content) in a situation where there is no single distinguished section, or, more precisely, where the torus fibration has two sections 'on equal footing.' The prescription we use gives exactly such a description. The purpose of this paper is to present a model for Heterotic/F-theory duality in which SU(5) symmetry is broken (on both sides) by Wilson lines. The work derives from the previous global $F$-theory model with Wilson line symmetry-breaking [43]. It modifies the previous model so as to allow the construction of a Heterotic dual. It adapts previously known Heterotic techniques for eliminating undesirable features of the model, such as vector-like exotics, by constructing the torus-fibration (on both sides) with two sections [22]. This allows us to use the Heterotic technique of translation by the difference of the two sections to form the requisite $\mathbb{Z}_{2}$-action in order to evade the above-mentioned theorem and eliminate the vector-like exotics. The model has some very nice features. It contains the gauge group $\mathrm{SU}(5)$ broken via a Wilson line in the hypercharge direction to $\mathrm{SU}(3) \times \mathrm{SU}(2) \times \mathrm{U}(1)$. It contains three families of quarks 
and leptons and one pair of Higgs doublets. Furthermore our $F$-theory model exhibits a $\mathbb{Z}_{4} \mathbf{R}$-symmetry in the semi-stable limit of the $F$-theory model.

In addition it has a local $\mathrm{U}(1)_{X}$ gauge symmetry, given by a global $(4+1)$ split spectral cover, where $\mathrm{U}(1)_{X}$ is the $\mathrm{U}(1)$ in $\mathrm{SO}(10)$ commuting with $\mathrm{SU}(5)_{\text {gauge }}$; Higgs pairs have charge, \pm 2 , while SU(5) 10's and $\overline{5}$ 's have charge, +1 and -3 , respectively. This gauge symmetry preserves $R$-parity, eliminating baryon and lepton number violating dimension- 4 operators [24]. $\mathrm{U}(1)_{X}$ can also be used to identify possible right-handed neutrino states which are $\mathrm{SU}(5)$ singlet matter states with $\mathrm{U}(1)_{X}$ charge $+5[9,52]$.

Some problems for this construction are as follows. The gauge symmetry $\mathrm{U}(1)_{X}$ also forbids Majorana masses for the right-handed neutrinos. In addition, in subsection 8.7 we calculate the $D$-term for $\mathrm{U}(1)_{X}$ (see eq. 3.30 in $[24,25]$ ), requiring $D_{\mathrm{U}(1)_{X}}=0$ so that this symmetry is not spontaneously broken by fields derived from the adjoint representation of $E_{8}$. In addition, $\mathrm{U}(1)_{X}$ is not sufficient to prevent dimension-5 baryon and lepton number violating operators. It is possible that the local $\mathrm{U}(1)_{X}$ symmetry is broken down to a global $\mathrm{U}(1)_{X}$ symmetry via a Stueckelberg mechanism [24, 52], but this is beyond the scope of the present paper. The $\mathrm{U}(1)_{X}$ symmetry may also be broken to a $\mathbb{Z}_{2}$ matter parity by a non-perturbative effect at the GUT scale. This would then allow for right-handed neutrino Majorana masses at the GUT scale.

Finally, a very novel feature of the model is that it contains a twin/mirror SU(5) symmetry broken to a mirror SM with three families of mirror quarks and leptons and a pair of mirror Higgs multiplets. ${ }^{1}$ This is a direct consequence of the fact that the GUT surface, $S_{\mathrm{GUT}}^{\vee}=$ Enriques, is a branched (therefore irreducible) double cover of the base $B_{2}$. The gauge and Yukawa couplings in the visible and mirror worlds are determined by volume moduli which must still be stabilized and supersymmetry broken. As a result mirror matter does not necessarily have the same mass as visible matter or the same value of their gauge and Yukawa couplings. This mirror sector is a possible candidate for the dark matter in the universe.

\subsection{The mathematics}

We next give an idea of the mathematics of our model for Heterotic/ $F$-theory duality in which SU(5)-symmetry is broken (on both sides) by a Wilson line construction. As mentioned, the mathematical model derives from a previous global $F$-theory model with Wilson line symmetry-breaking [43]. It modifies the previous model so as to allow the construction of a Heterotic dual. It also adapts previously known Heterotic techniques for eliminating undesirable features of the model, such as vector-like exotics, by constructing the torusfibration (on both sides) that admits two sections. More properly, the two sections, taken together, should be thought of as determining an invariant $\mathbf{g}_{2}^{1}$ linear series on the torus fiber.

\footnotetext{
${ }^{1}$ Mirror world defined as the parity transform of the Standard Model has been reviewed in the paper by L.B. Okun [48]. This paper has many references which we refer to the reader. Some of these references however include another related definition of the mirror world given by a generalized $\mathbb{Z}_{2}$-symmetry which takes the Standard Model into the twin or mirror sector with states having identical charges but different masses and couplings. Papers in this genre include, for example, [7, 13], and [4].
} 
Our goal for this paper is to present a global phenomenonologically consistent Heterotic model $V_{3}^{\vee} / B_{2}^{\vee}$ and $F$-theory dual $W_{4}^{\vee} / B_{3}^{\vee}$. These will be constructed as equivariant $\mathbb{Z}_{2}$-quotients of another set of dual Heterotic/ $F$-theory models $V_{3} / B_{2}$ and $W_{4} / B_{3}$ with respective involutions

$$
\tilde{\beta}_{3} / \beta_{2}: V_{3} / B_{2} \rightarrow V_{3} / B_{2}
$$

and

$$
\tilde{\beta}_{4} / \beta_{3}: W_{4} / B_{3} \rightarrow W_{4} / B_{3}
$$

whose quotients $V_{3}^{\vee} / B_{2}^{\vee}$ and $W_{4}^{\vee} / B_{3}^{\vee}$ are our ultimate goal. However much of the work will center on $V_{3} / B_{2}$ and $W_{4} / B_{3}$ and their respective involutions, passing to the quotients only late in the story. Furthermore much of the work has already been completed in our companion papers $[14,15]$, including in [15] the complete construction of

$$
B_{3}=\mathbb{P}^{1} \times B_{2}
$$

and the involution $\beta_{3}$ and calculation of their numerical invariants. The groundwork contained in those papers will be referred to as needed in what follows.

In short, the fundamental challenge is to construct compatible involutions (1.3) and (1.4) so that both leave their top-degree holomorphic forms invariant, that is, so that their respective quotients are Calabi-Yau manifolds. Because of other necessary characteristics of the Heterotic and $F$-theory models, we showed in [14] that (1.3) must act as

$$
\frac{d x}{y} \mapsto \frac{d x}{y}
$$

on the relative (Weierstrass) one-form on $V_{3} / B_{2}$ whereas (1.4) must act as

$$
\frac{d x}{y} \mapsto-\frac{d x}{y}
$$

on the relative (Weierstrass) one-form on $W_{4} / B_{3}$. We showed this necessity on the $F$-theory side in [14] by tracing the Tate form

$$
w y^{2}=x^{3}+a_{5} w x y+a_{4} z w x^{2}+a_{3} z^{2} w^{2} y+a_{2} z^{3} w^{2} x+a_{0} z^{5} w^{3}
$$

back to its $E_{8}$-origins, namely

$$
w y^{2}=x^{3}+a_{0} z^{5}
$$

We showed in [15] that the coefficients $a_{j}$ of the Tate form, as well as $z$ and $y / x$ must go to minus themselves under the $\mathbb{Z}_{2}$-action. One identifies the configuration of exceptional curves in the crepant resolution of (1.8) with the configuration of the positive simple roots in the $E_{8}$-Dynkin diagram. A consequence of this identification is that (1.6) sends each positive simple root to its negative. We preserve $E_{8}$-symmetry by counteracting this reversal of roots by the operation of complex conjugation on the complex algebraic group $E_{8}^{\mathbb{C}}$, an operation that leaves untouched the compact real form $E_{8}$. This last is reflected in the fact that tracing real roots back to $E_{8}$ requires that (1.7) and (1.8) be rescaled by dividing both sides by $a_{0}^{6}$. This rescales both $z$ and $y / x$ by $a_{0}^{-1}$ and $y / a_{0} x$ indeed becomes invariant under the $\mathbb{Z}_{2}$-action. 


\subsection{The organization of the paper}

In section 2 we derive the Weierstrass equation for the Tate form. We introduce the construction of a second section $\tau$ of $W_{4} / B_{3}$ in addition to the tautological section $\zeta$ 'at infinity.' We discuss the action of the involution $\tilde{\beta}_{4} / \beta_{3}$ as reflecting complex conjugation on the complex algebraic groups $\operatorname{SL}(5, \mathbb{C})$ and $E_{8}^{\mathbb{C}}$ whose compact real forms are $\mathrm{SU}(5)$ and $E_{8}$ respectively.

In section 3 we derive the spectral variety from the Tate form and discuss its decomposition into components of degree four and one respectively.

In sections 4 and 5 we discuss the semi-stable limit and the relation to the Heterotic dual. In particular we show how to build the normal-crossing $K 3$ from an elliptic curve with two flat $E$-bundles.

In section 6 we project the fourfold $W_{4} / B_{3}$ to the $\mathbb{P}^{1}$-bundle $Q / B_{3}$ whose fiber is the degree-2 linear series determined by the two points $\tau\left(b_{3}\right)$ and $\zeta\left(b_{3}\right)$ where the two sections intersect the fiber. We do this by projecting each fiber of $W_{4} / B_{3}$ from the third point of intersection with the line joining $\tau\left(b_{3}\right)$ and $\zeta\left(b_{3}\right)$. This projection, that we name $\bar{W}_{4}$ blows up the singular locus of $W_{4}$. In this way we obtain a commutative diagram

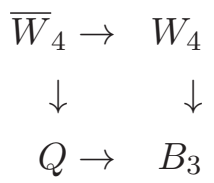

where the top horizontal map is crepant partial resolution, the left-hand vertical map is 2-1 and the bottom horizontal map is a $\mathbb{P}^{1}$-fibration whose fibers correspond to the degree- 2 linear system on the fibers of the right-hand vertical map determined by the lifts of the two sections $(\tau)$ and $(\zeta)$ to $\bar{W}_{4} / B_{3}$. The Calabi-Yau fourfold $\bar{W}_{4}$ is a branched double cover of $Q$. The affine fiber coordinate $\vartheta_{0}$ of $Q / B_{3}$ is a section of $K_{B_{3}}^{-1}$ as are the coefficients $a_{j}$ in the Tate form and $t:=y / x$.

We next construct a full crepant resolution of $\bar{W}_{4}$ to obtain a smooth model $\tilde{W}_{4}$. This is accomplished in section 7. The first step is the partial resolution $W_{4}^{(1)} / B_{3}$ of $\bar{W}_{4} / B_{3}$ that will create an divisor $D_{0}$ connecting the divisors $D_{1}$ (the inherited component from $W_{4} / B_{3}$ ) and $D_{4}$ (the exceptional divisor of $\bar{W}_{4} / W_{4}$ ). Together these three divisors comprise

$$
S_{\mathrm{GUT}} \times_{B_{3}} W_{4}^{(1)} .
$$

This will be followed by a third partial desingularization $W_{4}^{(2)} / B_{3}$ of $W_{4}^{(1)} / B_{3}$ extending over a general point of $S_{\mathrm{GUT}}$. Its exceptional divisor will be reducible so that

$$
S_{\mathrm{GUT}} \times_{B_{3}} W_{4}^{(2)}=D_{0} \cup D_{1} \cup D_{2} \cup D_{3} \cup D_{4}
$$

that configure themselves over a general point of $S_{\mathrm{GUT}}$ as an extended $A_{4}$-Dynkin diagram. That will in turn be followed by a final codimension-2 desingularization $\tilde{W}_{4} / B_{3}$ of $W_{4}^{(2)} / B_{3}$ over the curve $\Sigma_{\overline{5}}^{(44)} \subseteq S_{\mathrm{GUT}}$.

It should be noted that, in the process of putting $(\zeta)$ and $(\tau)$ on equal footing as the first step in the desingularization, neither can be given preference as the one passing 
through the inherited component

$$
S_{\mathrm{GUT}} \times_{B_{3}} \bar{W}_{4} .
$$

The 'inherited' role is assumed by $D_{0}$ while, over a general point of $S_{\mathrm{GUT}}$, the proper transform $(\tilde{\zeta})$ of $(\zeta)$ intersects $D_{1}$ and the proper transform $(\tilde{\tau})$ of $(\tau)$ intersects $D_{4}$.

In section 8 we calculate the Higgs line bundle that will govern the computation of the chiral spectrum. In subsections 8.5 and 8.6 we compute the $G$-flux. In subsection 8.7 we establish the vanishing of the $D$-term for a suitably chosen $\tilde{\beta}$-symmetric Kähler metric.

In section 9 we discuss the symmetry-breaking induced by wrapping the Wilson line on the involution $\tilde{\beta}_{4}$ on $\tilde{W}_{4}$. The Wilson line breaks $\mathrm{SU}(5)$-symmetry to the $\mathrm{SU}(3)_{C} \times$ $\mathrm{SU}(2)_{L} \times \mathrm{U}(1)_{Y}$ symmetry of the Standard Model.

It is in section 10 that we calculate the complete spectrum of the theory. The desired invariants follow rather directly from the results of [15] where the toric presentation of $B_{3}$ and its involution $\beta_{3}$ were explored in detail. The present paper demonstrates a method for eliminating vector-like exotics when breaking the GUT symmetry with the Wilson line. In this section we discuss just one $\mathrm{SU}(5)_{\text {gauge }} \times \mathrm{SU}(5)_{\text {Higgs }}$ sector in the semi-stable limit of the $F$-theory model, however, the massless spectrum in the hidden $\mathrm{SU}(5)_{\text {gauge }} \times \mathrm{SU}(5)_{\text {Higgs }}$ is identical.

Finally in section 11 we discuss the $\mathbb{Z}_{4}$ R-symmetry on the semi-stable limit of our F-theory model.

Remark 1. Throughout this paper, we will employ the following notational convention. Projections with image space $A$ will in general be denoted as $\pi_{A}$. This notation will be employed regardless of the domain of the map, which (hopefully) will be clear from the context.

Remark 2. Throughout this paper, we will let

$$
\mathbb{P}_{\left[i_{1}, \ldots, i_{d}\right]}^{d-1}
$$

denote the weighted complex projective $(d-1)$-space with integer weights $\left[i_{1}, \ldots, i_{d}\right]$ and we will let

$$
\mathbb{P}_{\left[u_{1}, \ldots, u_{d}\right]}
$$

denote the (unweighted) complex projective space with homogeneous coordinates $\left[u_{1}, \ldots, u_{d}\right]$.

\section{$2 W_{4} / B_{3}, E_{8}$-unfolding and its symmetries}

Our starting point is the Fano threefold

$$
B_{3}=\mathbb{P}_{\left[u_{0}, v_{0}\right]} \times B_{2}
$$

where $B_{2}$ is the $D_{2}$ del Pezzo surface studied in section 6 of [15]. $B_{2}$ is a double cover of the projective plane

$$
D_{2} \rightarrow \mathbb{P}_{\left[n_{-1}, m_{i}, m_{-i}\right]}
$$


branched along a specific smooth quartic curve admitting a $\mathbb{Z}_{4}$-action. Denote

$$
N:=c_{1}\left(K_{B_{3}}^{-1}\right)
$$

We form

$$
P:=\mathbb{P}\left(\mathcal{O}_{B_{3}} \oplus \mathcal{O}_{B_{3}}(2 N) \oplus \mathcal{O}_{B_{3}}(3 N)\right)
$$

with homogeneous fiber coordinates $[w, x, y]$ and canonical bundle

$$
\mathcal{O}_{P}(-3) \otimes \pi_{B_{3}}^{*}(-6 N)
$$

For

$$
a_{j}, z, \frac{y}{x}=t \in H^{0}\left(\mathcal{O}_{B_{3}}(N)\right),
$$

we write the Tate form (1.7) for an elliptically fibered fourfold $W_{4} / B_{3}$ in determinantal form as

$$
\left|\begin{array}{cc}
x^{3}+a_{4} z w x^{2}+a_{2} z^{3} w^{2} x+a_{0} z^{5} w^{3} & 1 \\
w y^{2}-\left(a_{5} w x+a_{3} z^{2} w^{2}\right) y & 1
\end{array}\right|=0 .
$$

Since

$$
K_{P}^{-1}=\mathcal{O}_{P}(3(\{w=0\}+6 N)),
$$

$W_{4}$ is Calabi-Yau. Here as in [15] $B_{3}$ admits an involution $\beta_{3}$ with finite fixpoint set with respect to which

$$
t:=\frac{y}{x}, z, a_{0}, a_{2}, a_{3}, a_{4}, a_{5} \in H^{0}\left(\mathcal{O}_{B_{3}}(N)\right)^{[-1]},
$$

the (-1)-eigenspace, and

$$
\begin{aligned}
w & \in H^{0}\left(\mathcal{O}_{P}(1) \otimes \pi_{B_{3}}^{*} \mathcal{O}_{B_{3}}\right)^{[+1]} \\
x & \in H^{0}\left(\mathcal{O}_{P}(1) \otimes \pi_{B_{3}}^{*} \mathcal{O}_{B_{3}}(2 N)\right)^{[+1]} \\
y & \in H^{0}\left(\mathcal{O}_{P}(1) \otimes \pi_{B_{3}}^{*} \mathcal{O}_{B_{3}}(3 N)\right)^{[-1]}
\end{aligned}
$$

with respect to the induced involution on $W_{4} / B_{3}$. The equation $z=0$ defines a smooth surface that, by the adjunction formula, must be a $K 3$-surface.

\subsection{Weierstrass form with respect to $\zeta$}

Let

$$
\zeta: B_{3} \rightarrow W_{4}
$$

be the standard section given by

$$
\zeta\left(b_{3}\right)=\{[w, x, y]=[0,0,1]\}
$$


Referring to (2.4) we change the equation of $W_{4} / B_{3}$ into Weierstrass form based at the section $\zeta$ in the standard way. Namely we complete the square with respect to $y$ as follows.

$$
\begin{aligned}
w y^{2}= & x^{3}+a_{5} w x y+a_{4} z w x^{2}+a_{3} z^{2} w^{2} y+a_{2} z^{3} w^{2} x+a_{0} z^{5} w^{3} \\
& w\left(y^{2}-\left(a_{5} x+a_{3} z^{2} w\right) y+\frac{\left(a_{5} x+a_{3} z^{2} w\right)^{2}}{4}\right)= \\
& x^{3}+a_{4} z w x^{2}+a_{2} z^{3} w^{2} x+a_{0} z^{5} w^{3}+w \frac{\left(a_{5} x+a_{3} z^{2} w\right)^{2}}{4} \\
& w\left(y-\frac{a_{5} x+a_{3} z^{2} w}{2}\right)^{2}=x^{3}+\left(a_{4} z+\frac{a_{5}^{2}}{4}\right) w x^{2}+z^{2}\left(a_{2} z+\frac{a_{3} a_{5}}{2}\right) w^{2} x+z^{4}\left(a_{0} z+\frac{a_{3}^{2}}{4}\right) w^{3} \\
= & x^{3}+A w x^{2}+B z^{2} w^{2} x+C z^{4} w^{3}
\end{aligned}
$$

where

$$
\begin{aligned}
& A=a_{4} z+\frac{a_{5}^{2}}{4} \\
& B=a_{2} z+\frac{a_{3} a_{5}}{2} \\
& C=a_{0} z+\frac{a_{3}^{2}}{4} .
\end{aligned}
$$

Then we eliminate the $x^{2}$-term by setting

$$
\begin{aligned}
& \underline{x}=x+\frac{a_{5}^{2}+4 a_{4} z}{12} w=x+\frac{A}{3} w \\
& \underline{y}=y-\frac{a_{5} x+a_{3} z^{2} w}{2}
\end{aligned}
$$

finally yielding the Weierstass form

$$
w \underline{y}^{2}=\underline{x}^{3}+\left(B z^{2}-\frac{A^{2}}{3}\right) w^{2} \underline{x}+\left(C z^{4}-\frac{A B}{3} z^{2}+\frac{2 A^{3}}{27}\right) w^{3} .
$$

for our Calabi-Yau fourfold $W_{4}$.

The discriminant of (2.6) is given by

$$
\begin{gathered}
4\left(B z^{2}-\frac{A^{2}}{3}\right)^{3}+27\left(C z^{4}-\frac{A B}{3} z^{2}+\frac{2 A^{3}}{27}\right)^{2}= \\
A^{2}\left(4 A C-B^{2}\right) z^{4}+2 B\left(2 B^{2}-9 A C\right) z^{6}+27 C^{2} z^{8} .
\end{gathered}
$$

Expanding the discriminant in powers of $z$ the coefficient of $z^{4}$ becomes $\left(\frac{a_{5}^{2}}{4}\right)^{2}\left(\frac{a_{3}^{2} a_{5}^{2}}{4}-\right.$ $\left.\left(\frac{a_{3} a_{5}}{2}\right)^{2}\right)=0$ and the coefficient of $z^{5}$ does not in general equal zero so that indeed, by Kodaira's classification, we have $A_{4}$-singularities over $S_{\mathrm{GUT}}=\{z=0\}$.

\subsection{The second section}

Beside the standard section $\zeta: B_{3} \rightarrow W_{4}$ we require a second section

$$
\tau: B_{3} \rightarrow W_{4}
$$


defined by

$$
b_{3} \mapsto[w, x, y]=\left[1, z^{2}, z^{3}\right] .
$$

Substituting this section of $P / B_{3}$ into Tate form $(1.7)$, as in $[14,15]$ one concludes that the condition that it lies in $W_{4}$ is

$$
a_{5}+a_{4}+a_{3}+a_{2}+a_{0}=0 .
$$

This section allows a change of the group structure on the fibers of $W_{4} / B_{3}$ by a translation. Intertwining this translation with the action of $\tilde{\beta}_{4}$ allows us both to eliminate vector-like exotics from the $F$-theory model $W_{4}^{\vee} / B_{3}^{\vee}$ and to introduce a $(4+1)$-split in the spectral divisor giving the $\mathrm{U}(1)_{X}$ discussed in the Introduction. Translation of fibers by this section of course leaves the Weierstrass form on smooth elliptic fibers and $I_{1}$-fibers invariant, that is, all fibers over $\left(B_{3}-S_{\mathrm{GUT}}\right)$.

Notice that, over a general point $b_{3} \in B_{3}, \tau-\zeta$ is not of finite order on $\mathrm{Pic}^{0}$ of the cuspidal curve $w y^{2}=x^{3}$ since the parameter $t=\frac{y}{x}=z$ takes all values. So $\tau-\zeta$ is not of finite order in $\mathrm{Pic}^{0}\left(\pi^{-1}\left(b_{3}\right)\right)$ if the $a_{j}$ are sufficiently small. Furthermore, if $a_{5}=-a_{0}$ are small and $a_{2}=a_{3}=a_{4}=0$, the same argument gives

Lemma 1. For a crepant resolution $\tilde{W}_{4} / B_{3}$ of $W_{4} / B_{3}, \tau-\zeta$ is not of finite order in $\operatorname{Pic}^{0}\left(\tilde{W}_{4} / B_{3}\right)$ over a general point of $S_{\mathrm{GUT}}:=\{z=0\} \subseteq B_{3}$.

So these same assertions hold for a general allowable choice of the coefficients $a_{j}$. This fact is essential to the proof in [15] of lemma 6ii) below. It says that our $F$-theory model has a single Higgs doublet.

Over $b_{3} \in B_{3}$ the line in $\pi^{-1}\left(b_{3}\right)$ between $\zeta\left(b_{3}\right)$ and $\tau\left(b_{3}\right)$ is given by the equation $x-z^{2} w=0$. Letting $a_{j k l}:=a_{j}+a_{k}+a_{l}$ and using that $a_{420}=-a_{53}$ we obtain by (2.7) that the third point of intersection with $W_{4}$ is given by substituting $x=z^{2} w$ in (2.4) to obtain

that is,

$$
w\left|\begin{array}{cc}
z^{6} w^{2}+a_{420} z^{5} w^{2} & 1 \\
y^{2}+a_{420} z^{2} w y & 1
\end{array}\right|=0,
$$

$$
y^{2}+a_{420} z^{2} w y-z^{5} w^{2}\left(z+a_{420}\right)=\left(y-z^{3} w\right)\left(y+\left(z+a_{420}\right) z^{2} w\right)=0 .
$$

We denote the third section as

$$
v\left(b_{3}\right)=\left[w, z^{2} w,\left(-z-a_{420}\right) z^{2} w\right] .
$$

Finally, we therefore have a fourth section

$$
\mu: B_{3} \rightarrow W_{4}
$$

defined by the third point of intersection of the tangent line to

$$
\left|\begin{array}{cc}
x^{3}+a_{4} z w x^{2}+a_{2} z^{3} w^{2} x+a_{0} z^{5} w^{3} & 1 \\
w y^{2}-\left(a_{5} w x+a_{3} z^{2} w^{2}\right) y & 1
\end{array}\right|=0
$$

at $v\left(b_{3}\right)$.

Taken together these calculations yield the following. 
Lemma 2. The sections $\zeta, \tau, v$ and $\mu$ of $W_{4} / B_{3}$ satisfy the following relations in $\operatorname{Pic}^{3}\left(W_{4} / B_{3}\right)$ :

$$
\begin{aligned}
3 \cdot \zeta & \in\left|\mathcal{O}_{W_{4} / B_{3}}(1)\right| \\
\zeta+\tau+v & \in\left|\mathcal{O}_{W_{4} / B_{3}}(1)\right| \\
2 v+\mu & \in\left|\mathcal{O}_{W_{4} / B_{3}}(1)\right| .
\end{aligned}
$$

The last two relations imply that

$$
\zeta+\tau \equiv v+\mu,
$$

that is in classical language, the two divisors are members of the same distinguished $g_{2}^{1}$ on $W_{4} / B_{3}$.

\subsection{The quotient Calabi-Yau manifolds}

In order to wrap a Wilson line, we must require that involution $\tilde{\beta}_{4} / \beta_{3}$ in (1.4) with quotient $W_{4}^{\vee} / B_{3}^{\vee}$ be such that $\beta_{3}$ acts fixpoint-freely on the smooth anti-canonical divisor $S_{\mathrm{GUT}} \subseteq$ $B_{3}$ yielding an Enriques surface

$$
S_{\mathrm{GUT}}^{\vee} \subseteq B_{3}^{\vee}
$$

and that the induced involution $\tilde{\beta}_{3} / \beta_{2}$ on the dual Heterotic with quotient $V_{3}^{\vee} / B_{2}^{\vee}$ be such that $\tilde{\beta}_{3}$ acts freely. In order that $W_{4}^{\vee}$ be Calabi-Yau, the involution $\beta_{3}$ on $B_{3}=\mathbb{P}_{\left[u_{0}, v_{0}\right]} \times B_{2}$ must have only finite fixpoint-set since $S_{\mathrm{GUT}}$ is an ample divisor in the Fano manifold $B_{3}$. This forces $\beta_{3}$ to act as $(-1)$ on the meromorphic two-form on $B_{3}$ with pole on $S_{\mathrm{GUT}}$. That in turn forces $\tilde{\beta}_{4}$ with quotient $W_{4}^{\vee} / B_{3}^{\vee}$ to act as $(-1)$ on the relative one-form $d x / y$ on $W_{4} / B_{3}$ since otherwise $W_{4}^{\vee}$ would not be Calabi-Yau. On the other hand, the induced involution $\tilde{\beta}_{3} / \beta_{2}$ on the Heterotic threefold $V_{3} / B_{2}$ must act as $(+1)$ on the relative oneform $d x / y$ since otherwise $V_{3}^{\vee}$ would not be Calabi-Yau. The possibility, even necessity, of the sign-reversal is explained in [14].

\subsection{Three Calabi-Yau fourfolds related by quotienting}

Following [15] we use root systems and toric geometry to actually define three base threefolds and associated Calabi-Yau fourfolds that we denote by

$$
\begin{aligned}
& W_{4}^{\wedge} \rightarrow B_{3}^{\wedge} \\
& W_{4} \longrightarrow B_{3}=\mathbb{P}_{\left[u_{0}, v_{0}\right]} \times B_{2} \\
& W_{4}^{\vee} \longrightarrow B_{3}^{\vee}:=B_{3} /\left\{C_{u, v}\right\} .
\end{aligned}
$$

$B_{3}^{\wedge}$ is defined to be the resolution of the graph of the Cremona involution on $\mathbb{P}\left(\mathfrak{h}_{\mathrm{SU}(5)}\right)$ with respect to a basis given by the choice of a system of simple roots of $\mathrm{SU}(5)$ balanced between positive and negative Weyl chambers. We have reserved the least cumbersome notation for the intermediate one $B_{3}$ because, as we have already mentioned, it is computationally most convenient to work in that setting.

On the $F$-theory side our ultimate target is the (orbifold) Calabi-Yau fourfold $W_{4}^{\vee}$ with smooth Heterotic dual Calabi-Yau threefold $V_{3}^{\vee}$ having two bundles with Yang-Mills connections with structure group symmetry-breaking

$$
E_{8} \stackrel{\text { Tate }}{\Longrightarrow} \mathrm{SU}(5)_{\text {gauge }} \times \mathrm{SU}(5)_{\text {Higgs }} \stackrel{\text { Higgs }}{\Longrightarrow} \mathrm{SU}(5)_{\text {gauge }} \stackrel{\text { Wilson-line }}{\Longrightarrow} \mathrm{SU}(3)_{C} \times \mathrm{SU}(2)_{L} \times \mathrm{U}(1)_{Y},
$$


as described in [15]. Notice that the initial fourfolds and the involutions do not involve any choice of Weyl chamber. It is only the crepant resolutions of the Calabi-Yau fourfolds and threefolds that imply such choices. This is explained in [14].

In order to wrap a Wilson line, we require that $W_{4} / B_{3}$ given in (1.7) admit an equivariant involution $\tilde{\beta}_{4} / \beta_{3}$ with quotient $W_{4}^{\vee} / B_{3}^{\vee}$ such that $\beta_{3}$ acts fixpoint-free on the smooth anti-canonical divisor $S_{\mathrm{GUT}} \subseteq B_{3}$ yielding an Enriques surface

$$
S_{\mathrm{GUT}}^{\vee} \subseteq B_{3}^{\vee}
$$

In order that $W_{4}^{\vee}$ be Calabi-Yau, the involution $\beta_{3}$ on $B_{3}=\mathbb{P}_{\left[u_{0}, v_{0}\right]} \times B_{2}$ must have only finite fixpoint-set since $S_{\mathrm{GUT}}$ is an ample divisor in the Fano manifold $B_{3}$. As explained in [14] this forces $\beta_{3}$ to act as $(-1)$ on the meromorphic two-form on $B_{3}$ with pole on $S_{\mathrm{GUT}}$.

\subsection{Unfolding the $E_{8}$-singularity}

A basic principle in the mathematics of String Theory is that the geometric model (1.7) of $F$-theory must be considered as having evolved according to the unfolding of the $E_{8}$-surface singularity

$$
w y^{2}=x^{3}+a_{0} z^{5} .
$$

In [14] we have observed that principle to the letter, tracing the equivariant crepant resolution implicit in the Tate form (1.7) back to the Brieskorn-Grothendieck equivariant crepant resolution $[11,51]$ of the semi-universal deformation of the rational double point singularity (1.8) by requiring that the section defining $S_{\mathrm{GUT}}$ be given by a formula

$$
z=\sum_{j=2}^{5} \kappa_{j} a_{j}
$$

for generic $\kappa_{j}$.

The assumption (2.7) will, as we will see, reduces the maximal subgroup decomposition

$$
\frac{\mathrm{SU}(5)_{\text {gauge }} \times \mathrm{SU}(5)_{\text {Higgs }}}{\mathbb{Z}_{5}} \subseteq E_{8}
$$

of $[14]$ to

$$
\mathrm{SU}(5)_{\text {gauge }} \times \mathrm{U}(1)_{X} \times \mathrm{SU}(4)_{\text {Higgs }} \subseteq E_{8}
$$

so that, on the $F$-theory side, one begins with the identification of maximal tori compatible with the three-dimensional commutative diagram obtained by pasting the top and bottom morphisms of

$$
\begin{aligned}
& \operatorname{SL}(5 ; \mathbb{C})_{\text {gauge }} \times \operatorname{SL}(4 ; \mathbb{C})_{\text {Higgs }} \times \mathbb{C}^{*} \stackrel{\dot{\kappa}}{\hookrightarrow} \dot{E}_{8}^{\mathbb{C}} \\
& \uparrow \quad \uparrow \\
& \mathrm{SU}(5)_{\text {gauge }} \times \mathrm{SU}(4)_{\mathrm{Higgs}} \times \mathrm{U}(1)_{X} \hookrightarrow E_{8} \\
& \downarrow \downarrow \downarrow \\
& \mathrm{SL}(5 ; \mathbb{C})_{\text {gauge }} \times \mathrm{SL}(4 ; \mathbb{C})_{\text {Higgs }} \times \mathbb{C}^{*} \stackrel{\ddot{\mu}}{\hookrightarrow} \ddot{E}_{8}^{\mathbb{C}}
\end{aligned}
$$


to the top and bottom morphisms, respectively, of the commuting diagram

$$
\begin{aligned}
& \operatorname{SL}(5 ; \mathbb{C})_{\text {gauge }} \times \operatorname{SL}(4 ; \mathbb{C})_{\text {Higgs }} \times \mathbb{C}^{*} \stackrel{\dot{\kappa}}{\hookrightarrow} \dot{E}_{8}^{\mathbb{C}} \\
& \uparrow \iota \uparrow \iota \\
& \mathrm{SL}(5 ; \mathbb{C})_{\text {gauge }} \times \mathrm{SL}(4 ; \mathbb{C})_{\text {Higgs }} \times \mathbb{C}^{*} \stackrel{\ddot{n}}{\hookrightarrow} \ddot{E}_{8}^{\mathbb{C}}
\end{aligned}
$$

of real analytic outer complex conjugation involutions. [14] and [15] are built around the necessity of a choice of a positive-negative pair of Weyl chambers of $E_{8}$ with the requirement that every step of constructions must commute with the passage between these two chambers. In particular, as we have shown in [14], the equivariant crepant resolution $\tilde{W}_{4} / B_{3}$ of $W_{4} / B_{3}$ depends on the choice of Weyl chamber.

This means that we will have two copies of the crepantly resolved $\tilde{W}_{4} / B_{3}$ that we designate by letting $\dot{W}_{4} / B_{3}$ denote the $F$-theory model with a choice of positive chamber and $\ddot{W}_{4} / B_{3}$ with its negative as the choice of positive Weyl chamber. The action of $\tilde{\beta}_{4} / \beta_{3}$ on $\dot{W}_{4} / B_{3}$ will be a holomorphic involution that acts on roots as the longest element of the Weyl group $W \mathrm{SU}(5)$ yielding a Calabi-Yau quotient, and similarly for the quotient of the action on $\ddot{W}_{4} / B_{3}$. However these quotients are only real-analytically equivalent, not complex-analytically equivalent. As explained in [15] the exceptional curves over the quotient $S_{\text {GUT }}^{\vee}$ are 'flopped' when passing from one to the other. The flop is essentially invisible on the Heterotic side since it tracks only the real $E_{8}$-bundles and the 'flop' becomes the passage between the two possible complexifications of the same real $E_{8}$-bundle.

Identifying exceptional components over $S_{\mathrm{GUT}}$ with positive simple roots forces the involution $\tilde{\beta}_{4}$ to act as the non-trivial involution on the $A_{4}$-Dynkin diagram, that is, by the longest element of the Weyl group $W \mathrm{SU}(5)$ on the exceptional divisors of the crepant resolution of $W_{4} / B_{3}$. Again as shown in [14], it is the commutativity of the geometric involutions $\tilde{\beta}_{4} / \beta_{3}$ and $\tilde{\beta}_{3} / \beta_{2}$ with the complex conjugate involution $\iota$ in $(2.10)$ that allows us to incorporate both in the simultaneous quotienting on both the Heterotic and $F$-theory models that preserves initial $E_{8}$-symmetry and subsequent $\mathrm{SU}(5)$-symmetry since (2.10) acts trivially on $\mathrm{SU}(5)$ and $E_{8}$.

\section{The spectral divisor}

The crepant resolution $\tilde{W}_{4} / B_{3}$ of $W_{4} / B_{3}$ will have $I_{5}$-type fibers over generic points of

$$
S_{\mathrm{GUT}}:=\{z=0\} \subseteq B_{3} .
$$

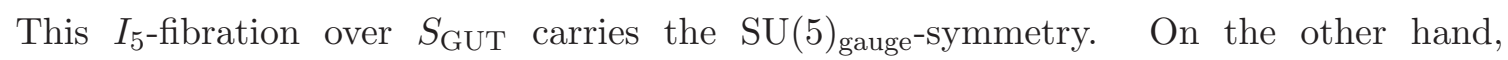
$\mathrm{SU}(5)_{\mathrm{Higgs}^{-}}$-symmetry is broken on a five-sheeted branched covering of $B_{3}$ given by the lift of

$$
\mathcal{C}_{\text {Higgs }}:=W_{4} \cdot\left(\left\{w y^{2}=x^{3}\right\}-\left\{w^{4}=0\right\}\right)
$$

to a divisor $\tilde{\mathcal{C}}_{\text {Higgs }} \subseteq \tilde{W}_{4}$. Its symmetry is broken by assigning non-trivial eigenvalues to the fundamental representation $\mathrm{SU}(5)_{\text {Higgs }}$ using the spectral construction with respect to the push-forward to $B_{3}$ of a line bundle $\mathcal{L}_{\text {Higgs }}$ on $\tilde{\mathcal{C}}_{\text {Higgs }}$. We see this as follows. 
We form

$$
\hat{P}:=\mathbb{P}\left(\mathcal{O}_{B_{3}} \oplus \mathcal{O}_{B_{3}}(N) \oplus \mathcal{O}_{B_{3}}(2 N) \oplus \mathcal{O}_{B_{3}}(3 N)\right)
$$

using the fiber coordinate $t$ for $\mathcal{O}_{B_{3}}(N)$. The natural projection

$$
\hat{P} \rightarrow P
$$

with center $\mathbb{P}\left(\mathcal{O}_{B_{3}}(N)\right)$ is defined except along the section where $x=y=w=0$. $\quad \hat{P}$ contains the smooth five-dimensional incidence hypersurface $Y$ given by the equation

$$
\left|\begin{array}{ll}
x & y \\
w & t
\end{array}\right|=0,
$$

thereby forming a smooth quadric hypersurface over $B_{3}$ with distinguished section given by $x=y=w=0$. So the restriction of (3.2) to $Y$ is defined except along the section where it spreads the exceptional locus over the linear locus $\{x=0\}$ in $P$. The result is a birational morphism

$$
\hat{Y} \rightarrow P
$$

that maps the exceptional locus over the section isomorphically onto $\{x=0\} \subseteq P$ and blows down the linear loci $\{x=y=0\} \subseteq Y$ and $\{x=w=0\} \subseteq Y$.

Ignoring what happens over $\{w=0\}$, that is, setting $w=1$ and using $t$ as the affine fiber we obtain the equation

$$
t^{2} x^{2}=x^{3}+a_{5} x^{2} t+a_{4} z x^{2}+a_{3} z^{2} x t+a_{2} z^{3} x+a_{0} z^{5}
$$

as the defining equation for the hypersurface $\hat{W}_{4}-\{w=0\} \subseteq \hat{Y}-\{w=0\}$. Using

$$
\begin{aligned}
& x=w t^{2} \\
& y=w t^{3}
\end{aligned}
$$

the divisor given by the intersection with $\hat{W}_{4}$ then has equation

$$
a_{5} t^{5}+a_{4} t^{4} z+a_{3} t^{3} z^{2}+a_{2} t^{2} z^{3}+a_{0} z^{5}=0 .
$$

This is the equation on the affine set $w=1$ of the intersection of the proper transform of $W_{4}$ with the locus given by

$$
\left\{y^{2}=x^{3}\right\}
$$

and is called the spectral divisor. The spectral divisor, in particular, contains the singular locus of $W_{4}$. The condition (2.7) implies that homogeneous form in (3.3) is divisible by $z-t$, that is, the spectral divisor admits a $(4+1)$ factorization. The involution $\tilde{\beta}_{4} / \beta_{3}$ takes

$$
(z-t) \mapsto(t-z)
$$

and leaves (3.3) invariant.

Said otherwise, since $W_{4}$ is smooth except over $\{z=0\} \subseteq B_{3}$, the proper transform $\hat{W}_{4} \subseteq \hat{Y}$ of $W_{4}$ blows up the codimension-2 subvariety

$$
\{z=t=0\}
$$


by forming the incidence fivefold

$$
\tilde{Y}=\left\{\left|\begin{array}{ll}
z & t \\
\tilde{z} & \tilde{t}
\end{array}\right|=0\right\} \subseteq Y \times \mathbb{P}_{[\tilde{t}, \tilde{z}]}
$$

with exceptional locus $\mathbb{P}_{[\tilde{t}, \tilde{z}]} \times S_{\mathrm{GUT}}$. The proper transform $\hat{W}_{4}$ of $W_{4}$ intersects this exceptional locus in the hypersurface

$$
\mathcal{D} \subseteq \mathbb{P}_{[\tilde{t}, \tilde{z}]} \times B_{3}
$$

given by the equation

$$
0=a_{5} \tilde{t}^{5}+a_{4} \tilde{z} \tilde{t}^{4}+a_{3} \tilde{z}^{2} \tilde{t}^{3}+a_{2} \tilde{z}^{3} \tilde{t}^{2}+a_{0} \tilde{z}^{5}
$$

(Compare (3.3) with (3.5).)

It is immediate to check that the sections $\zeta$ and $\tau$ both lie in $W_{4} / B_{3}$ and both lie in

$$
S P:=\left\{\left|\begin{array}{cc}
x^{3} & 1 \\
w y^{2} & 1
\end{array}\right|=0\right\} \subseteq P .
$$

For fiber coordinate $t$ for $S P$ with

$$
\begin{aligned}
& w t^{2}:=x \\
& w t^{3}:=y
\end{aligned}
$$

the value $t=z$ gives the section $\tau=\left\{[w, x, y]=\left[1, z^{2}, z^{3}\right]\right\}$ of $W_{4} \cap S P$. The cohomology class of

$$
S P \cap W_{4}
$$

is given by

$$
c_{1}\left(\mathcal{O}_{P}(3)\right)^{2} .
$$

One sees easily that $W_{4}$ and (3.4) have contact of order 4 along $(\zeta)$ and order 1 along $(\tau)$. Given $b_{3} \in\left(B_{3}-S_{\mathrm{GUT}}\right)$, we denote by $S P_{(4)}\left(b_{3}\right)$ the other four points in which $\left.W_{4}\right|_{\pi^{-1}\left(b_{3}\right)}$ intersects $S P$. We denote the closure in $P$ of the locus $\cup_{b_{3} \in\left(B_{3}-S_{\mathrm{GUT}}\right)} S P_{(4)}\left(b_{3}\right)$ as

$$
S P_{(4)} \subseteq S P \cap W_{4}
$$

that since $0=a_{0}+a_{2}+a_{3}+a_{4}+a_{5}$ has equation

$$
\begin{aligned}
a_{5} \tilde{t}^{5}+a_{4} \tilde{t}^{4} \tilde{z}+a_{3} \tilde{t}^{3} \tilde{z}^{2}+a_{2} \tilde{t}^{2} \tilde{z}^{3}+a_{0} \tilde{z}^{5} & = \\
\left(a_{5} \tilde{t}^{4}+a_{54} \tilde{t}^{3} \tilde{z}-a_{20} \tilde{t}^{2} \tilde{z}^{2}-a_{0} z^{3}(\tilde{t}+\tilde{z})\right)(\tilde{t}-\tilde{z}) & =0
\end{aligned}
$$

where $a_{j k}=a_{j}+a_{k}$, etc. ${ }^{2}$

Thus the spectral divisor

$$
\mathcal{C}_{\text {Higgs }}=\mathcal{C}_{\text {Higgs }}^{(4)}+\mathcal{C}_{\text {Higgs }}^{(1)}
$$

\footnotetext{
${ }^{2}$ Often this equation is written in terms of the variable $s=\tilde{t} / \tilde{z}$ so that $(\tilde{t}-\tilde{z})=0$ becomes $s-1=0$, thereby eliminating $\mathbf{1 0}_{\{-4\}}$ representations, as in (70) of [9].
} 
is the image of $\mathcal{D}=\mathcal{D}^{(4)}+\mathcal{D}^{(1)}$ in $\hat{W}_{4} \cdot{ }^{3}$ Thus the involution $\tilde{\beta}_{4} / \beta_{3}$ preserves (3.9). Using that $a_{54320}=0$, the Higgs curve, that will be important throught this paper, is defined as the image in $S_{\mathrm{GUT}}$ of the common solutions to the two equations

$$
\begin{aligned}
a_{5} \tilde{t}^{4}-a_{20} \tilde{t}^{2} \tilde{z}^{2}-a_{0} \tilde{z}^{4}=0 \\
a_{54} \tilde{t}^{2}-a_{0} \tilde{z}^{2}=0 .
\end{aligned}
$$

Writing (3.10) as two equations in the variable $\tilde{t}^{2} / \tilde{z}^{2}$, the solution set doubly covers the surface in $B_{3}$ defined by the resultant equation obtained by substituting

$$
\frac{\tilde{t}^{2}}{\tilde{z}^{2}}=\frac{a_{0}}{a_{54}}
$$

in the first equation to obtain

$$
a_{5}\left(\frac{a_{0}}{a_{54}}\right)^{2}-a_{20}\left(\frac{a_{0}}{a_{54}}\right)-a_{0}=0
$$

that, again using $a_{54320}=0$, reduces to

$$
a_{0} \cdot\left|\begin{array}{cc}
a_{4} & -a_{5} \\
a_{3}+a_{0} & a_{3}
\end{array}\right|=0
$$

with branch locus defined by the restriction of the divisor class $N$.

\subsection{Adjusting the $E_{8}$-evolution}

Because the image $(\tau)$ of our second section $\tau$ actually generates with $\mathcal{C}_{\text {Higgs }}^{(1)}$ in our $F$-theory model $\mathrm{SU}(5)_{\text {gauge }} \times \mathrm{SU}(5)_{\text {Higgs }}$ will be actually replaced by

$$
\mathrm{SU}(5)_{\text {gauge }} \times\left(\mathrm{SU}(4)_{\text {Higgs }} \times \mathrm{U}(1)_{X}\right)
$$

with maximal torus comprising a maximal torus in $E_{8}$ and so a vector space isomorphism of Cartan subalgebras

$$
\mathfrak{h}_{s u(5)} \oplus \mathfrak{h}_{s u(4)} \oplus \mathfrak{h}_{u(1)} \stackrel{\cong}{\rightarrow} \mathfrak{h}_{e_{8}}
$$

and associated commutative diagram

$$
\begin{array}{rr}
\mathrm{SU}(5) \times \mathrm{SU}(4) \times \mathrm{U}(1) & \stackrel{A d_{\mathrm{SU}(5)} \times A d_{\mathrm{SU}(4)} \times A d_{\mathrm{U}(1)}}{\longrightarrow} \mathrm{GL}(s u(5) \times s u(4) \times u(1)) \\
\downarrow & \\
E_{8} \stackrel{A d_{E_{8}}}{\longrightarrow} & \mathrm{GL}\left(e_{8}\right) .
\end{array}
$$

\footnotetext{
${ }^{3}$ By (7.16) below, the $\mathcal{D}^{(1)}$-component projects to the invariant global section $v$ of $W_{4} / B_{3}$ given in lemma 2 , therefore yielding a global $\mathrm{U}(1)_{X}$.
} 
Therefore the restriction of $E_{8} \stackrel{A d_{E_{8}}}{\longrightarrow} \operatorname{Aut}\left(e_{8}\right)$ to $\mathrm{SU}(5)_{\text {gauge }} \times\left(\mathrm{SU}(4)_{\mathrm{Higgs}} \times \mathrm{U}(1)_{X}\right)$ decomposes as in (88) of [9] into

$$
\begin{aligned}
(\mathbf{1 5}, \mathbf{1})_{0} & \oplus \\
(\mathbf{1}, \mathbf{1})_{0} & \oplus(\mathbf{1}, \mathbf{1 0})_{-4} \oplus(\mathbf{1}, \overline{\mathbf{1 0}})_{4} \oplus(\mathbf{1}, \mathbf{2 4})_{0} \\
& \oplus(\mathbf{4}, \mathbf{1})_{5} \oplus(\mathbf{4}, \overline{\mathbf{5}})_{-3} \oplus(\mathbf{4}, \mathbf{1 0})_{1} \\
& \oplus(\overline{\mathbf{4}}, \mathbf{1})_{-5} \oplus(\overline{\mathbf{4}}, 5)_{3} \oplus(\mathbf{4}, \overline{\mathbf{1 0}})_{-1} \\
& \oplus(\mathbf{6}, \mathbf{5})_{-2} \oplus(\mathbf{6}, \overline{\mathbf{5}})_{2} .
\end{aligned}
$$

Here 5 denotes the standard matrix representation

$$
\mathrm{SU}(5) \rightarrow \mathrm{GL}\left(\mathbb{C}^{5}\right)
$$

10 denotes the induced representation

$$
\mathrm{SU}(5) \rightarrow \mathrm{GL}\left(\wedge^{2} \mathbb{C}^{5}\right)
$$

and the 'bar' indicates the conjugate representation obtained by composing with the inverse map on SU(5). Analogously for SU(4).

\section{Semi-stable degeneration and $V_{3} / B_{2}$}

To link a crepant resolution $\tilde{W}_{4} / B_{3}$ to its Heterotic dual $V_{3} / B_{2}$, recall that $B_{3}=B_{2} \times \mathbb{P}_{\left[u_{0}, v_{0}\right]}$ so that one can realize $\tilde{W}_{4} / B_{3}$ as $W_{4,1} / B_{3,1}$ in a family of elliptic Calabi-Yau 4 -folds $W_{4, \delta} / B_{3, \delta}$ constructed over

$$
B_{3, \delta}=B_{2} \times\left\{a^{\prime} b^{\prime}=\delta \cdot a^{\prime \prime} b^{\prime \prime}\right\} \subseteq B_{2} \times \mathbb{P}_{\left[a^{\prime}, a^{\prime \prime}\right]} \times \mathbb{P}_{\left[b^{\prime}, b^{\prime \prime}\right]}
$$

as follows. Using the affine parameters

$$
\begin{aligned}
{\left[a^{\prime}, a^{\prime \prime}\right] } & =[a, 1] \\
{\left[b^{\prime}, b^{\prime \prime}\right] } & =[b, 1]
\end{aligned}
$$

we define the affine family

$$
B_{2} \times\{a b=\delta: 0 \leq \delta \leq 1\} \subseteq B_{2} \times \mathbb{C}^{2}
$$

and identify $\mathbb{P}_{\left[u_{0}, v_{0}\right]}$ with the closure $\left\{a^{\prime} b^{\prime}=a^{\prime \prime} b^{\prime \prime}\right\}$ of $\{a b=1\}$ by the rule

$$
\frac{u_{0}-v_{0}}{u_{0}+v_{0}}=a=b^{-1} \text {. }
$$

That is

$$
\begin{aligned}
& \left|\begin{array}{cc}
u_{0}-v_{0} & u_{0}+v_{0} \\
a^{\prime} & a^{\prime \prime}
\end{array}\right|=0 \\
& \left|\begin{array}{cc}
u_{0}-v_{0} & u_{0}+v_{0} \\
b^{\prime \prime} & b^{\prime}
\end{array}\right|=0 .
\end{aligned}
$$


In this way we identify

$$
\tilde{W}_{4} / B_{3} / B_{2}
$$

as an elliptic fibration over the closure $B_{2} \times\left\{a^{\prime} b^{\prime}=a^{\prime \prime} b^{\prime \prime}\right\}$ of $B_{2} \times\{a b=1\}$. Also as in [15] as coefficients in the Tate form (1.7) for $W_{4} / B_{3}$ we require that

$$
a_{2}, a_{3}, a_{4}, a_{5}, z, t=\frac{y}{x} \in H^{0}\left(K_{B_{3}}^{-1}\right)^{[-1]},
$$

(that is, anti-invariant under the involution $\beta_{3}$ ), in other words they must be linear combinations of the forms listed in table 2 in [15]. Furthermore also as described via table 2 in [15] the four-dimensional family of forms spanned by $a_{2}, a_{3}, a_{4}, a_{5} \in H^{0}\left(K_{B_{3}}^{-1}\right)^{[-1]}$ should deform as

$$
a_{j, \delta}=\delta \cdot a_{j}+a_{j, 0}
$$

where the $a_{j, 0}$ lie in the three-dimensional $(-i)$-eigenspace of the $\mathbb{Z}_{4} \mathbf{R}$-symmetry $T_{u, v}$, again as given in table 2 in [15]. Finally

$$
z_{\delta}:=\delta z+z_{0}
$$

where $z_{0}:=q \cdot\left(u_{0}^{2}-v_{0}^{2}\right)$ is skew-invariant under $\beta_{3}$ so that $q \in H^{0}\left(K_{B_{2}}^{-1}\right)$ must be invariant under the involution

$$
B_{2} \stackrel{\beta_{2}}{\longrightarrow} B_{2}
$$

As we again showed in [15],

$$
B_{2}=D_{2}
$$

is a particular degree-2 del Pezzo surface on which the involution $\beta_{2}$ on $B_{2}$ acts with four fixpoints.

Thus $B_{2, \delta}=B_{2}$ for all $\delta$ and

$$
\begin{aligned}
& B_{3,1}=B_{3} \\
& B_{3,0}=\left(B_{2} \times \mathbb{P}_{\left[a^{\prime}, a^{\prime \prime}\right]}\right) \cup\left(B_{2} \times \mathbb{P}_{\left[b^{\prime}, b^{\prime \prime}\right]}\right)
\end{aligned}
$$

with

$$
\left(B_{2} \times \mathbb{P}_{\left[a^{\prime}, a^{\prime \prime}\right]}\right) \cap\left(B_{2} \times \mathbb{P}_{\left[b^{\prime}, b^{\prime \prime}\right]}\right)=\left(B_{2} \times\{[0,1]\}\right) \cup\left(B_{2} \times\{[0,1]\}\right)
$$

and applying the relations (4.4). $a_{2}, a_{3}, a_{4}, a_{5}$ are required to have no common zeros on $B_{3, \delta}$ for $\delta$ on the half-open real interval $(0,1]$.

The Tate form (1.7) and the action $\beta_{3}$ on $B_{3}$ as described in the tables in section 4 of [15] then determine equivariant involutions $\tilde{\beta}_{4} / \beta_{3}$ on $W_{4} / B_{3}$. For generic choice of $z$, $S_{\mathrm{GUT}}$ will not contain any of the eight fixpoints of $\beta_{3}$ acting on $B_{3}$ so $\beta_{3}$ will act freely on $S_{\mathrm{GUT}}$ yielding a smooth Enriques surface as quotient. The sections $a_{j, \delta}, z_{\delta} \in H^{0}\left(K_{B_{3}}^{-1}\right)$ in the Tate form will be allowed to vary under the contraction (4.6) and in particular the discriminant component $S_{\mathrm{GUT}, \delta} \subseteq B_{3, \delta}$ varies as defined by

$$
z_{\delta}:=\delta \cdot z+(1-\delta) q \cdot\left(u_{0}^{2}-v_{0}^{2}\right)=0
$$

with $\beta_{2}$-invariant $q \in H^{0}\left(K_{B_{2}}^{-1}\right)$. 


\subsection{Degeneration of a single $K 3$-surface}

Over each point $(a, b) \in \mathbb{C}^{2}$ associate the Weierstrass form

$$
y^{2}=x^{3}+g_{2}(a, b) x+g_{3}(a, b)
$$

where $g_{2}$ is homogeneous of total degree 4 and $g_{3}$ homogeneous of total degree 6 . Restrict the Weierstrass form to the locus

$$
\Gamma_{\delta}:=\{(a, b): a \cdot b=\delta\}
$$

so that for $\delta \neq 0$, the discriminant has degree 24. As shown below compactifying at infinity yields a $K 3$-surface elliptically fibered over the closure of $\Gamma_{\delta}$ and setting $\delta=0$ yields the union of two $d P_{9}$ 's meeting over $\{a=b=0\}$. For fixed value $b_{2} \in B_{2}$ the fiber $\pi_{B_{2}}^{-1}\left(b_{2}\right)$ has homogeneous linear coordinates $\left[u_{0}, v_{0}\right]$ and $\pi_{B_{3}}^{-1}\left(\pi_{B_{2}}^{-1}\left(b_{2}\right)\right)$ is a $K 3$-surface in $W_{4}$. Since $g_{2}$ and $g_{3}$ in (4.12) must be homogeneous forms of degree 4 and 6 respectively in $\left[u_{0}, v_{0}\right]$, dividing (4.12) by $\left(u_{0}+v_{0}\right)^{6}$ gives the affine equation

$$
y^{2}=x^{3}+g_{2}(a) x_{0}+g_{3}(a)
$$

where

$$
\begin{aligned}
a & :=\frac{u_{0}-v_{0}}{u_{0}+v_{0}} \\
x & :=\frac{x_{n f}}{\left(u_{0}+v_{0}\right)^{2}} \\
y & :=\frac{y_{n f}}{\left(u_{0}+v_{0}\right)^{3}} .
\end{aligned}
$$

On the $\mathbb{P}^{1}$-fiber over $b_{2} \in B_{2}$ we obtain the Weierstrass form

$$
y^{2}=x^{3}+g_{2}\left(b_{2}, a\right) x+g_{3}\left(b_{2}, a\right)
$$

where $g_{2}\left(b_{2}, a\right)$ is a rational function of $a$ with denominator of degree 4 and $g_{3}\left(b_{2}, a\right)$ can be expressed as a rational function of $a$ with denominator of degree 6 .

Thus one can write a global decomposition of (4.10) with

$$
\begin{aligned}
& g_{2}\left(b_{2}, a\right)=\sum_{j=1}^{4} g_{2, j}^{-}\left(b_{2}\right) a^{-j}+g_{2,0}\left(b_{2}\right)+\sum_{j=1}^{4} g_{2, j}^{+} a^{j} \\
& g_{3}\left(b_{2}, a\right)=\sum_{j=1}^{6} g_{3, j}^{-}\left(b_{2}\right) a^{-j}+g_{3,0}\left(b_{2}\right)+\sum_{j=1}^{6} g_{3, j}^{+} a^{j} .
\end{aligned}
$$

Then, letting $b=a^{-1}$, we can equivalently write

$$
\begin{aligned}
& g_{2}\left(b_{2} ; a, b\right)=\sum_{j=1}^{4} g_{2, j}^{-}\left(b_{2}\right) b^{j}+g_{2,0}\left(b_{2}\right)+\sum_{j=1}^{4} g_{2, j}^{+} a^{j} \\
& g_{3}\left(b_{2} ; a, b\right)=\sum_{j=1}^{6} g_{3, j}^{-}\left(b_{2}\right) b^{j}+g_{3,0}\left(b_{2}\right)+\sum_{j=1}^{6} g_{3, j}^{+} a^{j} .
\end{aligned}
$$

where the $g_{2}$ and $g_{3}$ are the functions on the curve $\{a b=1\}$ over the point $b_{2}$ in $B_{2}$. 
Then for any $b_{2} \in B_{2}$ and any $(a, b) \in \mathbb{C}^{2}$ we can write the Weierstrass form

$$
\begin{aligned}
y^{2}= & \frac{1}{2} x^{3}+\left(\frac{g_{2,0}\left(b_{2}\right)}{2}+\sum_{j=1}^{4} g_{2, j}^{+} a^{j}\right) x+\left(\frac{g_{3,0}\left(b_{2}\right)}{2}+\sum_{j=1}^{6} g_{3, j}^{+} b^{j}\right) \\
& +\frac{1}{2} x^{3}+\left(\frac{g_{2,0}\left(b_{2}\right)}{2} \sum_{j=1}^{4} g_{2, j}^{-}\left(b_{2}\right) b^{j}\right) x+\left(\frac{g_{3,0}\left(b_{2}\right)}{2}+\sum_{j=1}^{6} g_{3, j}^{-}\left(b_{2}\right) b^{j}\right) .
\end{aligned}
$$

\section{$4.2 d P_{9}$-bundles over $B_{2}$ giving a singular Calabi-Yau fourfold}

As in section 4.1 we consider $\left(\mathbb{P}^{1}\right)^{2}$ with coordinates $\left(\left[a^{\prime}, a^{\prime \prime}\right],\left[b^{\prime}, b^{\prime \prime}\right]\right)$ as a fiber of the total space

$$
\mathbb{P}\left(K_{B_{2}}^{-1} \oplus K_{B_{2}}^{-1}\right) \times_{B_{2}} \mathbb{P}\left(K_{B_{2}}^{-1} \oplus K_{B_{2}}^{-1}\right) .
$$

The union of our two $d P_{9}$-bundles is then given as the subspace of defined by (5.11) and the equation

$$
a^{\prime} b^{\prime}=0
$$

Returning to our fibration

$$
\pi_{B_{2}}: B_{3} \rightarrow B_{2}
$$

for each fiber $\pi_{B_{3}}^{-1}\left(b_{3}\right)$ of $\tilde{W}_{4} / B_{3}$ we will associate two copies of the Weierstrass equation, namely the one distinguished by designating $\tilde{\zeta}\left(b_{3}\right)$ as the identity element of the group structure and the other distinguished by designating $\tilde{\tau}\left(b_{3}\right)$ as the identity element of the group structure. The two fibers $\pi_{B_{3}}^{-1}\left(b_{3}\right)$ and $\pi_{B_{3}}^{-1}\left(\beta\left(b_{3}\right)\right)$ are identified under the isomorphism induced by the involution $\tilde{\beta}_{4}$ induced by the involution $\beta_{3}$ on $B_{3}$. However, as will become clear in section 6 the line bundle

$$
\mathcal{O}_{\pi_{B_{3}}^{-1}\left(b_{3}\right)}\left(\tilde{\zeta}\left(b_{3}\right)-\tilde{\tau}\left(b_{3}\right)\right)
$$

is not trivial over any $b_{3} \in S_{\mathrm{GUT}}$. As we have seen in (2.6), the identification acts on sheaves $\mathcal{F}$ on the elliptic curve in Weierstrass form

$$
w y_{n f}^{2}=x_{n f}^{3}+g_{2} w^{2} x_{n f}+w^{3}
$$

where $g_{2}\left(b_{3}\right)=\left(B z^{2}-\frac{A^{2}}{3}\right)$ and $g_{3}\left(b_{3}\right)=\left(C z^{4}-\frac{A B}{3} z^{2}+\frac{2 A^{3}}{27}\right)$. The action on $\mathcal{F}$ is given on $\pi_{B_{3}}^{-1}\left(b_{3}\right)$ by

$$
\mathcal{F} \mapsto \mathcal{F} \otimes \mathcal{O}_{\pi_{B_{3}}^{-1}\left(b_{3}\right)}\left(\tilde{\zeta}\left(b_{3}\right)-\tilde{\tau}\left(b_{3}\right)\right)
$$

so that for each $b_{2} \in B_{2}$ the $K 3$-surface $\left(\pi_{B_{2}} \circ \pi_{B_{3}}\right)^{-1}\left(b_{2}\right)$ can be obtained as the smoothing the union of two $d P_{9}$ 's described in subsubsection 4.1 . That is, we realize $\tilde{W}_{4} / B_{2}$ as the smoothing of two $d P_{9}$-bundles

$$
d P_{a} \cup d P_{b}
$$

over $B_{2}$. 
Simultaneously, via

$$
S_{\mathrm{GUT}, \delta}:=\left\{\delta z+(1-\delta) z_{0}=0\right\} \subseteq B_{2},
$$

we move $S_{\mathrm{GUT}}$ to the reducible quadric $z_{0}$ given by

$$
z_{0}=\left(u_{0}^{2}-v_{0}^{2}\right) \cdot q\left(u_{1}, v_{1}, u_{2}, v_{2}\right)
$$

where $q\left(u_{1}, v_{1}, u_{2}, v_{2}\right)$ is invariant under the action of the involution $\beta_{2}$ on $B_{2}$. So at $\delta=0$ $S_{\mathrm{GUT}}$ splits into two 'horizontal' components given by $u_{0}-v_{0}=0$ and $u_{0}+v_{0}=0$ and a 'vertical' component given by $\pi_{B_{2}}^{-1}\left(\left\{q\left(u_{1}, v_{1}, u_{2}, v_{2}\right)=0\right\}\right)$. Thus the intersection

$$
S_{\mathrm{GUT}, \delta} \cap \pi_{B_{2}}^{-1} \text { (fixpoint set of } \beta_{2} \text { ) }
$$

is independent of $\delta$.

From the Weierstrass forms just above, we read off that $\tilde{W}_{4}$ is the smoothing over $a b=1$ of the union of the two $d P_{9}$-bundles given over each point $b_{2} \in B_{2}$ by

$$
\frac{1}{2} y^{2}=\frac{1}{2} x^{3}+\left(\frac{g_{2,0}\left(b_{2}\right)}{2}+\sum_{j=1}^{8} g_{2, j}^{+} a^{j}\right) x+\left(\frac{g_{3,0}\left(b_{2}\right)}{2}+\sum_{j=1}^{12} g_{3, j}^{+} a^{j}\right)
$$

and

$$
\frac{1}{2} y^{2}=\frac{1}{2} x^{3}+\left(\frac{g_{2,0}\left(b_{2}\right)}{2} \sum_{j=1}^{8} g_{2, j}^{-}\left(b_{2}\right) b^{j}\right) x_{0}+\left(\frac{g_{3,0}\left(b_{2}\right)}{2}+\sum_{j=1}^{12} g_{3, j}^{-}\left(b_{2}\right) b^{j}\right) .
$$

These two $d P_{9}$ 's over $b_{2}$ contain the common fiber of $V_{3} / B_{2}$ whose Weierstass form is

$$
w y^{2}=x^{3}+w^{2} g_{2,0}\left(b_{2}\right) x+w^{3} g_{3,0}\left(b_{2}\right) .
$$

The spectral data on the Heterotic side, namely the two $E_{8}$-bundles on the fiber of $V_{3} / B_{2}$ over $b_{2}$, are given via the Friedman-Morgan-Witten classification [23] by the two $d P_{9}$ bundles. Namely in section 4.2 of [23] Friedman-Morgan-Witten give a classifying space for imbeddings of an elliptic fiber $E_{b_{2}}$ of $V_{3} / B_{2}$ into a rational elliptic surface $d P_{9}\left(b_{2}\right)$, each such corresponding canonically by a theorem of E. Looijenga [41] to an isomorphism class of flat $E_{8}^{\mathbb{C}}$-bundles $F$ over $E_{b_{2}}$. Considered as fibrations over $B_{2}$, fibers are so-called $d P_{9}$-surfaces. Setting $[s, t]=\left[a^{\prime}, a^{\prime \prime}\right]$, respectively $[s, t]=\left[b^{\prime}, b^{\prime \prime}\right]$ for $s, t$ as in [23], fibers are given uniquely in $\mathbb{P}_{1,1,2,3}^{3}$ by an equation

$$
y^{2}=4 x^{3}-\left(g_{2} t^{4}-\beta_{1} s t^{3}-\ldots-\beta_{4} s^{4}\right) x-\left(g_{3} t^{6}-\alpha_{2} s^{2} t^{4}-\ldots-\alpha_{6} s^{6}\right)
$$

of weighted homogeneous degree 6 in the variables $x, y, s, t$ with respective weights $2,3,1,1$ over the weighted projective space with coordinates

$$
\left[\alpha_{2}, \ldots, \alpha_{6}, \beta_{1}, \ldots, \beta_{4}\right] \in \mathbb{P}_{[2,3,4,5,6,1,2,3,4]}^{8}
$$

weighted as per their respective indices. 
So to realize the semi-stable degeneration geometrically, the crepant resolution $\tilde{W}_{4} / B_{3}$ of $W_{4} / B_{3}$ is given over the locus

$$
\Gamma_{1}=\{(a, b): a b=1\} \subseteq B_{3} \times \mathbb{C}^{2} .
$$

The deformation in this section is given by restricting the Weierstrass form to the locus

$$
\Gamma_{\delta}=\{(a, b): a b=\delta\}
$$

as $\delta$ goes to 0 . Furthermore $S_{\mathrm{GUT}}$ deforms with $\delta$ via the formula

$$
z_{\delta}=\delta \cdot z+z_{0}
$$

We thereby obtain the family $\left\{\tilde{W}_{4, \delta}\right\}$ of fourfolds over the affine line $\mathbb{C}_{\delta}^{*}$ as in subsection 4.1.

Then the Heterotic model $\left(V_{3}, F_{a}, F_{b}\right)$ over $B_{2}$ canonically corresponds to a normal crossing Calabi-Yau 4-fourfold with two components $d P_{a}$ and $d P_{b}$ obtained by making the construction described just above equivariantly over $B_{2}$.

Thus the family $W_{4, \delta} / B_{3, \delta}$ defined by the Tate form on $B_{3, \delta}$ degenerates as $\delta$ approaches zero to a reducible Calabi-Yau 4-fold

$$
W_{4,0}=d P_{a} \cup d P_{b}
$$

over

$$
\left(B_{2} \times \mathbb{P}_{[a, 1]}\right) \cup\left(B_{2} \times \mathbb{P}_{[b, 1]}\right)
$$

with

$$
V_{3}=d P_{a} \cap d P_{b}
$$

giving the Heterotic model $V_{3} / B_{2}$ defined by $a=b=0$. Finally there are common distinguished sections on every $W_{4, \delta} / B_{3, \delta}$. defined equivariantly by

$$
\begin{aligned}
\zeta_{\delta} & =\{w=x=0\} \\
\tau_{\delta} & =\left\{[w, x, y]=\left[w, w z_{\delta}^{2}, w z_{\delta}^{3}\right]\right\} .
\end{aligned}
$$

The Calabi-Yau threefold

$$
V_{3}=d P_{a} \cap d P_{b}
$$

is elliptically fibered over $B_{2} \times\{a=b=0\}$, the general fiber of $d P_{a} / B_{2}$ has an $I_{5}$-fiber at $a=\infty$ and the general fiber of $d P_{b} / B_{2}$ has an $A_{4}$-fiber at $b=\infty$.

\subsection{The involution}

Then by construction the involution $\tilde{\beta}_{4} / \beta_{3}$ induces involutions $\tilde{\beta}_{4, \delta} / \beta_{3, \delta}$ on $W_{4, \delta} / B_{3, \delta}$ for all $\delta \geq 0$. Furthermore the above asssumptions force

$$
\begin{array}{rr}
W_{4,0} / B_{3,0} \stackrel{\tilde{\beta}_{4,0}}{\longrightarrow} & W_{4,0} / B_{3,0} \\
\downarrow & \downarrow \\
B_{2} \stackrel{\beta_{2}}{\longrightarrow} & B_{2}
\end{array}
$$


to be given by the elliptic fibrations with involutions

$$
\begin{gathered}
d P_{a} /\left(B_{2} \times \mathbb{P}_{[a, 1]}\right) \stackrel{\tilde{\beta}_{a}}{\longrightarrow} d P_{9, a} /\left(B_{2} \times \mathbb{P}_{[a, 1]}\right) \\
\left([w, x, y],\left(b_{2},[a, 1]\right)\right) \mapsto\left([w, x,-y],\left(\beta\left(b_{2}\right),[-a, 1]\right)\right)
\end{gathered}
$$

and

$$
\begin{aligned}
d P_{9, b} /\left(B_{2} \times \mathbb{P}_{[b, 1]}\right) \stackrel{\tilde{\beta}_{b}}{\longrightarrow} d P_{9, b} /\left(B_{2} \times \mathbb{P}_{[b, 1]}\right) \\
\left([w, x, y],\left(b_{2},[b, 1]\right)\right) \mapsto\left([w, x,-y],\left(\beta\left(b_{2}\right),[-b, 1]\right)\right) .
\end{aligned}
$$

The action of these involutions over the fixpoints of the action of $\beta_{2}$ on $B_{2}$ is treated in detail in section 2.4 of [14].

\section{Passing from Heterotic theory to F-theory}

Essentially one passes from the Heterotic model to the $F$-theory model by reading the subsections of the previous section in reverse order and from bottom to top. We can paste one of these $d P_{9}$ along $E$ at $s=0$ and the other along $E$ at $s=0$ to obtain a normal crossing elliptic $K 3$-surface with unobstructed deformation space thereby joining $d P_{a}^{\vee}$ to $d P_{b}^{\vee}$ to form a normal crossing Calabi-Yau fourfold. Having that, a theorem of Kawamata-Namikawa [31] guarantees that the normal crossing elliptic Calabi-Yau fourfold has an unobstructed deformation theory. $B_{3}=\mathbb{P}_{\left[u_{0}, v_{0}\right]} \times B_{2}$ so that our only choices are the section of $K_{B_{2}}^{-1}$ in the definition of $z_{0}$ and the smooth section $z$ of $K_{B_{3}}^{-1}$ in (4.19).

\subsection{Initial data}

In Heterotic theory, we begin with the smooth degree-2 del Pezzo del Pezzo $\left(B_{2}, \beta_{2}\right)$ with involution $\beta_{2}$ with 4 fixpoints constructed in [15]. Letting $B_{2}^{\vee}$ denote the quotient, we are given a smooth, elliptically fibered Calabi-Yau threefold

$$
V_{3}^{\vee} \rightarrow B_{2}^{\vee}
$$

with Weierstrass form

$$
y^{2}=x^{3}+g_{2}\left(b_{2}\right) x+g_{3}\left(b_{2}\right)
$$

for each $b_{2} \in B_{2}^{\vee}$. This Weierstrass equation lives at each point $b_{2}$ of a $\mathbb{P}_{[w, x, y]}$-bundle over $B_{2}$ with

$$
\begin{array}{r}
x \in H^{0}\left(K_{B_{2}}^{-2}\right) \\
y \in H^{0}\left(K_{B_{2}}^{-3}\right) \\
g_{2} \in H^{0}\left(K_{B_{2}}^{-4}\right) \\
g_{3} \in H^{0}\left(K_{B_{2}}^{-6}\right)
\end{array}
$$

in order that $V_{3}^{\vee}$ have trivial canonical bundle. Has fundamental group $\mathbb{Z}_{2}$. 
Furthermore $V_{3}^{\vee}$ supports two principal $E_{8}$-bundles

$$
F_{a}^{\vee} \oplus F_{b}^{\vee}
$$

with Yang-Mills connections. Pulling back via

$$
\begin{aligned}
V_{3}=B_{2} \times_{B_{2}^{\vee}} V_{3}^{\vee} & \rightarrow B_{2} \\
\downarrow & \downarrow \\
V_{3}^{\vee} & \rightarrow B_{2}^{\vee}
\end{aligned}
$$

we have two $\beta_{2}$-invariant $E_{8}$-bundles, $F_{a}$ and $F_{b}$ each with a Yang-Mills connection with fiber over $b_{2} \in B_{2}$.

The smooth, torus-fibered Calabi-Yau threefold $V_{3}^{\vee} / B_{2}^{\vee}$ with fundamental group $\mathbb{Z}_{2}$ is also endowed with two disjoint sections

$$
\zeta, \tau: B_{2}^{\vee} \rightarrow V_{3}^{\vee}
$$

The pull-back of the two sections under

$$
\pi_{B_{2}^{\vee}}: B_{2} \rightarrow B_{2}^{\vee}
$$

becomes the union of two disjoint sections

$$
\zeta, \tau: B_{2} \rightarrow V_{3}:=B_{2} \times_{B_{2}^{\vee}} V_{3}^{\vee} .
$$

Also, following section 2.4 of [14], the fiber of the smooth threefold $V_{3}^{\vee} / B_{2}^{\vee}$ over the four orbifold points of $B_{2}^{\vee}$ must be acquired with multiplicity two, being doubly covered by the elliptic fibers $E_{b_{2}}$ of $V_{3} / B_{2}$ over the $\beta_{2}$-fixpoints $b_{2} \in B_{2}$. The covering must be unbranched via translation by a distinguished half-period. Given the prior conditions imposed on our model that half-period must be $\mathcal{O}_{E_{b_{2}}}\left(\zeta\left(b_{2}\right)-\tau\left(b_{2}\right)\right)$. The role of the logarithmic transform over a neighborhood of each $\beta_{2}$-fixpoint, especially how it induces the action

$$
\left(b_{2},(x, y)\right) \mapsto\left(\beta_{2}\left(b_{2}\right),(x, y)\right)
$$

of $\tilde{\beta}_{3} / \beta_{2}$ on (Weierstrass) fibers of $V_{3} / B_{2}$, as well as the transition from the $\beta_{2}$-pull-backs

$$
F_{a} \oplus F_{b}
$$

is explained in [14].

The Yang-Mills connections on the bundles (5.3) restrict to sums of eight flat line bundles on each fiber of $V_{3} / B_{2}$ and are completely determined by that family of restrictions.

\subsection{Building a normal-crossing $K 3$ from an elliptic curve with two flat $E_{8^{-}}$ bundles}

As in (4.18) the two flat $E_{8}$-bundles on the elliptic fiber $E_{b_{2}}$ of $V_{3} / B_{2}$ over $b_{2}$ determine two $d P_{9}$-bundles

$$
d P_{a}\left(b_{2}\right) \cup d P_{b}\left(b_{2}\right)
$$


with $E_{b_{2}}$ given in each by

$$
s=0 .
$$

That is, over $B_{2}$ we have the union

$$
d P_{a} \cup d P_{b}
$$

of two $d P_{9}$-bundles with

$$
d P_{a} \cap d P_{b}=V_{3} .
$$

Since the canonical bundle of this normal-crossing variety is trivial, the theorem of Kawamata-Namikawa cited above establishes that its deformation space is unobstructed. In fact we employ the very specific smoothing determined by (4.2) and (4.6).

Finally for the homogeneous coordinates $\left[u_{0}, v_{0}\right]$ in $[15]$ and the curve (4.3), the equations

$$
\begin{aligned}
& \left|\begin{array}{cc}
u_{0}-v_{0} & u_{0}+v_{0} \\
a^{\prime} & a^{\prime \prime}
\end{array}\right|=0 \\
& \left|\begin{array}{cc}
u_{0}+v_{0} & u_{0}-v_{0} \\
b^{\prime} & b^{\prime \prime}
\end{array}\right|=0
\end{aligned}
$$

allow us as in (4.2) to form the family of hypersurfaces

$$
B_{3, \delta} \subseteq B_{2} \times\left(\mathbb{P}_{\left[a^{\prime}, a^{\prime \prime}\right]} \times \mathbb{P}_{\left[b^{\prime}, b^{\prime \prime}\right]}^{1}\right) \rightarrow B_{2}
$$

with fibers given by

$$
\left\{a^{\prime} \cdot b^{\prime}=\delta \cdot a^{\prime \prime} \cdot b^{\prime \prime}\right\}
$$

with

$$
K_{B_{3, \delta}}^{-1}=\left.K_{B_{2}}^{-1} \otimes\left(\mathcal{O}_{\mathbb{P}^{1}}(1) \otimes \mathcal{O}_{\mathbb{P}^{1}}(1)\right)\right|_{\left\{a^{\prime} \cdot b^{\prime}=\delta \cdot a^{\prime \prime} \cdot b^{\prime \prime}\right\}}=: N_{\delta} .
$$

The forms (4.6) then yield our $F$-theory model $W_{4} / B_{3}$ at $\delta=1$.

\subsection{The action of the involution on the Heterotic model}

In section 4.2 of [23] Friedman-Morgan-Witten gives a classifying space for imbeddings of an elliptic curve $E$ in Weierstrass form into $d P_{9}$ 's, each corresponding canonically to an isomorphism class of flat $E_{8}$-bundles

$$
F \rightarrow E \text {. }
$$

The flat $E_{8}$-bundle $F$ is given as the sum of eight flat line bundles, each given by the divisor of the form

$$
p-e
$$

where $e=(\tilde{\zeta}) \cap E$ is the identity element of $E$ considered as an abelian group and $p$ is a geometrically given point on the torus $E$. Again we follow Friedman-Morgan-Witten in [14] and consider the family of $d P_{9}$-hypersurfaces

$$
y^{2}=4 x^{3}-\left(g_{2} t^{4}-\beta_{1} s t^{3}-\ldots-\beta_{4} s^{4}\right) x-\left(g_{3} t^{6}-\alpha_{2} s^{2} t^{4}-\ldots-\alpha_{6} s^{6}\right)
$$


in $\mathbb{P}_{1,1,2,3}^{3}$ where the $\alpha_{j}$ and $\beta_{j}$ are homogeneous forms of weight $j$ in a $\mathbb{P}_{1,2,2,3,3,4,4,5,6}^{8}$. Fixing the values of $\alpha_{j}$ and $\beta_{j}$ we think of the solution set of (5.5) as a rational hypersurface in $\mathbb{P}_{1,1,2,3}^{3}$ with distinguished pencil

$$
\gamma s+\delta t=0 .
$$

The given elliptic curve $E$ sits in each $d P_{9}$ in (5.5) as the solution set to the equation

$$
s=0 .
$$

If we change the basepoint of the elliptic curve $E_{b_{3}}$ from $\tilde{\zeta}\left(b_{3}\right)$ to $\tilde{\tau}\left(b_{3}\right)$, then the trivial bundle goes to itself so that translation by $\left(\tilde{\tau}\left(b_{3}\right)-\tilde{\zeta}\left(b_{3}\right)\right)$ acts trivially on $\operatorname{Pic}^{0}\left(E_{b_{3}}\right)$ and therefore acts trivially on flat $E_{8}$-bundles on $E_{b_{3}}$. However changing the identity element on the torus $E$ from $(\tilde{\zeta}) \cap E$ to $(\tilde{\tau}) \cap E$ and then applying the Friedman-Morgan-Witten dictionary gives a different sum of eight flat line bundles, namely those obtained by divisors

$$
p-e+((\tilde{\zeta}) \cap E)-((\tilde{\tau}) \cap E)
$$

that is, all eight flat line bundles are tensored with the non-trivial flat line bundle

$$
\mathcal{O}_{\tilde{W}_{4}}((\tilde{\zeta})-(\tilde{\tau}))
$$

\section{Lemma 3.}

i) The induced action of the involution $\tilde{\beta}_{4,0}$ on the union of the two $d P_{9}$-bundles at $\delta=z_{0}=0$ takes each of the two to itself.

ii) The action of the involution $\tilde{\beta}_{3}$ on the intersection $V_{3}$ of the two $d P_{9}$-bundles is given by the map

$$
\left(b_{2} ; x, y\right) \mapsto\left(\beta_{2}\left(b_{2}\right) ; x, y\right)+\left(\tilde{\zeta}\left(\beta_{2}\left(b_{2}\right)\right)-\tilde{\tau}\left(\beta_{2}\left(b_{2}\right)\right)\right)
$$

where addition is with respect to the addition law on the elliptic curve and the (identical) Weierstass forms

$$
\begin{aligned}
& y^{2}=x^{3}+g_{2}\left(b_{2}\right) x+g_{3}\left(b_{2}\right) \\
& y^{2}=x^{3}+g_{2}\left(\beta\left(b_{2}\right)\right) x+g_{3}\left(\beta\left(b_{2}\right)\right) .
\end{aligned}
$$

iii) Over fixpoints $b_{2}$ of the action of $\beta_{2}$ on $B_{2}, \tau\left(b_{2}\right)-\zeta\left(b_{2}\right)$ is a non-trivial half-period on the fiber $E_{b_{2}}$ of $V_{3} / B_{2}$ but the action of $\tilde{\beta}_{3}$ on the intersection cycle

$$
4 \cdot \zeta\left(b_{2}\right)+5 \cdot \tau\left(b_{2}\right)
$$

of length nine determining the $E_{8}$-bundles on the fiber is given by tensoring with the canonical section of the line bundle associated to the divisor $\zeta\left(b_{2}\right)-\tau\left(b_{2}\right)$.

Proof.

i) This assertion is immediate from the definition of $\tilde{\beta}_{4}$. 
ii) First of all the involution is given by

$$
\begin{aligned}
(x, y) & \mapsto(x,-y) \\
a & \mapsto-a
\end{aligned}
$$

but the fiber of

$$
V_{3} / B_{2}
$$

is given in (4.16) and (4.17) as

$$
y^{2}=x^{3}+g_{2}^{0} x+g_{3}^{0}
$$

so that it does not reflect the sign change of $a$ and we showed in [14] how the sign change of $y$ is absorbed in the process of taking residue. Secondly the equations of the two $K 3$-surfaces fibers

$$
\tilde{W}_{4} \times_{B_{2}}\left\{b_{2}\right\} \cup \tilde{W}_{4} \times_{B_{2}}\left\{\beta\left(b_{2}\right)\right\}
$$

are invariant under $\tilde{\beta}_{4}$ and so are identical. Furthermore by (5.8) the globally defined relative holomorphic 2-form on $\tilde{W}_{4} / B_{2}$ is invariant under the action of $\tilde{\beta}_{4}$. Thus, for any point $b_{3} \in B_{3}$ lying over $b_{2} \in B_{2}$, the relative one-form on $\tilde{W}_{4} / B_{2}$ obtained from the contraction of the relative holomorphic 2 -form on $\tilde{W}_{4} / B_{2}$ against a section of the relative tangent bundle of $B_{3} / B_{2}$ is invariant under the action of $\tilde{\beta}_{4}$. So the induced relative one-form on $V_{3} / B_{2}$ is invariant under the action of $\tilde{\beta}_{3}$. So as we showed in [14] $\tilde{\beta}_{3}$ must act as a translation of distinguished basepoint with respect to the common Weierstrass form (5.7). Since

$$
\begin{aligned}
\tilde{\zeta}\left(b_{2}\right) & \mapsto \tilde{\tau}\left(b_{2}\right) \\
\tilde{\zeta}\left(\beta\left(b_{2}\right)\right) & \mapsto \tilde{\tau}\left(\beta\left(b_{2}\right)\right)
\end{aligned}
$$

respectively, ii) is proved.

iii) Choosing $a_{5} \neq 0$ at the fixpoints $b_{3}$ of the action of $\tilde{\beta}$ on $B_{3}$ separates $\zeta\left(b_{3}\right)$ from $\tau\left(b_{3}\right)$. The assertion is forced by the fact that, in the canonical identification of an elliptic curve $E$ with $\operatorname{Pic}^{0}(E)$, the trivial bundle is associated to $\zeta\left(b_{3}\right)$ for the Weierstrass form based at $\zeta\left(b_{3}\right)$ and is associated to $\tau\left(b_{2}\right)$ for the Weierstrass form based at $\tau\left(b_{3}\right)$.

Said otherwise the involution on $d P_{9}$-structures (5.5) induced by

$$
\begin{aligned}
y & \mapsto-y \\
(s, t) & \mapsto(-s, t)
\end{aligned}
$$

induces the involution

$$
\begin{aligned}
\operatorname{Pic}^{0}(E) & \rightarrow \operatorname{Pic}^{0}(E) \\
L & \mapsto L^{-1}
\end{aligned}
$$


so that there is a unique translation

$$
\begin{aligned}
E & \rightarrow E \\
x & \mapsto x+\left(e^{\prime}-e\right)
\end{aligned}
$$

such that the composition takes the trivial bundle to itself under the change of basepoint of the torus $E$ from $e$ to $e^{\prime}$. Using the decomposition

$$
\operatorname{Pic}^{0}(E)^{\oplus 8}
$$

of the moduli space of semi-stable $E_{8}$-bundles on $E$, the functorial diagram

$$
\begin{array}{rr}
F_{a} \stackrel{\tilde{\beta}_{3}^{*}}{\longrightarrow} & F_{a} \\
\downarrow & \downarrow \\
\operatorname{Pic}^{0}\left(V_{3} / B_{2}\right)^{\oplus 8} \stackrel{\left(\otimes \mathcal{O}_{\tilde{W}_{0}}(\tilde{\zeta}-\tilde{\tau})\right) \circ()^{-1}}{\longrightarrow} \operatorname{Pic}^{0}\left(V_{3} / B_{2}\right)^{\oplus 8}
\end{array}
$$

is such that the bottom horizonal map is the identity map. This is what allows us to induce a semi-stable $E_{8}$-bundle on the quotient

$$
B_{2}^{\vee}=\frac{B_{2}}{\left\{\beta_{2}\right\}} .
$$

See [14] for a more detailed analysis.

Remark 3 . The classifying space for flat $E_{8}$-structures on the elliptic curve $E$ is acted on by the semi-direct product

$$
\operatorname{Pic}^{0}(E) \rtimes \mathbb{Z}_{2}
$$

where $\mathbb{Z}_{2}$ is generated by

$$
([s, t],(x, y)) \mapsto([-s, t],(x,-y)) .
$$

\section{$5.4 \mathrm{SU}(5)_{\text {gauge }}$-roots and the semi-stable limit}

The action of $\tilde{\beta}_{3}$ on

$$
V_{3} / B_{2}=d P_{a} \cap d P_{b}
$$

is explained in [14] through the lens of its local action over a neighborhood of $\beta_{2}$-fixpoints, as is the compatibility of this action with the action induced by $\tilde{\beta}_{4}$ on the semi-stable degeneration

$$
\tilde{W}_{4} / B_{2} \Rightarrow d P_{a} \cup d P_{b} .
$$

One checks directly that this action is compatible with the semi-stable degeneration of $S_{\mathrm{GUT}}$ to

$$
\left\{z_{0}=q \cdot\left(u_{0}^{2}-v_{0}^{2}\right)=0\right\} .
$$

$z_{0}$ deposits an $I_{5}$-fiber at the point $\left\{a^{\prime \prime}=0\right\}$ on each fiber of $d P_{a} / B_{2}$ and at the point $\left\{b^{\prime \prime}=0\right\}$ on each fiber of $d P_{b} / B_{2}$. By construction, $\tilde{\zeta}$ and $\tilde{\tau}$ can only meet over $\left\{z_{\delta}=0\right\}$. If

$$
b_{2} \in\left\{u_{1}^{2}-v_{1}^{2}=u_{2}^{2}-v_{2}^{2}=0\right\} \subseteq B_{2}
$$


is any of the 4 fixpoints of the involution $\beta_{2}$, then when $\delta=0$ the fixpoints of the action of $\beta_{3}$ on the fiber $\mathbb{P}_{\left[u_{0}, v_{0}\right]} \times\left\{b_{2}\right\}$ occur at $a=0, \infty$ since $\beta$ takes $a$ to $-a$. We choose the form $q \in H^{0}\left(K_{B_{2}}^{-1}\right)$ in the definition of $z_{0}$ so as not to vanish on any of the fixpoints of the action of $\beta_{2}$ on $B_{2}$.

\section{Geometric model-double cover form}

\subsection{Putting the sections $\zeta$ and $\tau$ of $W_{4} / B_{3}$ on equal footing}

As mentioned earlier, the $\mathbb{Z}_{2}$-action will ultimately incorporate a translation in the fiber direction that will interchange the two distinguished sections $\zeta$ and $\tau$, and so will interchange the two distinguished divisors, $(\zeta)$ and $(\tau)$, to which they correspond. In particular, the Weierstrass forms for the elliptic fibration determined by either of the two sections must be 'on equal footing with the other one' throughout. So we will have to begin from another birational model for $W_{4} / B_{3}$ that achieves the desired 'equal footing'. On each fiber of $W_{4} / B_{3}$, the intersection with the sections $(\zeta)+(\tau)$ distinguishes a $g_{2}^{1}$ (that is, a linear series of projective dimension one and degree two). Taken together the $g_{2}^{1}$ 's yield a $\mathbb{P}^{1}$-bundle

$$
Q:=\mathbb{P}\left(\left(\pi_{B_{3}}\right)_{*} \mathcal{O}_{W_{4}}((\zeta)+(\tau))\right)
$$

over $B_{3}$.

Since we are eventually going to change the elliptic group structure on the torus fibers of $W_{4} / B_{3}$ from the one given by $(\zeta)$ that does not pass through the set of singular points of $W_{4}$ to the one given by $(\tau)$ that actually contains the set of singular points of $W_{4}$, we first want to define a crepant partial resolution of our $F$-theory model $W_{4} / B_{3}$ that becomes a branched double cover of $Q / B_{3}$ and has the property that the proper transform of $(\tau)$ misses the singular set of the resulting fourfold entirely. We achieve this by projecting each torus fiber from the third point $v\left(b_{3}\right)$ of intersection of the line between $\zeta\left(b_{3}\right)$ and $\tau\left(b_{3}\right)$ with the fiber of $W_{4} / B_{3}$ over $b_{3}$.

\subsubsection{Line between the sections}

Over $b_{3} \in\left(B_{3}-S_{\mathrm{GUT}}\right)$, the line through $\zeta\left(b_{3}\right)$ and $\tau\left(b_{3}\right)$ in $\left\{b_{3}\right\} \times_{B_{3}} P$ is given by

$$
x-z^{2} w=0 .
$$

We have denoted the third point of intersection of this line with $\left\{b_{3}\right\} \times_{B_{3}} W_{4}$ as

$$
v\left(b_{3}\right)=\left[w, z^{2} w,\left(-z-a_{420}\right) z^{2} w\right] .
$$

Thus we can modify the defining equation

$$
\left|\begin{array}{cc}
x^{3}+a_{4} z w x^{2}+a_{2} z^{3} w^{2} x+a_{0} z^{5} w^{3} & 1 \\
w y\left(y-\left(a_{5} x+a_{3} z^{2} w\right)\right) & 1
\end{array}\right|=0
$$


of $W_{4}$ by projecting $\left\{b_{3}\right\} \times_{B_{3}} W_{4}$ from the point $v\left(b_{3}\right)$. That is we write

$$
\begin{aligned}
x-z^{2} w & =X \\
y+\left(z+a_{420}\right) z^{2} w & =Y
\end{aligned}
$$

in order that the section $(v)$ correspond to the point $[w, X, Y]=[1,0,0]$. The change of fiber coordinates in $P / B_{3}$ is given by

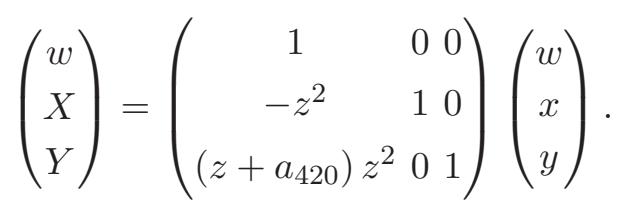

(Notice that this is the identity matrix over a first-order neighborhood of $S_{\mathrm{GUT}}$.) Recalling again that $-a_{53}=a_{420}$, we obtain

$$
\left|\begin{array}{cr}
\left(X^{3}+3 z^{2} w X^{2}+3 z^{4} w^{2} X\right)+a_{4} z w\left(X^{2}+2 z^{2} w X\right)+a_{2} z^{3} w^{2} X & 1 \\
w Y\left(Y-z^{3} w-a_{5} X\right)-\left(z+a_{420}\right) z^{2} w^{2}\left(Y-a_{5} X\right) & 1
\end{array}\right|=0
$$

as the equation for $W_{4}$ in the projective coordinates $[w, X, Y]$.

\subsection{Fundamental projection and modified Weierstrass form}

By considering (6.3) as a quadratic equation in $w$, the Tate form then yields a birationally equivalent double-cover

$$
\bar{W}_{4} / Q
$$

where the branched double cover $\bar{W}_{4}$ of $Q$ is given by the equation

$$
\begin{aligned}
w^{2}= & \left(3 z^{2} X^{2}+a_{4} z X^{2}-Y^{2}+a_{5} X Y\right)^{2} \\
& -4\left(\left(3 z^{4}+\left(2 a_{4}+a_{2}-a_{5}\right) z^{3}-a_{5} a_{420} z^{2}\right) X^{4}+\left(2 z^{3}+a_{420} z^{2}\right) X^{3} Y\right)
\end{aligned}
$$

where the fiber coordinates of $Q / B_{3}$ are given by

$$
\begin{aligned}
& X=x-z^{2} w \\
& Y=y+\left(z+a_{420}\right) z^{2} .
\end{aligned}
$$

(6.4) also allows us to see that $W_{4}$ is birationally a double cover

$$
\pi_{Q}: \bar{W}_{4} \rightarrow Q
$$

with equation

$$
\begin{aligned}
w^{2}= & \left(3 z^{2} X^{2}+a_{4} z X^{2}-Y^{2}+a_{5} X Y\right)^{2} \\
& -4\left(\left(3 z^{4}+\left(2 a_{4}+a_{2}-a_{5}\right) z^{3}-a_{5} a_{420} z^{2}\right) X^{4}+\left(2 z^{3}+a_{420} z^{2}\right) X^{3} Y\right)
\end{aligned}
$$

where

$$
\bar{W}_{4} \subseteq \bar{P}:=\mathbb{P}\left(\mathcal{O}_{B_{3}}(6 N) \oplus \mathcal{O}_{B_{3}}(2 N) \oplus \mathcal{O}_{B_{3}}(3 N)\right)=\mathbb{P}_{[w, X, Y]}
$$


and

$$
Q=\mathbb{P}_{[X, Y]}:=\mathbb{P}\left(\mathcal{O}_{B_{3}}(2 N) \oplus \mathcal{O}_{B_{3}}(3 N)\right)
$$

with branch locus $\Delta$ given by the zeros of the homogeneous polynomial (6.4). Notice that (6.5) is invariant under the transformations

$$
\left[\left(a_{0}, a_{2}, a_{3}, a_{4}, a_{5}\right), z, X, Y, w\right] \mapsto\left[\left(-a_{0},-a_{2},-a_{3},-a_{4},-a_{5}\right),-z, X,-Y, \pm w\right]
$$

so both are possible but only one will leave the holomorphic four-form on $\bar{W}_{4}$ invariant, namely the one that is compatible with the action of $\tilde{\beta}_{4} / \beta_{3}$ that transforms the relative one-form by

$$
\frac{d x}{y} \mapsto-\frac{d x}{y} .
$$

To see which one, we divide (6.5) by $X^{4}$ and define

$$
\vartheta_{0}:=\frac{Y}{X} .
$$

We have the affine equation

$$
\begin{aligned}
w_{0}^{2}= & \left(3 z^{2}+a_{4} z-\vartheta_{0}^{2}+a_{5} \vartheta_{0}\right)^{2} \\
& -4\left(\left(3 z^{4}+\left(2 a_{4}+a_{2}-a_{5}\right) z^{3}-a_{5} a_{420} z^{2}\right)+\left(2 z^{3}+a_{420} z^{2}\right) \vartheta_{0}\right) .
\end{aligned}
$$

Therefore, since $\vartheta_{0} \mapsto-\vartheta_{0}, \tilde{\beta}_{4} / \beta_{3}$ is only compatible with the transformation

$$
\frac{d w_{0}}{\vartheta_{0}} \mapsto-\frac{d w_{0}}{\vartheta_{0}}
$$

on the relative one-form so we conclude that $w_{0}$ must be invariant. Furthermore, since the sections $\zeta$ and $\tau$ are now given by $\{X=0\}$, so, referring to (6.5), their equation becomes

$$
0=w^{2}-Y^{4}=\left(w+Y^{2}\right)\left(w-Y^{2}\right)
$$

and so each of the two sections must be taken to itself under $\tilde{\beta}_{4} / \beta_{3}$.

The canonical bundle of $Q$ is

$$
-2\left(X_{0}\right)-2 N
$$

where $\left(X_{0}\right)$ denotes the divisor $\left\{X_{0}=0\right\}$ whereas the branch locus $\Delta$ has divisor class

$$
4\left(X_{0}\right)+4 N
$$

We therefore replace $W_{4}$ with the birationally equivalent double cover

$$
\pi_{Q}: \bar{W}_{4} \rightarrow Q
$$

branched over $\Delta$ and $\bar{W}_{4}$ is Calabi-Yau. Furthermore

$$
\bar{W}_{4} \cap\{X=0\}=(\bar{\zeta}) \cup(\bar{\tau})
$$

is the union of the proper transforms of the two original sections of $W_{4} / B_{3}$. 


\subsection{The branch locus}

The branch locus $\Delta$ is defined by the equation

$$
\begin{aligned}
0= & \left(\left(3 z^{2}+a_{4} z\right)-\vartheta_{0}\left(\vartheta_{0}-a_{5}\right)\right)^{2} \\
& -4\left(\left(3 z^{4}+\left(2 a_{4}+a_{2}-a_{5}\right) z^{3}-a_{5} a_{420} z^{2}\right)+\left(2 z^{3}+a_{420} z^{2}\right) \vartheta_{0}\right)
\end{aligned}
$$

on the space

$$
Q-\{X=0\}=\left|\mathcal{O}_{B_{3}}(N)\right|,
$$

the total space of the line bundle $\mathcal{O}_{B_{3}}(N)$. Then the equation for

$$
\left(\bar{W}_{4}-\{X=0\}\right) \subseteq(\bar{P}-\{X=0\})
$$

can be rewritten as

$$
\begin{array}{r}
w_{0}^{2}=\left(\left(3 z^{2}+a_{4} z\right)-\vartheta_{0}\left(\vartheta_{0}-a_{5}\right)\right)^{2}-4 a_{420} z^{2}\left(\vartheta_{0}-a_{5}\right) \\
-4 z^{3}\left(3 z+2 a_{4}+a_{2}-\left(\vartheta_{0}+\left(\vartheta_{0}-a_{5}\right)\right)\right) .
\end{array}
$$

Again rescaling the $a_{j}$ and appealing to Bertini's theorem, we will have for general choices of the $a_{j} \in \mathcal{A}$ that singularities of $\Delta$ are supported on the locus

$$
\left\{\vartheta_{0}\left(\vartheta_{0}-a_{5}\right)=z=0\right\} .
$$

Thus $S_{\mathrm{GUT}} \times_{B_{3}} \bar{W}_{4}$ has two components, each isomorphic to $S_{\mathrm{GUT}} \times_{B_{3}} Q$. They are given by

$$
w_{0}=z= \pm \vartheta_{0}\left(\vartheta_{0}-a_{5}\right) .
$$

One of these components, that we will denote as $D_{1}$, intersects $(\zeta)$, while the other, that we will denote as $D_{4}$, intersects $(\tau)$. We write $D_{1}=:\left\{G_{1}=0\right\}$ and $D_{4}=:\left\{G_{4}=0\right\}$ so that

$$
G_{1} G_{4}
$$

on $\bar{W}_{4}$.

Over $\{z=0\}$ the two components coincide as the component $\left\{\vartheta\left(\vartheta_{0}-a_{5}\right)=0\right\}$ locus of the reducible double cover

$$
\left\{w_{0}^{2}=\vartheta_{0}^{2}\left(\vartheta_{0}-a_{5}\right)^{2}\right\}
$$

of

$$
Q \times_{B_{3}}\{z=0\} .
$$

\subsection{The standard $\mathbb{P}^{112}$-formulation}

To relate the presentation (6.4) to the more standard $\mathbb{P}^{112}$-notation used for this type of model, let

$$
\ddot{P}:=\mathbb{P}\left(\mathcal{O}_{B_{3}} \oplus \mathcal{O}_{B_{3}}(2) \oplus \mathcal{O}_{B_{3}}(4)\right)
$$

with coordinates $(w, x, y)$ and write

$$
\begin{aligned}
& X \rightarrow w \\
& Y \rightarrow x \\
& w \rightarrow y+x^{2}
\end{aligned}
$$


so that

$$
\begin{aligned}
w^{2}= & \left(3 z^{2} X^{2}+a_{4} X^{2} z-Y\left(Y-a_{5} X\right)\right)^{2} \\
& -4\left(\left(3 z^{4}+\left(2 a_{4}+a_{2}-a_{5}\right) z^{3}-a_{5} a_{420} z^{2}\right) X^{4}+\left(2 z^{3}+a_{420} z^{2}\right) X^{3} Y\right)
\end{aligned}
$$

becomes

$$
\begin{aligned}
\left(y+x^{2}\right)^{2}= & \left(w^{2}\left(3 z^{2}+a_{4} z\right)-x\left(x-a_{5} w\right)\right)^{2} \\
& -4\left(\left(3 z^{4}+\left(2 a_{4}+a_{2}-a_{5}\right) z^{3}-a_{5} a_{420} z^{2}\right) w^{4}+\left(2 z^{3}+a_{420} z^{2}\right) x w^{3}\right)
\end{aligned}
$$

that can be rewritten as

$$
\begin{aligned}
y^{2}+2 x^{2} y= & -2 a_{5} x^{3} w \\
& +\left(a_{5}^{2}-2 a_{4} z-6 z^{2}\right) x^{2} w^{2} \\
& +\left(2 a_{5} a_{4} z+\left(6 a_{5}-4 a_{420}\right) z^{2}-8 z^{3}\right) x w^{3} \\
& -\left(\left(-a_{4}^{2}-4 a_{5} a_{420}\right) z^{2}+\left(2 a_{4}+4 a_{2}-4 a_{5}\right) z^{3}+3 z^{4}\right) w^{4} .
\end{aligned}
$$

We include appendix A.1 by Sakura Schäfer-Nameki containing a brief overview of elliptically-fibered Calabi-Yau manifolds symmetric with respect to two sections.

\section{Desingularization of $\bar{W}_{4}$}

\subsection{Localizing at the singularities of $\bar{W}_{4}$}

We first rearrange terms of (6.7) in increasing order of total degree in the variables $\left(z, \vartheta_{0}\right)$ so that the branch locus $\Delta$ is given by

$$
\begin{aligned}
& \left(a_{5} \vartheta_{0}+a_{4} z\right)^{2}+4 a_{5} a_{420} z^{2} \\
& -2\left(a_{5} \vartheta_{0}^{3}+a_{4} \vartheta_{0}^{2} z\right)+\left(6 a_{5}-4 a_{420}\right) z^{2} \vartheta_{0}+\left(-2 a_{4}-4 a_{2}+4 a_{5}\right) z^{3} \\
& +\vartheta_{0}^{4}-6 \vartheta_{0}^{2} z^{2}-8 z^{3} \vartheta_{0}-3 z^{4} .
\end{aligned}
$$

$\Delta$ will have ordinary nodal singularities along $\left\{z=\vartheta_{0}=0\right\}$ except where the quadratic normal cone

$$
\left(a_{5} \vartheta_{0}+a_{4} z\right)^{2}+4 a_{5} a_{420} z^{2}=0
$$

is not of maximal rank, namely where

$$
a_{5} a_{420}=0 .
$$

If $a_{5} a_{420}=0$, the reduced quadratic cone is given by

$$
\left[\vartheta_{0}, z\right]=\left[a_{4},-a_{5}\right] .
$$

on which the cubic cone evaluates as

$$
a_{5}^{2}\left(a_{420} a_{4}+\left(2 a_{4}+a_{2}+a_{5}\right) a_{5}\right)=0 .
$$


So the quadratic and cubic cone both vanish identically over $\left\{a_{5}=0\right\}$ but both only vanish over $\left\{a_{420}=a_{53}=0\right\}$ when additionally

$$
a_{5}^{3}\left(a_{4}-a_{0}+a_{5}\right)=0
$$

a locus that has only finite intersection with $S_{\mathrm{GUT}}$.

We next rearrange terms of (6.7) in increasing order of total degree in the variables $\left(z, a_{5}-\vartheta_{0}\right)$. The branch locus $\Delta$ is then given by

$$
\begin{aligned}
0= & \left(a_{5}\left(a_{5}-\vartheta_{0}\right)+a_{4} z\right)^{2} \\
& -2 a_{5}\left(a_{5}-\vartheta_{0}\right)^{3}-2 a_{4} z\left(a_{5}-\vartheta_{0}\right)^{2}+\left(4 a_{420}+6 a_{5}\right)\left(a_{5}-\vartheta_{0}\right) z^{2}-\left(2 a_{4}-4 a_{2}-4 a_{5}\right) z^{3} \\
& +\left(a_{5}-\vartheta_{0}\right)^{4}-6\left(a_{5}-\vartheta_{0}\right)^{2} z^{2}+8 z^{3}\left(a_{5}-\vartheta_{0}\right)-3 z^{4} .
\end{aligned}
$$

The quadratic normal cone along $\left\{z=a_{5}-\vartheta_{0}=0\right\}$ is of rank one except where $a_{5}=a_{4}=$ 0 . It is spanned by the vector $\left(a_{5}-\vartheta_{0}, z\right)=\left(a_{4},-a_{5}\right)$. Evaluating the cubic normal cone along this vector and recalling that $a_{420}=-a_{53}$ gives

$$
\left(-a_{4} a_{3}+a_{5}\left(a_{0}+a_{3}\right)\right) a_{5}^{2}=0 .
$$

Thus a simple modification will resolve the singularity of $\bar{W}_{4}$ over $\left\{a_{5}-\vartheta_{0}=z=0\right\}$ unless $a_{5}=0$ or

$$
\left|\begin{array}{cc}
a_{4} & -a_{5} \\
a_{0}+a_{3} & a_{3}
\end{array}\right|=0
$$

\subsection{Resolving the singularities of $\bar{W}_{4}$}

\subsubsection{First modification of $\Delta$}

We first make the modification of $\Delta$ and $\bar{W}_{4}$ at $\{\vartheta=z=0\}$. Namely, inside

$$
Q^{(1)}:=\left\{\left|\begin{array}{cc}
\vartheta_{0} & z \\
\vartheta_{1} & Z_{14}
\end{array}\right|\right\} \subseteq Q \times \mathbb{P}_{\left[\vartheta_{1}, Z_{14}\right]}
$$

we write

$$
Z_{0}:=\frac{\vartheta_{0}}{\vartheta_{1}}=\frac{z}{Z_{14}}
$$

The proper transform $\Delta^{(1)}$ of $\Delta$ in $Q^{(1)}$ is given by the equation

$$
\begin{aligned}
0= & \left(a_{5} \vartheta_{1}+a_{4} Z_{14}\right)^{2}+4 a_{5} a_{420} Z_{14}^{2} \\
& +\left(-2 a_{5} \vartheta_{1}^{3}-2 a_{4} \vartheta_{1}^{2} Z_{14}+\left(6 a_{5}-4 a_{420}\right) \vartheta_{1} Z_{14}^{2}-\left(2 a_{4}+4 a_{2}-4 a_{5}\right) Z_{14}^{3}\right) Z_{0} \\
& +\left(\vartheta_{1}^{4}-6 \vartheta_{1}^{2} Z_{14}^{2}-8 Z_{14}^{3} \vartheta_{1}-3 Z_{14}^{4}\right) Z_{0}^{2}
\end{aligned}
$$

Over $z=Z_{14} Z_{0}=0$ we have two possible singular loci in $\Delta^{(1)}$. These loci are given respectively by

$$
\left\{Z_{14}=\left(\vartheta_{1} Z_{0}-a_{5}\right)^{2}=0\right\}
$$


where $Z_{14}=0$ and singular points of

$$
\left\{Z_{0}=\left(a_{5} \vartheta_{1}+a_{4} Z_{14}\right)^{2}+4 a_{5} a_{420} Z_{14}^{2}=0\right\}
$$

at which $Z_{0}=0$ and $Z_{14} \neq 0$. In this last case we write

$$
Z_{0}=\left(a_{5} \frac{\vartheta_{1}}{Z_{14}}+a_{4}\right)^{2}+4 a_{5} a_{420}=0
$$

to conclude that singular points can only occur where the discriminant

$$
\left\{4 a_{5} a_{420}=0\right\}
$$

of the quadratic equation in $\frac{\vartheta_{1}}{Z_{14}}$ is singular, that is, where $Z_{0}=a_{5}=a_{420}=0$. Substituting in (7.5) we conclude that, in addition, $a_{4}=0$. Since $\frac{\partial}{\partial Z_{0}}$ applied to (7.5) and evaluated at such singular points would also have to vanish, we would also have $a_{2}=0$ and so $a_{0}=0$ contradicting the assumption of a generic allowable selection of the $a_{j}$ and $z$ in the linear system $|\mathcal{A}|$.

Lemma 4. Singularities of the branch locus $\Delta^{(1)}$ lie on locus

$$
\left\{Z_{14}=\left(\vartheta_{1} Z_{0}-a_{5}\right)^{2}=0\right\} .
$$

The assumption that the curve $\left\{a_{5}=z=0\right\} \subseteq B_{3}$ is smooth can be weakened to allow nodal singularities. Since $a_{4}=0$ there, the potential nodal singularities in $\Delta^{(1)}$ thereby created are in fact already resolved by the fact that the entire fiber of $Z_{0}$ already lies in $\Delta^{(1)}$ over any point where $a_{5}=a_{4}=0$. In fact, in section 6.3 of [15] we impose a normal-crossing assumption on $\left\{a_{5}=z=0\right\} \subseteq B_{3}$.

The proper transform $\Delta^{(1)}$ of $\Delta$ in $Q^{(1)}$ is the total transform minus twice the exceptional divisor, so that the proper transform is again twice the anticanonical divisor of $Q^{(1)}$. So the branched double cover $W_{4}^{(1)}$ given by the equation

$$
\begin{aligned}
w_{1}^{2}= & \left(a_{5} \vartheta_{1}+a_{4} Z_{14}\right)^{2}+4 a_{5} a_{420} Z_{14}^{2} \\
& +\left(-2 a_{5} \vartheta_{1}^{3}-2 a_{4} \vartheta_{1}^{2} Z_{14}+\left(6 a_{5}+4 a_{420}\right) \vartheta_{1} Z_{14}^{2}-\left(2 a_{4}+4 a_{2}-4 a_{5}\right) Z_{14}^{3}\right) Z_{0} \\
& +\left(\vartheta_{1}^{4}-6 \vartheta_{1}^{2} Z_{14}^{2}-8 Z_{14}^{3} \vartheta_{1}-3 Z_{14}^{4}\right) Z_{0}^{2} .
\end{aligned}
$$

is again Calabi-Yau. We retain the notation $D_{1}$ and $D_{4}$ for the proper transforms in $W_{4}^{(1)}$ of those respective divisors in $\bar{W}_{4}$, namely the two components of $W_{4}^{(1)} \times_{\Delta^{(1)}}\left\{Z_{14}=0\right\}$. We denote the irreducible branched double cover $W_{4}^{(1)} \times_{Q^{(1)}}\left\{Z_{0}=0\right\}$ as $D_{0}$. Over $\left\{z=a_{5} a_{420}=0\right\}, D_{0}$ splits into two components, $\left\{w_{1}= \pm\left(a_{5} \vartheta_{1}+a_{4} Z_{14}\right)\right\}$, and each component projects isomorphically to $\left\{Z_{0}=0\right\} \subseteq Q^{(1)}$.

\subsubsection{Second modification of $\Delta$}

Recalling that $z=Z_{0} Z_{14}$ we must next attend to the singularities of $\Delta^{(1)}$ lying in $\left\{Z_{14}=0\right\}$. As we have seen in the last subsection, these lie on the locus $\left\{a_{5}-\vartheta_{1} Z_{0}=Z_{14}=0\right\}$ that only intersects $D_{0}$ over $\left\{a_{5}=Z_{0}=0\right\}$. 
First, referring to (7.5), we rewrite the equation for $\Delta^{(1)}$ in terms of the variables $\left(\left(a_{5}-\vartheta_{1} Z_{0}\right), Z_{14}\right)$ as

$$
\begin{aligned}
0= & \left(\vartheta_{1}\left(a_{5}-\vartheta_{1} Z_{0}\right)+a_{4} Z_{14}\right)^{2} \\
& \left(6 a_{5}+4 a_{420}\right)\left(a_{5}-\vartheta_{1} Z_{0}\right) Z_{14}^{2}-\left(4 a_{5}+2 a_{4}+4 a_{2}\right) Z_{14}^{3} Z_{0} \\
& -6\left(a_{5}-\vartheta_{1} Z_{0}\right)^{2} Z_{14}^{2}+8\left(\vartheta_{1} Z_{0}-a_{5}\right) Z_{14}^{3} Z_{0}-3 Z_{14}^{4} Z_{0}^{2}
\end{aligned}
$$

Next define

$$
Q^{(2)}:=\left\{\left|\begin{array}{cc}
a_{5}-\vartheta_{1} Z_{0} & Z_{14} \\
\vartheta_{2} & \tilde{Z}_{14}
\end{array}\right|=0\right\} \subseteq Q^{(1)} \times \mathbb{P}_{\left[\vartheta_{2}, \tilde{Z}_{14}\right]}
$$

and

for which we have

$$
Z_{23}:=\frac{a_{5}-\vartheta_{1} Z_{0}}{\vartheta_{2}}=\frac{Z_{14}}{\tilde{Z}_{14}}
$$

$$
\pi^{(2)}: Q^{(2)} \rightarrow B_{3} .
$$

The equation for the proper transform $\Delta^{(2)}$ of $\Delta^{(1)}$ becomes

$$
\begin{aligned}
0= & \left(\vartheta_{1} \vartheta_{2}+a_{4} \tilde{Z}_{14}\right)^{2} \\
& +\left(\left(6 a_{5}+4 a_{420}\right) \vartheta_{2}-\left(4 a_{5}+2 a_{4}+4 a_{2}\right) Z_{0} \tilde{Z}_{14}\right) \tilde{Z}_{14}^{2} Z_{23} \\
& -\left(6 \vartheta_{2}^{2}-8 \vartheta_{2} Z_{0} \tilde{Z}_{14}+3 \tilde{Z}_{14}^{2} Z_{0}^{2}\right) \tilde{Z}_{14}^{2} Z_{23}^{2} .
\end{aligned}
$$

The proper transform $\Delta^{(2)}$ in $Q^{(2)}$ is the total transform minus twice the exceptional divisor, so that the proper transform is again twice the anticanonical divisor of $Q^{(2)}$. Therefore we have a Calabi-Yau fourfold $W_{4}^{(2)}$ given by the completion over $\{X=0\}$ of the solution set of

$$
\begin{aligned}
w_{2}^{2}= & \left(\vartheta_{1} \vartheta_{2}+a_{4} \tilde{Z}_{14}\right)^{2} \\
& +\left(\left(6 a_{5}+4 a_{420}\right) \vartheta_{2}-\left(4 a_{5}+2 a_{4}+4 a_{2}\right) Z_{0} \tilde{Z}_{14}\right) \tilde{Z}_{14}^{2} Z_{23} \\
& -\left(6 \vartheta_{2}^{2}-8 \vartheta_{2} Z_{0} \tilde{Z}_{14}+3 \tilde{Z}_{14}^{2} Z_{0}^{2}\right) \tilde{Z}_{14}^{2} Z_{23}^{2} .
\end{aligned}
$$

Over the exceptional locus, given by $\left\{Z_{23}=0\right\} \subseteq Q^{(2)}$, the equation for $\Delta^{(2)}$ is a perfect square so that $W_{4}^{(2)} \times_{B_{3}} S_{\mathrm{GUT}}$ splits into five components. Two of these are new components lying over $\left\{Z_{23}=0\right\} \subseteq Q^{(2)}$ that we call $D_{2}$ and $D_{3}$. In addition we have lifted components, the two components that we continue to call $D_{1}$ and $D_{4}$ lying over $\left\{\tilde{Z}_{14}=0\right\} \subseteq Q^{(2)}$, and finally the lifted component over $\left\{Z_{0}=0\right\}$ that we continue to call $D_{0}$. We number things so that, over a general point of $S_{\mathrm{GUT}}, D_{2}$ intersects $D_{1}$ and $D_{3}$ intersects $D_{4}$. The incidence of the five components over a general point of $S_{\mathrm{GUT}}$ is that of the extended Dynkin diagram $\tilde{A}_{4}$.

By lemma 4 singular points of $\Delta^{(2)}$ can only occur where $Z_{23}=0$, so $\tilde{Z}_{14} \neq 0$ there and these points can be singular points of $\Delta^{(2)}$ only if $\frac{\vartheta_{1} \vartheta_{2}}{\tilde{Z}_{14}}=-a_{4}$ and

$$
\frac{\vartheta_{2}}{\tilde{Z}_{14}}=\frac{\left(2 a_{4}+4 a_{2}+4 a_{5}\right) Z_{0}}{6 a_{5}+4 a_{420}} .
$$


Multiplying both sides of this last equation by $\vartheta_{1}$ and substituting, then recalling that at these points $a_{5}-\vartheta_{1} Z_{0}=0$ and that $a_{54320}=0$, we obtain

$$
a_{5} a_{0}+a_{3}\left(a_{5}+a_{4}\right)=0
$$

that can be rewritten as the relation

$$
Z_{0} \cdot\left|\begin{array}{cc}
a_{4} & -a_{5} \\
a_{0}+a_{3} & a_{3}
\end{array}\right|=0
$$

given in (7.4).

Lemma 5. Singularities of the branch locus $\Delta^{(2)}$ only occur over

$$
z=\left|\begin{array}{cc}
a_{4} & -a_{5} \\
a_{0}+a_{3} & a_{3}
\end{array}\right|=0
$$

and then only where

$$
\frac{\vartheta_{2}}{\tilde{Z}_{14}}=\frac{\left(a_{4}+2 a_{2}+2 a_{5}\right) Z_{0}}{a_{5}-2 a_{3}} .
$$

Over a general point of $\left\{z=a_{5}=0\right\}, D_{0}$ splits into the two components $D_{01}+D_{04}$ that reconfigure with the specialization of $D_{2}+D_{3}$ over $\left\{z=a_{5}=0\right\}$ to form the extended Dynkin diagram for $D_{5}$ as is shown by the relation

$$
\vartheta_{1} Z_{0}+\vartheta_{2} Z_{23}=a_{5}
$$

Over a general point of $\left\{z=a_{420}=a_{53}=0\right\}$ again $D_{0}$ splits into two components, augmenting the extended Dynkin diagram $\tilde{A}_{4}$ to the extended Dynkin diagram $\tilde{A}_{5}$.

\subsubsection{Singularities of higher codimension}

We have seen in lemma 5 that, at singular points of $\Delta^{(2)}, Z_{23}=0$ and $\tilde{Z}_{14} \neq 0$ and they lie over

$$
\left\{z=\left|\begin{array}{cc}
a_{4} & -a_{5} \\
a_{0}+a_{3} & a_{3}
\end{array}\right|=0\right\} \subseteq B_{3} .
$$

In fact, if one rewrites (7.9) in the form

$$
\left|\begin{array}{cc}
w_{2}-\left(\vartheta_{1} \vartheta_{2}+a_{4} \tilde{Z}_{14}\right) & \tilde{Z}_{14}^{2} Z_{23} \\
-A & w_{2}+\left(\vartheta_{1} \vartheta_{2}+a_{4} \tilde{Z}_{14}\right)
\end{array}\right|=0
$$

where

$$
\begin{array}{r}
-A=\left(\left(6 a_{5}+4 a_{420}\right) \vartheta_{2}-\left(4 a_{5}+2 a_{4}+4 a_{2}\right) Z_{0} \tilde{Z}_{14}\right) \\
-\left(6 \vartheta_{2}^{2}-8 \vartheta_{2} Z_{0} \tilde{Z}_{14}+3 \tilde{Z}_{14}^{2} Z_{0}^{2}\right) Z_{23}
\end{array}
$$


over $\left\{\tilde{Z}_{14}=0\right\}$ the equation of $W_{4}^{(2)}$ becomes

$$
\left(w_{2}-\vartheta_{1} \vartheta_{2}\right)\left(w_{2}+\vartheta_{1} \vartheta_{2}\right)=0 \text {. }
$$

Recalling that $\tilde{Z}_{14}$ and $\vartheta_{1} \vartheta_{2}$ cannot vanish simultaneously, one sees that

$$
C_{\overline{\mathbf{5}}}^{(44)} \cap\left\{\tilde{Z}_{14}=0\right\}=\emptyset
$$

where $C_{\overline{5}}^{(44)}$ is given by the vanishing

$$
\begin{aligned}
w_{2}= & \vartheta_{1} \vartheta_{2}+a_{4} \tilde{Z}_{14}=Z_{23}= \\
& \left(a_{5}-2 a_{3}\right) \vartheta_{2}-\left(a_{4}+2 a_{2}+2 a_{5}\right) Z_{0} \tilde{Z}_{14}=0
\end{aligned}
$$

of all four entries in the $2 \times 2$ matrix (7.11) is a simple nodal locus of $W_{4}^{(2)}$. Since $\tilde{Z}_{14}$ is never zero along $C_{\overline{5}}^{(44)}$ we conclude that

$$
\left(D_{1} \cup D_{4}\right) \cap C_{\overline{5}}^{(44)}=\emptyset .
$$

The entries in (7.11) are all invariant under the action of $\tilde{\beta}_{4} / \beta_{3}$. Therefore we can take either small blow-up to complete the crepant resolution.

\subsection{The smooth model $\tilde{W}_{4}$}

After taking the small resolution of the nodal curve $C_{\overline{\mathbf{5}}}^{(44)}$ in $W_{4}^{(2)}$ as described just above, we obtain the inclusion of proper transform

$$
\tilde{\Delta} \subseteq \tilde{W}_{4}
$$

where $\tilde{\Delta}$ is non-singular.

We retain the notations $D_{j}, j=0, \ldots, 4$, for the proper transforms in $\tilde{W}_{4}$ of the corresponding divisors in $W_{4}^{(2)}$. We will let $G_{j}$ denote the canonical section of the line bundle determined by the divisor $D_{j}$, that is

$$
D_{j}=\left\{G_{j}=0\right\} .
$$

Remark 4. The exceptional divisors $D_{j}$ are identified with the positive simple roots of $\mathrm{SU}(5)_{\text {gauge }}$ in such a way that the involution $\beta_{3}$ on $B_{3}$ and the action

$$
\frac{w_{0}}{\vartheta_{0}} \mapsto-\frac{w_{0}}{\vartheta_{0}}
$$

on the fibers of $\tilde{W}_{4} / B_{3}$ induces the non-trivial geometric action

$$
\begin{aligned}
D_{0}=-\left(D_{1}+D_{2}+D_{3}+D_{4}\right) & \mapsto-D_{0} \\
D_{1} & \mapsto-D_{4} \\
D_{2} & \mapsto-D_{3} \\
D_{3} & \mapsto-D_{2} \\
D_{4} & \mapsto-D_{1}
\end{aligned}
$$


on the exceptional fibers of $\tilde{W}_{4}$. As explained in [14], the action of $\tilde{\beta}_{4}$ on the roots $D_{1}, \ldots, D_{4}$ reverses the choice of positive Weyl chamber used in making the dictionary between exceptional divisors of $\tilde{W}_{4}$ and the SU(5) gauge-roots. This reversal of positive Weyl chamber exactly reverses the non-trivial involution (7.14) thereby preserving the

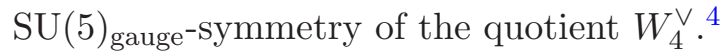

The identity

$$
a_{5}=Z_{0} \vartheta_{1}+Z_{23} \vartheta_{2}
$$

intertwines $D_{0}$ and $D_{2}+D_{3}$ above $\left\{z=a_{5}=0\right\}$. After the first modification, the equation of the spectral variety on $\left\{Z_{0}=0\right\}$ is

$$
\left(a_{5} \vartheta_{1}+a_{4} Z_{14}\right)^{2}-4 a_{5} a_{420} Z_{14}^{2}=0
$$

whereas the equations of the center of the second modification are

$$
a_{5}-\vartheta_{1}=Z_{14}=0
$$

Since $\vartheta_{1}$ and $Z_{14}$ cannot vanish simultaneously, therefore after the second modification, the proper transform of $\left\{Z_{0}=0\right\}$ will only intersect $\left\{Z_{23}=0\right\}$ along the fiber of $\left\{Z_{23}=0\right\}$ over $\left\{z=a_{5}=0\right\}$. If the modifications were done in the opposite order, the divisors $\left\{Z_{0}=0\right\}$ and $\left\{Z_{23}=0\right\}$ in $\tilde{\Delta}$ would have been flopped.

\subsection{The spectral divisor in $\tilde{W}_{4}$}

Referring to (3.1), the spectral divisor is obviously invariant under the involution $(x, y) \mapsto$ $(x,-y)$. To compute the image

$$
\mathcal{C}_{0}^{(4)}+\mathcal{C}_{0}^{(1)} \subseteq Q
$$

of $\mathcal{D}^{(4)}+\mathcal{D}^{(1)}$ we write

$$
t=\frac{y}{x}=\frac{Y-\left(z+a_{420}\right) z^{2} w}{X+z^{2} w}
$$

so that

$$
t-z=\frac{Y-z X-\left(2 z+a_{420}\right) z^{2} w}{X+z^{2} w}
$$

and

$$
t+z=\frac{Y+z X-a_{420} z^{2} w}{X+z^{2} w} .
$$

So, setting $\frac{X}{z^{2} w}=1$ and $\frac{Y}{z^{2} w}=\vartheta$, the affine equations

$$
\begin{aligned}
\left(\vartheta-\left(z+a_{420}\right)\right)^{2}\left(a_{5}\left(\vartheta-\left(z+a_{420}\right)\right)^{2}+a_{54}\left(z\left(\vartheta-2\left(z+a_{420}\right)\right)\right)-4 a_{20} z^{2}\right) & \\
-8 a_{0} z^{3}\left(z\left(\vartheta+z-a_{420}\right)\right) & =0
\end{aligned}
$$

and

$$
\vartheta-\left(3 z+a_{420}\right)=0
$$

\footnotetext{
${ }^{4}$ This assertion is reflected in the fact that the action of $\tilde{\beta}_{4}$ leaves all entries of equation (4.10) of [14] invariant.
} 
are the respective equations for the image $\mathcal{C}_{0}^{(4)}+\mathcal{C}_{0}^{(1)} \subseteq Q$ of the components of the spectral divisor. Setting $z=0$ we obtain

$$
\begin{aligned}
a_{5}\left(\vartheta_{0}-a_{420}\right)^{4} & =0 \\
\vartheta_{0}-a_{420} & =0
\end{aligned}
$$

consistent with the fact that the inverse image of both $\mathcal{C}_{0}^{(4)}$ and $\mathcal{C}_{0}^{(1)}$ in $\bar{W}_{4}$ are reducible, only one of its two components correspond to the image

$$
\overline{\mathcal{C}}_{\text {Higgs }}=\overline{\mathcal{C}}_{\text {Higgs }}^{(4)}+\overline{\mathcal{C}}_{\text {Higgs }}^{(1)} \subseteq \bar{W}_{4}
$$

of $\mathcal{D}^{(4)}+\mathcal{D}^{(1)}$ and only $\mathcal{C}_{0}^{(4)}$ intersects the proper transform $\{X=0\}$ of $(\zeta)+(\tau)$ and it only intersects simply along one of the two sections. We let

$$
\mathcal{C}_{\text {Higgs }}=\mathcal{C}_{\text {Higgs }}^{(4)}+\mathcal{C}_{\text {Higgs }}^{(1)} \subseteq \tilde{Q}=Q^{(2)}
$$

denote the proper (also the total) transform of (7.17) and

$$
\tilde{\mathcal{C}}_{\text {Higgs }} \subseteq \tilde{W}_{4}
$$

denote the proper (also the total) transform of $\overline{\mathcal{C}}_{\text {Higgs }}$. Then

$$
\mathcal{C}_{\text {Higgs }}:=\left(\pi_{\tilde{Q}}\right)_{*}\left(\tilde{\mathcal{C}}_{\text {Higgs }}\right) .
$$

The equations

$$
\begin{aligned}
z & =Z_{0} \tilde{Z}_{14} Z_{23} \\
\vartheta-a_{420} & =\vartheta_{1} Z_{0}-a_{420}
\end{aligned}
$$

imply that $\mathcal{C}_{\text {Higgs }}$ is the total transform of $\mathcal{C}_{0}$ so that

$$
\mathcal{C}_{\text {Higgs }} \times_{B_{3}} S_{\mathrm{GUT}} \subseteq\left\{\tilde{Z}_{14}=0\right\} .
$$

Therefore $\vartheta_{1} \vartheta_{2}$ never vanishes on $\mathcal{C}_{\mathrm{Higgs}} \times{ }_{B_{3}} S_{\mathrm{GUT}}$, and this in turn implies that neither component of $\tilde{\mathcal{C}}_{\text {Higgs }} \times_{B_{3}} S_{\mathrm{GUT}}$ can lie in the inherited component $D_{1} \subseteq \tilde{W}_{4}$ containing the proper transform of $(\zeta)$. Thus

$$
\tilde{\mathcal{C}}_{\text {Higgs }} \times_{B_{3}} S_{\text {GUT }} \subseteq\left\{D_{4}=0\right\} .
$$

Finally, by $(7.15), \mathcal{C}_{\text {Higgs }}^{(4)}+\mathcal{C}_{\text {Higgs }}^{(1)}$ has homology class is

$$
\left(4\left(X_{2}\right)+5 N\right)+\left(\left(X_{2}\right)+N\right) .
$$

\subsubsection{Locating matter curves and the Higgs curve}

Referring to (7.16) $S_{\mathrm{GUT}} \subseteq B_{3}$ is lifted into $\tilde{W}_{4}$ via

$$
\left\{\vartheta_{1} Z_{0}-a_{420}=\tilde{Z}_{14}=0\right\} \subseteq \tilde{Q}
$$


with the property that $\vartheta_{1}$ is never zero on the lifting. The matter and Higgs curves will all lie in this lifting.

Therefore, assuming that no component lies in $\left\{a_{420}=z=0\right\}$,

$$
\Sigma_{\mathbf{1 0}}^{(4)}=\left\{a_{5}=\tilde{Z}_{14}=\vartheta_{1} Z_{0}-a_{420}=0\right\} \subseteq \mathcal{C}_{\text {Higgs }} .
$$

Since $\vartheta_{1}$ cannot vanish when $\tilde{Z}_{14}=0$,

$$
\Sigma_{\overline{\mathbf{5}}}^{(41)}:=\left\{Z_{0}=a_{420}=\tilde{Z}_{14}=0\right\} \subseteq \mathcal{C}_{\text {Higgs }}^{(4)} .
$$

By (7.12) and (7.13) the singular curve $C_{\overline{\mathbf{5}}}^{(44)}$ determining the Higgs curve must also lie on

$$
\begin{aligned}
& \vartheta_{1} \vartheta_{2}+a_{4} \tilde{Z}_{14}=Z_{23}= \\
& \quad\left(a_{5}-2 a_{3}\right) \vartheta_{2}-\left(a_{4}+2 a_{2}-2 a_{5}\right) Z_{0} \tilde{Z}_{14}=0
\end{aligned}
$$

as well as

$$
\left\{\left|\begin{array}{cc}
a_{4} & -a_{5} \\
a_{0}+a_{3} & a_{3}
\end{array}\right|=0\right\} \cap\left\{Z_{23}=0\right\}
$$

so that $\vartheta_{1} \vartheta_{2} \neq 0$ and, so by lemma 5

$$
\frac{\vartheta_{2}}{\tilde{Z}_{14}}=\frac{\left(a_{4}+2 a_{2}+2 a_{5}\right) Z_{0}}{a_{5}-2 a_{3}}
$$

and therefore

$$
\Sigma_{\overline{\mathbf{5}}}^{(44)}:=\left\{\left|\begin{array}{cc}
a_{4} & a_{5} \\
a_{0}+a_{3} & -a_{3}
\end{array}\right|=\tilde{Z}_{14}=\vartheta_{1} Z_{0}-a_{420}=0\right\} \subseteq \mathcal{C}_{\text {Higgs }}^{(4)} .
$$

Furthermore the two values

$$
\frac{\tilde{t}}{\tilde{z}}= \pm \sqrt{\frac{a_{0}}{a_{54}}}
$$

in (3.10) lie in $\mathcal{C}_{\text {Higgs }}^{(4)}$ does not meet the singular curve $C_{\mathbf{4 4}} \subseteq \tilde{\Delta}$. The lifted spectral curves $\tilde{\Sigma}_{\mathbf{1 0}}^{(4)}, \tilde{\Sigma}_{\overline{\mathbf{5}}}^{(41)}, \tilde{\Sigma}_{\overline{\mathbf{5}}}^{(44)} \subseteq \tilde{\mathcal{C}}_{\text {Higgs }}$ and by (7.19) have associated spectral surfaces given by

$$
\begin{aligned}
& \tilde{E}_{\mathbf{1 0}}^{(4)}:=\tilde{\Sigma}_{\mathbf{1 0}}^{(4)} \times_{S_{\mathrm{GUT}}} D_{4} \cong E_{\mathbf{1 0}}^{(4)}:=\Sigma_{\mathbf{1 0}}^{(4)} \times_{S_{\mathrm{GUT}}}\left\{\tilde{Z}_{14}=0\right\} . \\
& \tilde{E}_{\overline{\mathbf{5}}}^{(41)}:=\tilde{\Sigma}_{\overline{\mathbf{5}}}^{(41)} \times_{S_{\mathrm{GUT}}} D_{4} \cong \Sigma_{\overline{\mathbf{5}}}^{(41)}:=\Sigma_{\overline{\mathbf{5}}}^{(41)} \times_{S_{\mathrm{GUT}}}\left\{\tilde{Z}_{14}=0\right\} \\
& \tilde{E}_{\overline{\mathbf{5}}}^{(44)}:=\tilde{\Sigma}_{\overline{\overline{5}}}^{(44)} \times_{S_{\mathrm{GUT}}} D_{4} \cong \Sigma_{\overline{\mathbf{5}}}^{(44)}:=\Sigma_{\overline{\overline{5}}}^{(44)} \times_{S_{\mathrm{GUT}}}\left\{\tilde{Z}_{14}=0\right\} .
\end{aligned}
$$

\subsection{Topology of $\tilde{W}_{4}$}

\subsubsection{Picard groups}

The Picard group of $\tilde{Q}$ is freely generated by generators

$$
\begin{aligned}
& \pi_{B_{3}}^{*}\left(\operatorname{Pic}\left(B_{3}\right)\right) \\
& (X),\left(Z_{0}\right),\left(Z_{23}\right)
\end{aligned}
$$


where $(X)$ denotes the divisor given by $X_{0}=0$, etc. The Picard group of $\tilde{W}_{4}$ is generated by

$$
\pi_{\tilde{Q}}^{*} \operatorname{Pic}(\tilde{Q}),(\tilde{\zeta}),(\tilde{\tau}), D_{0}, \ldots, D_{4}
$$

with relations

$$
\begin{aligned}
\pi_{\tilde{Q}}^{*}\left(\left(X_{0}\right)\right) & =(\tilde{\zeta})+(\tilde{\tau}) \\
\pi_{\tilde{Q}}^{*}\left(\left(Z_{34}\right)\right) & =D_{2}+D_{3} \\
\pi_{\tilde{Q}}^{*}\left(\left(Z_{1}\right)\right) & =D_{0} \\
\tilde{\pi}_{B_{3}}^{*}\left(S_{\mathrm{GUT}}\right) & =\sum_{j=0}^{4} D_{j} .
\end{aligned}
$$

The cohomology class of $\tilde{\Delta} \subseteq \tilde{Q}$ is given by

$$
4\left(\left(X_{2}\right)+N\right)-2\left(\left(Z_{0}\right)+\left(Z_{23}\right)\right)
$$

and

$$
K_{\tilde{Q}}=-\left(2\left(X_{2}\right)+2 N\right)+\left(\left(Z_{0}\right)+\left(Z_{23}\right)\right)
$$

where, as before, $N=\tilde{\pi}^{*}\left(c_{1}(\mathcal{N})\right)$ and $\mathcal{N}$ is the line bundle on $B_{3}$ whose sections include $z$ and the $a_{j}$. Thus the Picard group of $\tilde{\Delta}$ admits the effective square root

$$
2\left(\left(X_{2}\right)+N\right)-\left(\left(Z_{0}\right)+\left(Z_{23}\right)\right)
$$

of the branch locus of $\pi_{\tilde{Q}}$. Also

$$
\begin{aligned}
\mathcal{C}_{\text {Higgs }} & =\mathcal{C}_{\text {Higgs }}^{(4)}+\mathcal{C}_{\text {Higgs }}^{(1)}=\mathcal{C}_{\text {Higgs }}^{(4)}+\operatorname{image}(\tilde{\tau}) \\
& \equiv 5\left(X_{2}\right)+5 N \in \operatorname{Pic}(\tilde{Q})
\end{aligned}
$$

We have the following linear equivalences on $\tilde{Q}$ :

$$
\begin{aligned}
\left(\vartheta_{0}\right) & =\left(Y_{0}\right)-\left(X_{0}\right) \equiv N \\
\left(\vartheta_{1}\right) & =\left(Y_{1}\right)-\left(X_{1}\right)=\left(\left(Y_{0}\right)-\left(Z_{0}\right)\right)-\left(X_{0}\right) \\
\left(\vartheta_{2}\right) & =\left(Y_{2}\right)-\left(X_{2}\right)=\left(\left(Y_{0}\right)-\left(Z_{23}\right)\right)-\left(X_{0}\right) \\
\left(\vartheta_{1}\right)+\left(Z_{0}\right) & =\left(\vartheta_{2}\right)+\left(Z_{23}\right) \equiv N .
\end{aligned}
$$

Finally, since none of the blow-ups in the resolution over $B_{3}$ touch $\left(X_{0}\right)$, from now on we will simply identify

$$
(X):=\left(X_{0}\right)=\left(X_{1}\right)=\left(X_{2}\right) .
$$

\subsubsection{Intersections in $Q^{(2)}$}

We compute push-forwards to $B_{3}$ of intersections in $\tilde{Q}$ as follows. From the fact that $\left(\pi_{B_{3}}\right)_{*}\left(\left(Z_{0}\right) \cdot\left(Z_{23}\right)\right)$ is supported on the curve $\left\{z=a_{5}=0\right\}$ we conclude that

$$
\left(\pi_{B_{3}}\right)_{*}\left(\left(Z_{0}\right) \cdot\left(Z_{23}\right)\right) \equiv 0 .
$$


Appealing to (7.25) and (7.26), we compute

$$
\begin{aligned}
&\left(\pi_{B_{3}}\right)_{*}((X) \cdot \tilde{Q}(X)) \equiv-N \\
&\left(\pi_{B_{3}}\right)_{*}\left((X) \cdot \tilde{Q}\left(Z_{0}\right)\right) \equiv\left(\pi_{B_{3}}\right)_{*}\left((X) \cdot \tilde{Q}\left(Z_{23}\right)\right) \equiv 0 \\
&\left(\pi_{B_{3}}\right)_{*}\left((X) \cdot \tilde{Q} \mathcal{C}_{\text {Higgs }}\right) \equiv 0 \\
&\left(\pi_{B_{3}}\right)_{*}\left(\left(Z_{0}\right)^{2}\right) \\
&\left(\pi_{B_{3}}\right)_{*}\left(\left(Z_{23}\right)^{2}\right) \equiv-N .
\end{aligned}
$$

\section{Higgs line bundle and the $G$-flux}

\subsection{Physical interpretation}

We begin with the projection map

$$
\mathcal{D} \subseteq B_{3} \times \mathbb{P}_{[\tilde{t}, \tilde{z}]} \stackrel{\psi}{\rightarrow} B_{3}
$$

Recall that $E_{8}$ has the subgroup $\mathrm{SU}(5)_{\text {gauge }} \times \mathrm{SU}(4)_{\text {Higgs }} \times \mathrm{U}(1)_{X}$ and the Higgs operator is a non-trivial element of the center of the enveloping algebra of $\mathrm{SU}(4)_{\mathrm{Higgs}}$. We use it to break $E_{8}$-symmetry of the subgroup SU(4) $)_{\text {Higgs }}$. To accomplish this we must identify a line bundle

$$
\mathcal{L}_{\text {Higgs }}
$$

on $\mathcal{D}$ such that the first Chern class of the rank-4 vector bundle

$$
\mathcal{E}:=\psi_{*}\left(\mathcal{L}_{\text {Higgs }}\right)
$$

is zero. We then geometrically specify the eigenvalues of the Higgs operator acting on the complexified Cartan subalgeba of SU(4) Higgs. We identify the fibers of $\mathcal{E}$ with the representation space for the fundamental representation of $\mathrm{SU}(4)$ so that the roots

$$
\frac{t}{z}=\tilde{t}_{1}, \ldots, \tilde{t}_{4}
$$

of

$$
a_{5} t^{4}+a_{54} t^{3} z-a_{20} t^{2} z^{2}-a_{0} z^{3}(t+z)=0
$$

in (3.8) become the eigenvalues of the Higgs operator on the eigenvectors of the standard representation of SU(4) Higgs $_{\text {. The condition }}$

$$
c_{1}(\mathcal{E})=0
$$

becomes the condition that the sum of these eigenvalues is zero. 


\subsection{The Higgs bundle}

So we begin with any line bundle $\mathcal{L}$ on $\mathcal{D}^{(4)}$. By the Grothendieck Riemann-Roch theorem,

$$
c_{1}\left(\psi_{*}(\mathcal{L})\right)=\psi_{*}\left(c_{1}(\mathcal{L})\right)-\psi_{*}\left(c_{1}\left(\mathcal{O}_{\mathcal{D}}\left(\frac{R}{2}\right)\right)\right)
$$

where $R$ is the ramification divisor of $\psi$.

To construct $\mathcal{L}_{\text {Higgs }}$ we proceed as follows. Applying the formula for the discriminant of a fourth-degree equation (7.1) we obtain that the discriminant has class $6 \cdot N$ on $B_{3}$. Thus we must have

$$
\psi_{*}\left(c_{1}\left(\mathcal{O}_{\mathcal{D}}\left(\frac{R}{2}\right)\right)\right)=c_{1}\left(\mathcal{N}^{3}\right)
$$

so that we must choose an effective divisor class on $\mathcal{D}^{(4)}$ whose push-forward to $B_{3}$ has class $c_{1}\left(\mathcal{N}^{3}\right)$.

One obvious line bundle to use is

$$
\left.\mathcal{O}_{B_{3}}(N) \otimes \mathcal{O}_{\mathbb{P}_{[\tilde{t}, \tilde{z}]}}(-1)\right|_{\mathcal{D}}
$$

that pushes forward on $B_{3}$ to

$$
3 N .
$$

We denote the line bundle on $\mathcal{D}^{(4)}$ given by this divisor as

$$
\mathcal{L}_{\text {Higgs }}^{(0)}=\left.\mathcal{O}_{B_{3}}(N) \otimes \mathcal{O}_{\mathbb{P}_{[U, V]}}(-1)\right|_{\mathcal{D}^{(4)}} .
$$

From subsection 7.4 the matter curves are given by

$$
\begin{aligned}
\Sigma_{\mathbf{1 0}}^{(4)} & =\{t / z=\infty\} \cdot \mathcal{D}^{(4)} \\
\Sigma_{\overline{\mathbf{5}}}^{(41)} & =\{t / z=1\} \cdot \mathcal{D}^{(4)}
\end{aligned}
$$

and, as in [15] the Higgs curve is given by a branched double cover $\hat{\Sigma}_{\overline{5}}^{(44)} \subseteq \mathcal{D}^{(4)}$ of the curve

$$
\Sigma_{\overline{\mathbf{5}}}^{(44)}=\left\{z=\left|\begin{array}{cc}
a_{4} & -a_{5} \\
a_{3}+a_{0} & a_{3}
\end{array}\right|=0\right\} \subseteq B_{3} .
$$

We will need the skew component of the push-forward to $\Sigma_{\overline{5}}^{(44)}$ of $\mathcal{L}_{\text {Higgs }}^{(0)}$. Since the skew component of the restriction of $\mathcal{O}_{\mathbb{P}_{[\tilde{t}, \tilde{z}]}}(-1)$ is trivial, we need only compute the skew component of the push-forward of the pull-back of $\mathcal{O}_{B_{3}}(N)$. Again by the Grothendieck Riemann-Roch theorem, this is a line bundle on $\Sigma_{\overline{5}}^{(44)}$ whose square is the restriction of $\mathcal{O}_{B_{3}}(2 N)$. We will denote this line bundle as $\mathcal{L}_{\text {Higgs }}^{(0,-)}$. Since the restriction of $\mathcal{O}_{B_{3}}(3 N)$ to $\Sigma_{\overline{5}}^{(44)}$ is its canonical bundle, the restriction of $\mathcal{L}_{\text {Higgs }}^{(0,-)}$ will be a theta-characteristic of $\Sigma_{\overline{5}}^{(44)}$. 


\subsection{Restrictions to the matter and Higgs curves}

From (4.1) of [15] we have

$$
c_{1}\left(K_{B_{3}}^{-1}\right)^{3}=12
$$

and so

$$
\begin{gathered}
\operatorname{deg}\left(K_{\Sigma_{\mathbf{1 0}}^{(4)}}\right)=N^{3}=12 \\
\operatorname{deg}\left(K_{\Sigma_{\overline{5}}^{(41)}}\right)=N^{3}=12 \\
\operatorname{deg}\left(K_{\Sigma_{\overline{5}}^{(44)}}\right)=4 N^{3}=48 .
\end{gathered}
$$

Since the degree of the canonical bundle of a curve is $2 g-2$, the genus of $\Sigma_{\overline{5}}^{(44)}$ is 25 and the genus of both $\Sigma_{10}^{(4)}$ and $\Sigma_{\overline{5}}^{(41)}$ is 7 .

To compute with $\mathcal{L}_{\text {Higgs }}^{(0)}$ we work on the preimage $\mathcal{D}$ of $\mathcal{C}_{\text {Higgs }}$ in $B_{3} \times \mathbb{P}_{[U, V]}$ where

$$
\mathcal{L}_{\text {Higgs }}^{(0)}=K_{B_{3}}^{-1} \otimes \mathcal{O}_{\mathbb{P}_{[U, V]}}(-1) .
$$

Thus

$$
\left.\mathcal{L}_{\text {Higgs }}^{(0)}\right|_{\Sigma_{\mathbf{1 0}}^{(4)}}=\left.\mathcal{N}\right|_{\Sigma_{\mathbf{1 0}}^{(4)}}=\left.K_{B_{3}}^{-1}\right|_{\Sigma_{\mathbf{1 0}}^{(4)}}=K_{\Sigma_{\mathbf{1 0}}^{(4)}}
$$

and

$$
\left.\mathcal{L}_{\text {Higgs }}^{(0)}\right|_{\Sigma_{\overline{5}}^{(41)}}=\left.\mathcal{N}\right|_{\Sigma_{\overline{5}}^{(41)}}=\left.K_{B_{3}}^{-1}\right|_{\Sigma_{\overline{5}}^{(41)}}=K_{\Sigma_{\overline{5}}^{(41)}}
$$

As we showed just above

$$
\left.\mathcal{L}_{\text {Higgs }}^{(0,-)}\right|_{\Sigma_{\overline{5}}^{(44)}}=\left.\mathcal{N}\right|_{\Sigma_{\overline{5}}^{(44)}}
$$

so that, in particular

$$
\left(\left.\mathcal{L}_{\text {Higgs }}^{(0,-)}\right|_{\Sigma_{\overline{5}}^{(44)}}\right)^{2}=K_{\Sigma_{\overline{5}}^{(44)}}
$$

In fact, $\left.\mathcal{L}_{\text {Higgs }}^{(0)}\right|_{\Sigma_{\overline{5}}^{(44)}}$ is nothing more than the theta-characteristic $Z_{2} \cdot Z$ in section 5.1 of [15] for $Z_{2}=\Sigma_{\overline{5}}^{(44)}$.

Thus

$$
\begin{aligned}
& h^{0}\left(\left.\mathcal{L}_{\text {Higgs }}^{(0)}\right|_{\Sigma_{\text {10 }}^{(4)}}\right)-h^{1}\left(\left.\mathcal{L}_{\text {Higgs }}^{(0)}\right|_{\Sigma_{\text {10 }}^{(4)}}\right)=7-1=6 \\
& h^{0}\left(\left.\mathcal{L}_{\text {Higgs }}^{(0)}\right|_{\Sigma_{\overline{5}}^{(41)}}\right)-h^{1}\left(\left.\mathcal{L}_{\text {Higgs }}^{(0)}\right|_{\Sigma_{\overline{5}}^{(41)}}\right)=7-1=6 \\
& h^{0}\left(\left.\mathcal{L}_{\text {Higgs }}^{(0,-)}\right|_{\Sigma_{\overline{5}}^{(44)}}\right)-h^{1}\left(\left.\mathcal{L}_{\text {Higgs }}^{(0,-)}\right|_{\Sigma_{\overline{5}}^{(44)}}\right)=0 .
\end{aligned}
$$

We therefore have the desired Euler characteristics for the Higgs bundle on the matter and Higgs curves. However the ranks of the relevant spaces of sections are not quite right.

We will rectify the undesired outcome by modifying $\mathcal{L}_{\text {Higgs }}^{(0)}$ by recalling from section 7.1 of $[15]$ that

$$
\left(\left(S_{\mathrm{GUT}} \cap\left(F_{\dot{x} \dot{z} \ddot{y} \ddot{w}} \cup F_{\dot{y} \dot{w} \ddot{x} \ddot{z})}\right) \times \mathbb{P}_{[U, V]}\right) \subseteq \mathcal{D} .\right.
$$


This allows us to define

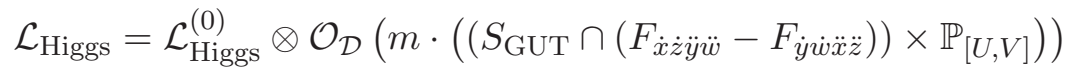

for $m \neq 0$ as in lemma 6 of [15] and define

$$
\begin{aligned}
\mathcal{L}_{\mathbf{1 0}}^{(4)} & :=\left.\mathcal{L}_{\text {Higgs }}\right|_{\Sigma_{\mathbf{1 0}}^{(4)}} \\
\mathcal{L}_{\overline{\mathbf{5}}}^{(41)} & :=\left.\mathcal{L}_{\text {Higgs }}\right|_{\Sigma_{\overline{\mathbf{5}}}^{(41)}} \\
\mathcal{L}_{\overline{\mathbf{5}}}^{(44)} & :=\left.\mathcal{L}_{\text {Higgs }}^{(0,-)}\right|_{\Sigma_{\overline{5}}^{(44)}} .
\end{aligned}
$$

Since the restriction of $\mathcal{O}_{\mathcal{D}}\left(m \cdot\left(\left(S_{\mathrm{GUT}} \cap\left(F_{\dot{x} \dot{z} \ddot{y} \ddot{w}}-F_{\dot{y} \dot{w} \ddot{x} \ddot{z}}\right)\right) \times \mathbb{P}_{[U, V]}\right)\right)$ to the respective curves is not the trivial bundle, we conclude that

$$
\begin{aligned}
& h^{0}\left(\mathcal{L}_{\mathbf{1 0}}^{(4)}\right)=6 \\
& h^{1}\left(\mathcal{L}_{\mathbf{1 0}}^{(4)}\right)=0
\end{aligned}
$$

and

$$
\begin{aligned}
& h^{0}\left(\mathcal{L}_{\overline{\mathbf{5}}}^{(41)}\right)=6 \\
& h^{1}\left(\mathcal{L}_{\overline{\mathbf{5}}}^{(41)}\right)=0 .
\end{aligned}
$$

Furthermore by lemma 7 of [15] we have with respect to the eigenbundles of the involution $C_{u, v}$ that

$$
\begin{aligned}
& h^{0}\left(\left(\pi_{\Sigma_{\overline{5}}^{(44)}}\right)_{*}\left(\mathcal{L}_{\overline{\mathbf{5}}}^{(44)}\right)^{[+1]}\right)=0 \\
& h^{1}\left(\left(\pi_{\Sigma_{\overline{5}}^{(44)}}\right)_{*}\left(\left.\mathcal{L}_{\text {Higgs }}\right|_{\hat{\Sigma}_{\overline{5}}^{(44)}} ^{[-1]}\right)^{[+1]}\right)=0 \\
& h^{0}\left(\left(\pi_{\Sigma_{\overline{5}}^{(44)}}\right)_{*}\left(\left.\mathcal{L}_{\text {Higgs }}\right|_{\hat{\Sigma}_{\overline{5}}^{(44)}} ^{[-1]}\right)^{[-1]}\right)=1 \\
& h^{1}\left(\left(\pi_{\Sigma_{\overline{5}}^{(44)}}\right)_{*}\left(\left.\mathcal{L}_{\mathrm{Higgs}}\right|_{\hat{\Sigma}_{\overline{5}}^{(44)}} ^{[-1]}\right)^{[-1]}\right)=1 .
\end{aligned}
$$

This gives three-generations

$$
\begin{aligned}
& h^{0}\left(\Sigma_{\mathbf{1 0}}^{(4) \vee} ; \mathcal{L}_{\mathbf{1 0}}^{(4)[ \pm 1]}\right)=h^{0}\left(\Sigma_{\overline{\mathbf{5}}}^{(41) \vee} ; \mathcal{L}_{\overline{\mathbf{5}}}^{(41)[ \pm 1]}\right)=3 \\
& h^{1}\left(\Sigma_{\mathbf{1 0}}^{(4) \vee} ; \mathcal{L}_{\mathbf{1 0}}^{(4)[ \pm 1]}\right)=h^{1}\left(\Sigma_{\overline{\mathbf{5}}}^{(41) \vee} ; \mathcal{L}_{\overline{\mathbf{5}}}^{(41)[ \pm 1]}\right)=0
\end{aligned}
$$

as well as

$$
\begin{aligned}
& h^{0}\left(\Sigma_{\overline{\mathbf{5}}}^{(44) \vee} ; \mathcal{L}_{\overline{\mathbf{5}}}^{(44)[+1]}\right)=h^{1}\left(\Sigma_{\overline{\mathbf{5}}}^{(44) \vee} ; \mathcal{L}_{\overline{\mathbf{5}}}^{(44)[+1]}\right)=0 \\
& h^{0}\left(\Sigma_{\overline{\overline{5}}}^{(44) \vee} ; \mathcal{L}_{\overline{5}}^{(44)[-1]}\right)=h^{1}\left(\Sigma_{\overline{5}}^{(44) \vee} ; \mathcal{L}_{\overline{5}}^{(44)[-1]}\right)=1
\end{aligned}
$$

where the sign indicates the $C_{u, v}$-eigen-line-bundle. 


\subsection{Action of $\tilde{\boldsymbol{\beta}}_{4}$ on $\mathcal{L}_{\text {Higgs }}$}

The isomorphisms

$$
(\tilde{\zeta}) \leftrightarrow B_{3} \leftrightarrow(\tilde{\tau})
$$

induced by $\tilde{\beta}_{4}$ and $\pi_{B_{3}}$ identifies sections of $\mathcal{O}_{B_{3}}(N)$ with sections of the co-normal bundles $\mathcal{N}_{(\tilde{\zeta}) \mid \tilde{W}_{4}}^{\vee}$ and $\mathcal{N}_{(\tilde{\tau}) \mid \tilde{W}_{4}}^{\vee}$ respectively. A section of $\mathcal{O}_{B_{3}}(N)$ pulls back to sections of $\mathcal{N}_{(\tilde{\zeta}) \mid \tilde{W}_{4}}^{\vee}$ and $\mathcal{N}_{(\tilde{\tau}) \mid \tilde{W}_{4}}^{\vee}$ respectively that are interchanged under the action of $\tilde{\beta}_{4}$ thereby identifying $\mathcal{N}_{(\tilde{\zeta}) \mid \tilde{W}_{4}}^{\vee}$ with $\tilde{\beta}^{*} \mathcal{N}_{(\tilde{\tau}) \mid \tilde{W}_{4}}^{\vee}$. In (8.4) we have used that identification to define an involution on the rank-2 vector bundle $\left(\pi_{\mathcal{C}_{\text {Higgs }}^{\vee}}\right)_{*} \mathcal{L}_{\text {Higgs }}$ that decomposes into the direct sum of line bundles consisting of sections that respectively symmetric and skew-symmetric. It is the skew-symmetric line bundle on $\mathcal{C}_{\text {Higgs }}^{\vee}$ that we call $\mathcal{L}_{\text {Higgs }}^{\vee}$.

Let

$$
\check{\mathcal{L}}_{\mathbf{1 0}}^{(4)}, \check{\mathcal{L}}_{\overline{\mathbf{5}}}^{(41)}, \check{\mathcal{L}}_{\overline{\mathbf{5}}}^{(44)}
$$

denote the restriction of the line bundle $\mathcal{L}_{\text {Higgs }}^{\vee}$ to the respective quotient curves.

\subsection{The $G$-flux in $\tilde{W}_{4}$}

The $G$-flux $\mathcal{G}_{\tilde{W}_{4}}$ is defined as the push-forward into $\tilde{W}_{4}$ of the Chern class of $\mathcal{L}_{\text {Higgs }}$ modified by a $\tilde{\beta}_{4}$-invariant two cycle in $(X)$ so that it has intersection number zero with $\pi_{B_{3}}^{*}(C)$ for any curve $C$ in $S_{\text {GUT }}$. Since we can essentially identify $\mathcal{C}_{\text {Higgs }} \subseteq \tilde{Q}$ with its lifting $\tilde{\mathcal{C}}_{\text {Higgs }} \subseteq \tilde{W}_{4}$ we will first work to compute the class of the $G$-flux $\mathcal{G}_{\tilde{Q}} \subseteq \tilde{Q}$. By $(7.27)$

$$
\mathcal{C}_{\text {Higgs }}^{(4)} \equiv 4(X)+5 N \in \operatorname{Pic}(\tilde{Q})
$$

Therefore the class of the push-forward of $c_{1}\left(\mathcal{L}_{\text {Higgs }}\right)$ to $\tilde{Q}$ is

$$
(4(X)+5 N) \cdot \tilde{Q}(N+m F) .
$$

Since $F=F_{+}-F_{-}$projects into the curve $\left\{z=\vartheta_{0}+a_{420}=0\right\} \subseteq B_{3}$

$$
\left(\pi_{B_{3}}\right)_{*}(m F)=0
$$

and we have by the projection formula that the intersection of (8.9) with $\pi_{B_{3}}^{*}(C)$ projects to $C \cdot B_{3}(4 N)$, we must have

$$
\mathcal{G}_{\tilde{Q}} \equiv(4 N+5 N) \cdot \tilde{Q}(N+m F)
$$

So

$$
\left(\pi_{B_{3}}\right)_{*}\left(\mathcal{G}_{\tilde{Q}}^{2}\right)=0
$$

and therefore, recalling that $\mathcal{G}_{\tilde{W}_{4}}$ projects birationally to $\mathcal{G}_{\tilde{Q}}$,

$$
\mathcal{G}_{\tilde{Q}}^{2}=\mathcal{G}_{\tilde{W}_{4}}^{2}=0
$$




\subsection{Numerical conditions on the square of the $G$-flux}

The standard conditions that $\mathcal{G}_{W_{4}^{\vee}}$ will have to satisfy are that

$$
\begin{aligned}
\mathcal{G}_{W_{4}^{\vee}} \cdot W_{4}^{\vee} \mathcal{G}_{W_{4}^{\vee}} & \geq 0 \\
\mathcal{G}_{W_{4}^{\vee}} \cdot W_{4}^{\vee} & \mathcal{G}_{W_{4}^{\vee}}-\frac{\chi\left(W_{4}^{\vee}\right)}{24} \leq 0
\end{aligned}
$$

where the subscript on the intersection dot indicates the space in which the intersection is taking place. But by (8.10),

$$
\mathcal{G}_{W_{4}^{\vee}} \cdot W_{4}^{\vee} \mathcal{G}_{W_{4}^{\vee}}=\mathcal{G}_{\tilde{W}_{4}}^{2}=0
$$

so we must only check that $\frac{\chi\left(W_{4}^{\vee}\right)}{24}$ is a non-negative integer.

Now the involution $\tilde{\beta}_{4}$ on $W_{4}$ has four fixpoints over each of the eight fixpoints of the action of the involution $\beta_{3}$ on $B_{3}$. As in formula (5.3) of [43] each orbifold point will affect the computation of $\chi\left(W_{4}^{\vee}\right)$ in (8.11). That is, since the (string-theoretic) orbifold Euler characteristic of the fiber of $\tilde{W}_{4} / B_{3}$ over each fixpoint of $\beta_{3}$ in $B_{3}$ is 6 ,

$$
\chi\left(W_{4}^{\vee}\right)=\frac{1}{2} \chi\left(\tilde{W}_{4}\right)+8 \cdot 6 .
$$

Since $\tilde{W}_{4}$ is smooth with a $(4+1)$-split of $\tilde{\mathcal{C}}_{\text {Higgs }}$ we can apply the formula

$$
\chi\left(\tilde{W}_{4}\right)=6 \int_{B_{3}^{\vee}}\left(24 c_{1}^{3}-44 c_{1}^{2} \cdot N+27 c_{1} \cdot N^{2}-5 N^{3}+2 c_{1} c_{2}\right)
$$

given in (5.19) of [43] where $c_{j}$ denotes the $j$-th Chern class of $B_{3}$. Now in our case $N=c_{1}$ and, since $\chi\left(\mathcal{O}_{B_{3}}\right)=1$, the Riemann-Roch theorem gives that $\int_{B_{3}} c_{1} \cdot c_{2}=24$. Furthermore, by (4.1) of $[15] c_{1}^{3}=12$. So

$$
\chi\left(\tilde{W}_{4}\right)=12 \int_{B_{3}^{\vee}}\left(c_{1}^{3}+c_{1} c_{2}\right)=12(12+24)
$$

and

$$
\chi\left(W_{4}^{\vee}\right)=6 \cdot(36+8)=24 \cdot 11 .
$$

\subsection{The $D$-term}

We next consider the $\mathrm{U}(1)$ factor of the $(4+1)$-decomposition of the spectral variety. Following [24] this $D$ term must vanish, otherwise we might break $R$-parity in the low energy theory.

After resolution,

$$
\tilde{W}_{4}-E_{\overline{5}}^{(44)} \rightarrow \tilde{Q}-C_{\overline{5}}^{(44)}
$$

is a branched double cover, so that $\tilde{W}_{4}$ will inherit ample divisors from sufficiently ample divisors on $\tilde{Q}$. So, to compute the $D$-term we must first adjust the divisor

$$
\tilde{\Delta}-4(X) \equiv 4 N-2\left(\left(Z_{0}\right)+\left(Z_{23}\right)\right)
$$


so that its intersections with all curves of the form $(X) \cdot \tilde{Q} D$ and $\pi_{B_{3}}^{*}$ (point) are zero. Now

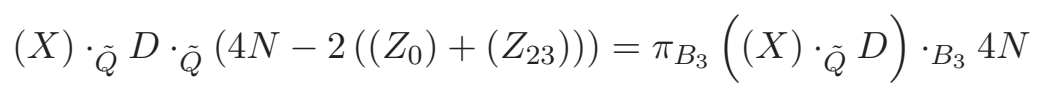

so that we must adjust by $-4 N$. But the $D$-term is computed by identifying an ample divisor on $\tilde{Q}$ that has zero intersection number with the two-class

$$
\mathcal{G}_{\tilde{Q}} \cdot \tilde{Q}\left(\mathcal{C}_{\text {Higgs }}-4(\hat{X})-\hat{S}_{\mathrm{GUT}}\right) .
$$

But

$$
\mathcal{G}_{\tilde{Q}} \cdot \tilde{Q}\left(\mathcal{C}_{\text {Higgs }}-4(\hat{X})-\hat{S}_{\mathrm{GUT}}\right)=\mathcal{G}_{\tilde{Q}} \cdot \tilde{Q}^{0}=0
$$

so any ample divisor trivially has zero intersection with (8.12).

\section{Wilson line: symmetry-breaking to the standard model}

We write

$$
\left(\pi_{B_{3}^{\vee}}\right)_{*} \mathcal{O}_{B_{3}}=\mathcal{O}_{B_{3}^{\vee}} \oplus \mathcal{O}_{B_{3}}\left(\varepsilon_{u, v}\right)
$$

with connection isomorphism

$$
\varepsilon_{u, v}: \pi_{1}\left(B_{3}^{\vee}\right) \rightarrow\{ \pm 1, \cdot\}
$$

Let

$$
Y=\left(\begin{array}{rrrr}
-1 / 3 & & & \\
& -1 / 3 & & \\
& -1 / 3 & \\
& & 1 / 2 & \\
& & & 1 / 2
\end{array}\right) \in \mathfrak{s u}(5)
$$

denote the hypercharge direction when acting on the fundamental represesentation. The Wilson line is the flat rank-5 vector bundle $L_{Y}$ given by the homomorphism

$$
\pi_{1}\left(S_{\mathrm{GUT}}^{\vee}\right) \rightarrow \mathrm{SU}(5)_{\text {gauge }}
$$

that takes the generator to $\exp (6 \pi i \cdot Y)$ when viewed as acting on the fundamental representation of $\mathrm{SU}(5)_{\text {gauge. }}$ This has the effect of breaking $\mathrm{SU}(5)_{\text {gauge-representations }}$ over $S_{\mathrm{GUT}}$ to $\mathrm{SU}(3) \times \mathrm{SU}(2) \times \mathrm{U}(1)_{Y}$-representations over $S_{\mathrm{GUT}}^{\vee}$. Via tensor and exterior product, these representations yield eigenspaces respect to the $\mathbb{Z}_{2} \times \mathbb{Z}_{2}=\{ \pm 1\} \times$ $\{\exp (6 \pi i \cdot Y)\}$ holonomy group. Only eigenspaces with eigenvalue pairs in the diagonal of $\mathbb{Z}_{2} \times \mathbb{Z}_{2}$ remain in the theory. The flat $\mathrm{U}(1)_{Y}$-bundle gives mass to the gauge states in $\mathrm{SU}(5) /\left[\mathrm{SU}(3) \times \mathrm{SU}(2) \times \mathrm{U}_{Y}(1)\right]$ at a scale given by the cycle $\propto 1 / R_{\text {cycle }}$ on the GUT surface. ${ }^{5}$ It endows the quotient

$$
W_{4}^{\vee} / B_{3}^{\vee}
$$

with the Standard Model gauge symmetry $\mathrm{SU}(3) \times \mathrm{SU}(2) \times \mathrm{U}(1)_{Y}$.

\footnotetext{
${ }^{5}$ Referring to lemma 2 the flat $\mathrm{U}(1)_{Y}$-bundle wraps the non-contractible cycle $(v)$ while $(\zeta)$ and $(\tau)$ are interchanged.
} 
We are about to incorporate the very particular translation by $\tau\left(b_{3}\right)-\zeta\left(b_{3}\right)$ in the fiber direction into the definition of the $\mathbb{Z}_{2}$-action on $\tilde{W}_{4} / B_{3}$. This translation commutes with all the properties we have attributed to $\tilde{\beta}_{4} / \beta_{3}$ up to this point. The purpose of including the change of basepoint into the involution $\tilde{\beta}_{4} / \beta_{3}$ is to eliminate vector-like exotics from the bulk spectrum. If we were not to incorporate the translation $\tau\left(b_{3}\right)-\zeta\left(b_{3}\right)$ into $\tilde{\beta}_{4} / \beta_{3}$, the bulk spectrum data for $\mathrm{SU}(3) \times \mathrm{SU}(2) \times \mathrm{U}(1)_{Y}$ would be detailed in the following table, in which the non-vanishing of the cohomology groups in the last two rows indicate the existence of vector-like exotics [6, 20,43]:

\begin{tabular}{|c|c|c|}
\hline Representation & Type of multiplet & Cohomology group dimension \\
\hline$(\mathbf{8}, \mathbf{1})_{0}$ & Vector & $h^{2}\left(S_{\mathrm{GUT}}^{\vee}, K_{S_{\mathrm{GUT}}^{\vee}}\right)=1$ \\
\hline$(\mathbf{1}, \mathbf{3})_{0}$ & Vector & $h^{2}\left(S_{\mathrm{GUT}}^{\vee}, K_{S_{\mathrm{GUT}}^{\vee}}\right)=1$ \\
\hline$(\mathbf{1}, \mathbf{1})_{0}$ & Vector & $h^{2}\left(S_{\mathrm{GUT}}^{\vee}, K_{S_{\mathrm{GUT}}^{\vee}}\right)=1$ \\
\hline$(\mathbf{8}, \mathbf{1})_{0}$ & Chiral & $h^{0}\left(S_{\mathrm{GUT}}^{\vee}, K_{S_{\mathrm{GUT}}^{\vee}}^{\vee}\right) \oplus h^{1}\left(S_{\mathrm{GUT}}^{\vee}, K_{S_{\mathrm{GUT}}^{\vee}}\right)=0$ \\
\hline$(\mathbf{1}, \mathbf{3})_{0}$ & Chiral & $h^{0}\left(S_{\mathrm{GUT}}^{\vee}, K_{S_{\mathrm{GUT}}^{\vee}}\right) \oplus h^{1}\left(S_{\mathrm{GUT}}^{\vee}, K_{S_{\mathrm{GUT}}^{\vee}}\right)=0$ \\
\hline$(\mathbf{1}, \mathbf{1})_{0}$ & Chiral & $h^{0}\left(S_{\mathrm{GUT}}^{\vee}, K_{S_{\mathrm{GUT}}^{\vee}}\right) \oplus h^{1}\left(S_{\mathrm{GUT}}^{\vee}, K_{S_{\mathrm{GUT}}^{\vee}}\right)=0$ \\
\hline$(\mathbf{3}, \mathbf{2})_{-5 / 6}$ & Vector & $h^{0}\left(S_{\mathrm{GUT}}^{\vee}, \mathcal{O}_{S_{\mathrm{GUT}}^{\vee}}\left(\varepsilon_{u, v}\right)\right)=0$ \\
\hline$(\overline{\mathbf{3}}, \mathbf{2})_{5 / 6}$ & Vector & $h^{0}\left(S_{\mathrm{GUT}}^{\vee}, \mathcal{O}_{S_{\mathrm{GUT}}^{\vee}}\left(\varepsilon_{u, v}\right)\right)=0$ \\
\hline$(\mathbf{3}, \mathbf{2})_{-5 / 6}$ & Chiral & $h^{1}\left(S_{\mathrm{GUT}}^{\vee}, \mathcal{O}_{S_{\mathrm{GUT}}^{\vee}}\left(\varepsilon_{u, v}\right)\right) \oplus h^{2}\left(S_{\mathrm{GUT}}^{\vee}, \mathcal{O}_{S_{\mathrm{GUT}}^{\vee}}\left(\varepsilon_{u, v}\right)\right)=1$ \\
\hline$(\overline{\mathbf{3}}, \mathbf{2})_{5 / 6}$ & Chiral & $h^{1}\left(S_{\mathrm{GUT}}^{\vee}, \mathcal{O}_{S_{\mathrm{GUT}}^{\vee}}\left(\varepsilon_{u, v}\right)\right) \oplus h^{2}\left(S_{\mathrm{GUT}}^{\vee}, \mathcal{O}_{S_{\mathrm{GUT}}}\left(\varepsilon_{u, v}\right)\right)=1$ \\
\hline
\end{tabular}

Reviewing the construction of the 'semi-stable degeneration' in section 5 one sees that all the modification steps

$$
\begin{array}{rrrr}
\bar{W}_{4}^{\wedge} / B_{3}^{\wedge} & \rightarrow & \bar{V}_{3}^{\wedge} / B_{2} \subseteq & \overline{d P}_{a} \cup \overline{d P}_{b} \\
\downarrow & \downarrow & \\
\bar{W}_{4} / B_{3} & \rightarrow & \bar{V}_{3} / B_{2} \subseteq & \overline{d P}_{a} \cup \overline{d P}_{b} \\
\uparrow & \uparrow & \uparrow \\
W_{4}^{(1)} / B_{3} & \rightarrow & V_{3}^{(1)} / B_{2} \subseteq & d P_{a}^{(1)} \cup d P_{b}^{(1)} \\
\uparrow & \uparrow & \uparrow \\
W_{4}^{(2)} / B_{3} & \rightarrow & V_{3}^{(2)} / B_{2} \subseteq & d P_{a}^{(2)} \cup d P_{b}^{(2)} \\
\uparrow & \uparrow & \\
\tilde{W}_{4} / B_{3} & \rightarrow & V_{3} / B_{2} \subseteq & d P_{a} \cup d P_{b}
\end{array}
$$

are equivariant with respect to the action of the automorphism group of $B_{3}$ given by the 
permutation group $S_{4} \subseteq W \mathrm{SU}(5)$ as in [15]. Now on the Heterotic side $\overline{d P}_{a} \cup \overline{d P}_{b}$ is simply the blow-up of $W_{4,0}$ with center $(v)$.

Since

$$
(x, y) \mapsto(x,-y)
$$

before we introduce the translation into the definition of $\tilde{\beta}_{4}$ we have that

$$
\begin{aligned}
& y\left(\zeta\left(\beta_{3}\left(b_{3}\right)\right)\right)=-y\left(\zeta\left(b_{3}\right)\right) \\
& y\left(\tau\left(\beta_{3}\left(b_{3}\right)\right)\right)=-y\left(\tau\left(b_{3}\right)\right)
\end{aligned}
$$

whereas composing with the translation we have that

$$
\begin{aligned}
& y\left(\zeta\left(\beta_{3}\left(b_{3}\right)\right)\right)=-y\left(\tau\left(b_{3}\right)\right) \\
& y\left(\tau\left(\beta_{3}\left(b_{3}\right)\right)\right)=-y\left(\zeta\left(b_{3}\right)\right)
\end{aligned}
$$

so that

$$
y\left(\tau\left(\beta_{3}\left(b_{3}\right)\right)\right)-y\left(\zeta\left(\beta_{3}\left(b_{3}\right)\right)\right)=-y\left(\zeta\left(b_{3}\right)\right)-\left(-y\left(\tau\left(b_{3}\right)\right)\right)
$$

allowing the translation to descend to the quotient.

Previous construction utilized a single section that was invariant under the involution, thereby forcing the existence of vector-like exotics. The existence of two sections and the incorporation of the translation between them into the $\mathbb{Z}_{2}$-action allows the elimination of the vector-like exotics.

It is $(v)$ on which the non-contractible cycle of $d P_{a} \cup d P_{b}$ is wrapped, while $(\zeta)$ and $(\tau)$ are interchanged. Thus it is $(v)$ that endows the quotient of the action by $\tilde{\beta}_{4}$ with a $\mathrm{U}(1)_{X}$-symmetry.

\section{Bulk and chiral spectra with Wilson Line on $W_{4}^{\vee}$}

\subsection{Bulk spectrum with Wilson line}

Since the canonical bundle of $S_{\mathrm{GUT}}$ is trivial, the introduction of the translation into the definition of $\tilde{\beta}_{4}$ will allow us to replace the trivial line bundle by

$$
\mathcal{O}_{\tilde{W}_{4}}((\tilde{\tau})-(\tilde{\zeta})) \in \operatorname{Pic}\left(\tilde{W}_{4}\right)
$$

in the computation of the bulk spectrum. More specifically, the semi-stable degeneration is the geometric bridge between Heterotic theory and $F$-theory. The introduction of the translation into the definition of $\tilde{\beta}_{4,0}$ affects the dictionary (4.18) that is given in terms of the Weierstrass form on the $d P_{9}$-bundles coming from the $F$-theory side and flat line bundles on the elliptic fibers on the Heterotic side. Change of basepoint from the one given by $\zeta$ to the one given by $\tau$ does not change the uniquely given Weierstrass form of the elliptic fiber however on the other side it does change the sum of eight flat line bundles given by the restriction of the $E_{8}$-bundle since they are given by the differences between each of the eight points marked by the (asymptotic) Tate form and the identity element of the elliptic fiber as a group and the involution changes this last from the intersection of the torus fiber with $(\zeta)$ to the intersection of the torus fiber with $(\tau)$. 
Therefore instead of pushing forward the canonical bundle $K_{S_{\mathrm{GUT}}}=\mathcal{O}_{S_{\mathrm{GUT}}}$ to $S_{\mathrm{GUT}}^{\vee}$ we must incorporate the twist by the bundle (10.1) in order to compute the bulk spectrum for $W_{4}^{\vee} / B_{3}^{\vee}$, that is, we must compute the (derived) cohomology of the push-forward of the twisted structure sheaf

$$
\left(\mathcal{O}_{S_{\mathrm{GUT}} \times_{\hat{B}_{3}} \tilde{W}_{4}}\right) \otimes \mathcal{O}_{\tilde{W}_{4}}((\tilde{\zeta})-(\tilde{\tau}))
$$

that goes to minus itself under the isomorphism $\tilde{\beta}_{4}$. The push-forward of the line bundle (10.1) to $S_{\mathrm{GUT}}^{\vee} \times_{B_{3}} W_{4}^{\vee}$ breaks into the sum

$$
\left(\pi^{*} K_{S_{\mathrm{GUT}}^{\vee}} \otimes \mathcal{O}_{S_{\mathrm{GUT}}^{\vee} \times_{B_{3}} W_{4}^{\vee}}\left(\varepsilon_{u, v}\right)\right) \oplus\left(\mathcal{O}_{S_{\mathrm{GUT}}^{\vee} \times_{B_{3}} W_{4}^{\vee}}\left(\varepsilon_{u, v} \cdot \tilde{\tau}-\varepsilon_{u, v} \cdot \tilde{\zeta}\right)\right)
$$

of symmetric and a skew-symmetric line bundle summands where $\varepsilon_{u, v}$ is the flat orbifold line bundle induced by the involution $\tilde{\beta}_{4}$ with finite fixpoint set.

Thus the derived push-forwards of the summands in (10.2) are respectively

$$
\mathcal{O}_{S_{\text {GUT }}^{\vee}} \oplus\{0\}
$$

are all zero since the divisor $((\tilde{\tau})-(\tilde{\zeta}))$ is not linearly equivalent to zero anywhere, in particular nowhere over $S_{\mathrm{GUT}}$. Therefore referring to the table in section 9 but now with the incorporation of the translation $(\tilde{\tau})-(\tilde{\zeta})$ bulk spectrum data is detailed in the following table:

\begin{tabular}{|c|c|c|}
\hline Representation & Type of multiplet & Cohomology group dimension \\
\hline$(\mathbf{8}, \mathbf{1})_{0}$ & Vector & $h^{2}\left(S_{\mathrm{GUT}}^{\vee}, K_{S_{\mathrm{GUT}}^{\vee}}\right)=h^{0}\left(\mathcal{O}_{S_{\mathrm{GUT}}^{\vee}}\right)=1$ \\
\hline$(\mathbf{1}, \mathbf{3})_{0}$ & Vector & $h^{2}\left(S_{\mathrm{GUT}}^{\vee}, K_{S_{\mathrm{GUT}}^{\vee}}\right)=h^{0}\left(\mathcal{O}_{S_{\mathrm{GUT}}^{\vee}}\right)=1$ \\
\hline$(\mathbf{1}, \mathbf{1})_{0}$ & Vector & $h^{2}\left(S_{\mathrm{GUT}}^{\vee}, K_{S_{\mathrm{GUT}}^{\vee}}\right)=h^{0}\left(\mathcal{O}_{S_{\mathrm{GUT}}^{\vee}}\right)=1$ \\
\hline$(\mathbf{8}, \mathbf{1})_{0}$ & Chiral & $h^{0}\left(S_{\mathrm{GUT}}^{\vee}, K_{S_{\mathrm{GUT}}^{\vee}}\right) \oplus h^{1}\left(S_{\mathrm{GUT}}^{\vee}, K_{S_{\mathrm{GUT}}^{\vee}}\right)=0$ \\
\hline$(\mathbf{1}, \mathbf{3})_{0}$ & Chiral & $h^{0}\left(S_{\mathrm{GUT}}^{\vee}, K_{S_{\mathrm{GUT}}^{\vee}}\right) \oplus h^{1}\left(S_{\mathrm{GUT}}^{\vee}, K_{S_{\mathrm{GUT}}^{\vee}}\right)=0$ \\
\hline$(\mathbf{1}, \mathbf{1})_{0}$ & Chiral & $h^{0}\left(S_{\mathrm{GUT}}^{\vee}, K_{S_{\mathrm{GUT}}^{\vee}}\right) \oplus h^{1}\left(S_{\mathrm{GUT}}^{\vee}, K_{S_{\mathrm{GUT}}^{\vee}}\right)=0$ \\
\hline$(\mathbf{3}, \mathbf{2})_{-5 / 6}$ & Vector & $h^{0}\left(\mathcal{O}_{S_{\mathrm{GUT}}^{\vee}} \times_{B_{3}^{\vee}} W_{4}^{\vee}\left(\varepsilon_{u, v} \cdot \tilde{\tau}-\varepsilon_{u, v} \cdot \tilde{\zeta}\right)\right)=0$ \\
\hline$(\overline{\mathbf{3}}, \mathbf{2})_{5 / 6}$ & Vector & $h^{0}\left(\mathcal{O}_{S_{\mathrm{GUT}}^{\vee} \times B_{3}^{\vee} W_{4}^{\vee}}\left(\varepsilon_{u, v} \cdot \tilde{\tau}-\varepsilon_{u, v} \cdot \tilde{\zeta}\right)\right)=0$ \\
\hline$(\mathbf{3}, \mathbf{2})_{-5 / 6}$ & Chiral & $h^{1}\left(\mathcal{O}_{S_{\mathrm{GUT}}^{\vee} \times_{B_{3}^{\vee}} W_{4}^{\vee}}\left(\varepsilon_{u, v} \cdot \tilde{\tau}-\varepsilon_{u, v} \cdot \tilde{\zeta}\right)\right) \oplus h^{2}(\ldots)=0$ \\
\hline$(\overline{\mathbf{3}, 2})_{5 / 6}$ & Chiral & $h^{1}\left(\mathcal{O}_{S_{\mathrm{GUT}}^{\vee} \times_{B_{3}} W_{4}^{\vee}}\left(\varepsilon_{u, v} \cdot \tilde{\tau}-\varepsilon_{u, v} \cdot \tilde{\zeta}\right)\right) \oplus h^{2}(\ldots)=0$ \\
\hline
\end{tabular}

Thus there are no vector-like exotics! 


\subsection{Matter spectrum}

In fact we have already computed the correct chiral spectra in (8.2), (8.3), and (8.4) of subsection 8.3.

Lemma 6. With respect to the $C_{u, v}$-eigen-line-bundle decomposition of the push-forwards to $B_{3}^{\vee}$ of the (restrictions of the) Higgs bundle to the matter and Higgs curves in $B_{3}$,

i)

$$
\begin{aligned}
& h^{0}\left(\check{\mathcal{L}}_{\mathbf{1 0}}^{(4)[ \pm 1]}\right)=h^{0}\left(\check{\mathcal{L}}_{\overline{\mathbf{5}}}^{(41)[ \pm 1]}\right)=3 \\
& h^{1}\left(\check{\mathcal{L}}_{\mathbf{1 0}}^{(4)[ \pm 1]}\right)=h^{1}\left(\check{\mathcal{L}}_{\overline{\mathbf{5}}}^{(41)[ \pm 1]}\right)=0 .
\end{aligned}
$$

ii)

$$
\begin{aligned}
& h^{0}\left(\check{\mathcal{L}}_{\overline{\mathbf{5}}}^{(44)[+1]}\right)=h^{1}\left(\check{\mathcal{L}}_{\overline{\mathbf{5}}}^{(44)[+1]}\right)=0 \\
& h^{0}\left(\check{\mathcal{L}}_{\overline{\mathbf{5}}}^{(44)[-1]}\right)=h^{1}\left(\check{\mathcal{L}}_{\overline{\mathbf{5}}}^{(44)[-1]}\right)=1 .
\end{aligned}
$$

Therefore referring to tables 1 and 2 in section 7 of [15], the flux distribution associated to the $\mathbb{Z}_{2}$-action given by the involution $\tilde{\beta}_{4}$ and the Wilson line that is wrapped by that involution is presented in the tables below. Namely the distribution of MSSM matter fields is as follows:

\begin{tabular}{|c|c|c|c|c|}
\hline$\Sigma_{\mathbf{1 0}}^{(4)}=\left\{a_{5}=z=0\right\}$ & $C_{u, v}$ & $L_{Y}$ & $\mathcal{L}_{\text {Higgs }}$ & $\mathrm{SU}(3) \times \mathrm{SU}(2) \times \mathrm{U}(1)_{Y}$ \\
\hline$h^{0}\left(\check{\mathcal{L}}_{\mathbf{1 0}}^{(4)[ \pm 1]}\right)$ & +1 & +1 & 3 & $(\mathbf{1}, \mathbf{1})_{+1}$ \\
\hline & -1 & -1 & & $(\mathbf{3}, \mathbf{2})_{+1 / 6}$ \\
\hline & +1 & +1 & & $(\overline{\mathbf{3}}, \mathbf{1})_{-2 / 3}$ \\
\hline$h^{1}\left(\check{\mathcal{L}}_{\mathbf{1 0}}^{(4)[ \pm 1]}\right)$ & +1 & +1 & 0 & $(\mathbf{1}, \mathbf{1})_{+1}$ \\
\hline & -1 & -1 & & $(\overline{\mathbf{3}}, \mathbf{2})_{+1 / 6}$ \\
\hline & +1 & +1 & & $(\mathbf{3}, \mathbf{1})_{+2 / 3}$ \\
\hline
\end{tabular}

\begin{tabular}{|c|c|c|c|c|}
\hline$\Sigma_{\overline{\mathbf{5}}}^{(41)}=\left\{a_{420}=z=0\right\}$ & $C_{u, v}$ & $L_{Y}$ & $\mathcal{L}_{\text {Higgs }}$ & $\mathrm{SU}(3) \times \mathrm{SU}(2) \times \mathrm{U}(1)_{Y}$ \\
\hline$h^{0}\left(\check{\mathcal{L}}_{\overline{\mathbf{5}}}^{(41)[ \pm 1]}\right)$ & +1 & +1 & 3 & $(\overline{\mathbf{3}}, \mathbf{1})_{+1 / 3}$ \\
\hline & -1 & -1 & & $(\mathbf{1}, \mathbf{2})_{-1 / 2}$ \\
\hline$h^{1}\left(\check{\mathcal{L}}_{\overline{\mathbf{5}}}^{(41)[ \pm 1]}\right)$ & +1 & +1 & 0 & $(\mathbf{3}, \mathbf{1})_{-1 / 3}$ \\
\hline & -1 & -1 & & $(\mathbf{1}, \mathbf{2})_{+1 / 2}$ \\
\hline
\end{tabular}


As for the Higgs fields we have the following:

\begin{tabular}{|c|c|c|c|c|}
\hline$\Sigma_{\overline{\mathbf{5}}}^{(44)}=\left\{a_{4} a_{3}+a_{5}\left(a_{0}-a_{3}\right)=z=0\right\}$ & $C_{u, v}$ & $L_{Y}$ & $\mathcal{L}_{\mathrm{Higgs}}$ & $\mathrm{SU}(3) \times \mathrm{SU}(2) \times \mathrm{U}(1)_{Y}$ \\
\hline$h^{0}\left(\check{\mathcal{L}}_{\overline{\mathbf{5}}}^{(44)[+1]}\right)$ & +1 & +1 & 0 & $(\overline{\mathbf{3}}, \mathbf{1})_{+1 / 3}$ \\
\hline$h^{0}\left(\check{\mathcal{L}}_{\overline{\mathbf{5}}}^{(44)[-1]}\right)$ & -1 & -1 & 1 & $(\mathbf{1}, \mathbf{2})_{-1 / 2}$ \\
\hline$h^{1}\left(\check{\mathcal{L}}_{\overline{\mathbf{5}}}^{(44)[+1]}\right)$ & +1 & +1 & 0 & $(\mathbf{3}, \mathbf{1})_{-1 / 3}$ \\
\hline$h^{1}\left(\check{\mathcal{L}}_{\overline{\mathbf{5}}}^{(44)[-1]}\right)$ & -1 & -1 & 1 & $(\mathbf{1}, \mathbf{2})_{+1 / 2}$ \\
\hline
\end{tabular}

In conclusion we have reproduced the spectrum of the minimal supersymmetric Standard Model.

\section{Asymptotic $\mathbb{Z}_{4}$ R-symmetry}

The asymptotic $\mathbb{Z}_{4}$-symmetry $T_{u, v}$ of the semi-stable $F$-theory limit constructed on $B_{3}^{\vee}$ in section 6 of [15] acts on sections and twisted sections of the anti-canonical bundle $B_{3}^{\vee}$. Referring to the results and notation in sections 5 and 6 of [15], the asympotic $\mathbb{Z}_{4} \mathbf{R}$ symmetry $T_{u, v}$ constructed there on

$$
\lim _{\delta \rightarrow 0} \tilde{W}_{4, \delta}
$$

is compatible with the following table from subsection 8.2 of $[15]:^{6}$

\begin{tabular}{|c|c|c|}
\hline TABLE 3: $\quad T_{u, v}$ & $T_{u, v}$-charge & space \\
\hline matter fields on $\frac{\Sigma_{10}^{(4)}}{\left\{C_{u, v}\right\}}$ & -1 & $H^{0}\left(\frac{\Sigma_{10}^{(4)}}{\left\{C_{u, v}\right\}} ; \mathcal{L}_{\mathrm{Higgs}}^{\vee,[ \pm 1]}\right)$ \\
\hline matter fields on $\frac{\Sigma_{5}^{(41)}}{\left\{C_{u, v}\right\}}$ & -1 & $H^{0}\left(\frac{\Sigma_{\overline{5}}^{(41)}}{\left\{C_{u, v}\right\}} ; \mathcal{L}_{\text {Higgs }}^{\vee,[ \pm 1]}\right)$ \\
\hline Higgs fields on $\frac{\Sigma_{\overline{5}}^{(44)}}{\left\{C_{u, v}\right\}}$ & $+i$ & $H^{0}\left(\frac{\Sigma_{5}^{(44)}}{\left\{C_{u, v}\right\}} ; \mathcal{L}_{\mathrm{Higgs}}^{\vee,[-1]}\right) / H^{1}\left(\frac{\Sigma_{5}^{(44)}}{\left\{C_{u, v}\right\}} ; \mathcal{L}_{\mathrm{Higgs}}^{\vee,[-1]}\right)$ \\
\hline bulk matter on $\frac{S_{\mathrm{GUT}}}{\left\{C_{u, v}\right\}}$ & $-i$ & $H^{2}\left(K_{\frac{S_{\mathrm{GUT}}}{\left\{C_{u, v}\right\}}}\right)$ \\
\hline
\end{tabular}

The $\mathbb{Z}_{4} \mathrm{R}$ symmetry forbids the Higgs $\mu$-term and dimension 4 and 5 baryon and lepton number violating operators.

${ }^{6}$ Given the $\mathbb{Z}_{4}$ R-charges, $i^{q_{f}+1}$, for the fermionic components of $N=1$ superfields, then the bosonic components of the chiral superfields are given by $i^{q_{f}}$. This is in accord with the anti-commuting superspace coordinate transforming as $\theta^{\prime}=i^{-1} \theta$. Otherwise, these are equivalent to the $\mathbb{Z}_{4} \mathrm{R}$-charges of [39, 40]. 


\section{Conclusions}

In this paper we have constructed an SU(5) GUT F-theory model. We have shown how to break the GUT group with a non-local Wilson line. Thus we are able to identify the GUT scale with the compactification scale of the GUT surface. Our model includes three families of quarks and leptons and one pair of Higgs doublets. The price to pay for this result is that we have a mirror world where the mass scales and couplings of the mirror states may be different than for the MSSM. This mirror world can, in principle, be the dark matter of the universe. There may or may not also be direct couplings of the mirror and MSSM sectors of the theory. These give interesting physics as in $[4,7,13]$. There are no vector-like exotics in the bulk spectrum or on the matter curves, neither are there chiral exotics.

The existence of the $\mathbb{Z}_{4} \mathbf{R}$-symmetry generated by the automorphism $T_{u, v}$ that reverses the sign on the holomorphic 4 -form addresses the issue of dimension-5 proton decay operators and forbids a $\mu$-term [39]. The charges of the matter states under the $\mathbb{Z}_{4} \mathbf{R}$-symmetry are given in table 3. Finally, Wilson line symmetry-breaking is addressed in sections 9 and 10 .

However there are several issues which are not resolved in this paper. Moduli stabilization is not addressed. We have not generated a $\mu$-term. In principle the $\mathbb{Z}_{4} \mathbf{R}$-symmetry can be broken by non-perturbative physics down to matter parity which then allows for a $\mu$-term of order the weak scale and severely suppressed dimension- 5 proton decay operators. We have not discussed the possible Yukawa interactions needed to give quarks and leptons mass. We may or may not also have right-handed neutrinos which would be useful for a see-saw mechanism of neutrino masses. Finally, the $\mathrm{U}(1)_{X}$ gauge symmetry may be broken to $\mathbb{Z}_{2}$ matter parity via non-perturbative effects at the GUT scale or by a Stueckelberg mechanism. This would then allow for right-handed neutrino Majorana masses near the GUT scale.

\section{Acknowledgments}

The authors would like to thank Dave Morrison, Tony Pantev and Sakura Schäfer-Nameki for their guidance and many helpful conversations over several years. The authors would also like to thank Sakura Schäfer-Nameki for her contribution of the appendix to this paper. However the authors themselves take sole responsibility for any errors or omissions in this paper. S.R. acknowledges partial support from DOE grant DE-SC0011726.

\section{A $\quad \mathbb{P}^{112}$ Formulations by Sakura Schäfer-Nameki}

\section{A.1 Realizing $\bar{W}_{4}$ in $\mathbb{P}^{112}$}

An alternative formulation of the elliptic fibrations $\bar{W}_{4}$ can be given in terms of the $\mathbb{P}^{112}$ fibration in [45]. Let

$$
\mathbb{P}^{112}:=\mathbb{P}\left(\mathcal{O}_{B_{3}} \oplus \mathcal{O}_{B_{3}}(2) \oplus \mathcal{O}_{B_{3}}(4)\right)
$$


with projective coordinates $[w, x, y]$. For $c_{i} \in H^{0}\left(\mathcal{O}_{B_{3}}(8-2 i)\right)$ define

$$
\begin{gathered}
c_{0} w^{4}+c_{1} w^{3} x+c_{2} w^{2} x^{2}+c_{3} w x^{3} \\
=y^{2}+\left(b_{0} x^{2}+b_{1} w x+b_{2} w^{2}\right) y .
\end{gathered}
$$

This elliptic fibration has two rational sections

$$
\begin{aligned}
& w=y=0 \\
& w=y+b_{0} x^{2}=0
\end{aligned}
$$

that can be made symmetric by the shift

$$
\begin{aligned}
& y \mapsto y-\frac{b_{0} x^{2}}{2} . \\
& \left|\begin{array}{cc}
h_{0} & -\hat{t}\left(\hat{h}_{1} \hat{z}-\hat{h}_{2} \hat{t}\right) \\
\hat{t}\left(d_{2} \hat{z}+d_{3} \hat{t}\right) & \hat{c}_{4} \hat{t}^{2}\left(\hat{t}^{2}+\hat{z}^{2}\right)+\hat{c}_{3} \hat{t}^{3} \hat{z}+\hat{c}_{1} z^{3}(\hat{t}-\hat{z})
\end{array}\right|=0 . \\
& \left|\begin{array}{cc}
h_{0} & -\hat{t}\left(\hat{h}_{1} \hat{z}-\hat{h}_{2} \hat{t}\right) \\
\hat{t}\left(d_{2} \hat{z}+d_{3} \hat{t}\right) & \hat{c}_{4} \hat{t}^{2}\left(\hat{t}^{2}+\hat{z}^{2}\right)+\hat{c}_{3} \hat{t}^{3} \hat{z}+\hat{c}_{1} z^{3}(\hat{t}-\hat{z})
\end{array}\right|=0 .
\end{aligned}
$$

Denote by

$$
\begin{aligned}
\gamma_{i} & =\operatorname{ord}_{z}\left(c_{i}\right) \\
\beta_{j} & =\operatorname{ord}_{z}\left(b_{j}\right)
\end{aligned}
$$

and let

$$
Q\left(\gamma_{0}, \gamma_{1}, \gamma_{3}, \gamma_{0}, \beta_{0}, \beta_{1}, \beta_{2}\right)
$$

denote the quartic (A.1) for the given values. Using Tate's algorithm, there are various ways to degenerate this to an $I_{5}$ fiber about the locus $\{z=0\}$ [34], both by specifying the vanishing orders in (A.1) without further relations among the leading order coefficients $c_{i}$ and $b_{j}$ ('canonical Tate models'), or by imposing relations among the coefficients ('noncanonical models').

In particular, the fibration $\bar{W}_{4}$ in this language is a non-canonical model with coordinates $(w, x, y)$ given by

$$
\begin{aligned}
X_{0} & \leftrightarrow w \\
Y_{0} & =x \\
W_{0} & \leftrightarrow y+x^{2}
\end{aligned}
$$

so that (6.8) becomes the $Q(2,1,0,0,0, \infty, \infty)$ given in $(6.11)$ by

$$
\begin{aligned}
y^{2}+2 x^{2} y= & -2 a_{5} x^{3} w \\
& +\left(a_{5}^{2}-2 a_{4} z-6 z^{2}\right) x^{2} w^{2} \\
& +\left(2 a_{5} a_{4} z+\left(6 a_{5}-4 a_{420}\right) z^{2}-8 z^{3}\right) x w^{3} \\
& -\left(\left(-a_{4}^{2}-4 a_{5} a_{420}\right) z^{2}+\left(2 a_{4}+4 a_{2}-4 a_{5}\right) z^{3}+3 z^{4}\right) w^{4} .
\end{aligned}
$$




\begin{tabular}{|c|c|c|}
\hline Matter loci & Rep and U(1) charge & Codim 2 fiber \\
\hline$\sigma_{3}$ & $\mathbf{1 0}_{1}+\overline{\mathbf{1 0}}_{-1}$ & $I_{1}^{*(0|| 1)}$ \\
$\sigma_{1}$ & $\mathbf{1 0}_{-4}+\overline{\mathbf{1 0}}_{4}$ & $I_{1}^{*(01)}$ \\
$\sigma_{2}$ & $\mathbf{5}_{-7}+\overline{\mathbf{5}}_{7}$ & $I_{6}^{(0 \mid 1)}$ \\
$P_{1}$ & $\mathbf{5}_{-2}+\overline{\mathbf{5}}_{2}$ & $I_{6}^{(0|| 1)}$ \\
$P_{2}$ & $\mathbf{5}_{3}+\overline{\mathbf{5}}_{-3}$ & $I_{6}^{(0|| \mid 1)}$ \\
\hline
\end{tabular}

Table 1. Matter loci and U(1)-charges for the $I^{(0 \| 1)}$ fiber.

The $I_{5}$ singular fiber enhances to $I_{6}$ along $-a_{53}=a_{420}=0$ and $\left|\begin{array}{cc}a_{4} & -a_{5} \\ a_{0}+a_{3} & a_{3}\end{array}\right|=0$, and we have $I_{1}^{*}$ enhancement along $a_{5}=0$.

The sections intersect the $I_{5}$ fiber along $D_{0}$ and $D_{2}$, where, as above, the rational curves in the $I_{5}$ associated to the simple roots $\alpha_{i}$ are denoted by $D_{i}$, with the extended node corresponding to $D_{0}$. This type of $I_{5}$ fiber with two rational sections was denoted by $I_{5}^{(0|| 1)}$, where the separation of the sections is \#|-1. [34, 35]

\section{A.2 Other models with similar fiber type}

A different set of $I_{5}^{(0|| 1)}$ non-canonical models were determined in [34]

This model is based on the $I_{4}$ fiber $Q(3,2,1,0,0,0,1)$ given by

$$
\begin{array}{r}
c_{0} z^{3} w^{4}+c_{1} z^{2} w^{3} x+c_{2} z w^{2} x^{2}+c_{3} z w x^{3} \\
=y^{2}+b_{0} x^{2} y+b_{1} y w x+b_{2} z w^{2} y .
\end{array}
$$

where in addition we impose

$$
P_{0}=\left|\begin{array}{ll}
b_{0} & b_{2} \\
c_{3} & c_{2}
\end{array}\right|=0
$$

This last is solved as follows:

$$
\left|\begin{array}{ll}
b_{0} & b_{2} \\
c_{3} & c_{2}
\end{array}\right|=\left|\left(\begin{array}{l}
\sigma_{1} \\
\sigma_{4}
\end{array}\right)\left(\begin{array}{ll}
\sigma_{2} & \sigma_{3}
\end{array}\right)\right|=0 .
$$

This fibration $\left.Q(3,2,1,1,0,0,1)\right|_{P_{0}=0}$ has generically $I_{5}$ fiber, with same intersection pattern with the two rational sections as $W_{4}$, but it provides more matter loci, shown in the table, with the following expressions for the matter curves

$$
\begin{aligned}
P_{1}= & \sigma_{4} b_{2,1}^{2}+\sigma_{1}^{2} \sigma_{3} c_{0,3}-\sigma_{1} b_{2,1} c_{1,2} \\
P_{2}= & \sigma_{1} \sigma_{2}^{2}\left|\begin{array}{ll}
\sigma_{1} & b_{2,1} \\
\sigma_{4} & c_{1,2}
\end{array}\right|+\sigma_{1} \sigma_{3}^{2}\left|\begin{array}{ll}
\sigma_{1} & b_{0,1} \\
\sigma_{4} & c_{3,2}
\end{array}\right| \\
& +\sigma_{3} \sigma_{2}\left(\sigma_{4} \sigma_{1} b_{1,1}-\sigma_{1}^{2} c_{2,2}+\sigma_{4}^{2}\right) .
\end{aligned}
$$

The sections are not symmetric in the form above, but this can again be remedied by shifting as in (A.2). 
Open Access. This article is distributed under the terms of the Creative Commons Attribution License (CC-BY 4.0), which permits any use, distribution and reproduction in any medium, provided the original author(s) and source are credited.

\section{References}

[1] A. Anandakrishnan and S. Raby, SU(6) GUT Breaking on a Projective Plane, Nucl. Phys. B 868 (2013) 627 [arXiv:1205.1228] [INSPIRE].

[2] L.B. Anderson, J. Gray, A. Lukas and E. Palti, Two Hundred Heterotic Standard Models on Smooth Calabi-Yau Threefolds, Phys. Rev. D 84 (2011) 106005 [arXiv:1106.4804] [INSPIRE].

[3] L.B. Anderson, J. Gray, A. Lukas and E. Palti, Heterotic Line Bundle Standard Models, JHEP 06 (2012) 113 [arXiv:1202.1757] [INSPIRE].

[4] R. Barbieri, T. Gregoire and L.J. Hall, Mirror world at the large hadron collider, hep-ph/0509242 [INSPIRE].

[5] C. Beasley, J.J. Heckman and C. Vafa, GUTs and Exceptional Branes in F-theory - I, JHEP 01 (2009) 058 [arXiv:0802.3391] [INSPIRE].

[6] C. Beasley, J.J. Heckman and C. Vafa, GUTs and Exceptional Branes in F-theory - II: Experimental Predictions, JHEP 01 (2009) 059 [arXiv:0806.0102] [INSPIRE].

[7] Z.G. Berezhiani, Astrophysical implications of the mirror world with broken mirror parity, Acta Phys. Polon. B 27 (1996) 1503 [hep-ph/9602326] [InSPIRE].

[8] R. Blumenhagen, V. Braun, T.W. Grimm and T. Weigand, GUTs in Type IIB Orientifold Compactifications, Nucl. Phys. B $\mathbf{8 1 5}$ (2009) 1 [arXiv:0811.2936] [INSPIRE].

[9] R. Blumenhagen, T.W. Grimm, B. Jurke and T. Weigand, Global F-theory GUTs, Nucl. Phys. B 829 (2010) 325 [arXiv:0908.1784] [INSPIRE].

[10] R. Blumenhagen, Gauge Coupling Unification in F-theory Grand Unified Theories, Phys. Rev. Lett. 102 (2009) 071601 [arXiv:0812.0248] [INSPIRE].

[11] E. Brieskorn, Singular elements of semi-simple algebraic groups, in proceedings of the Congrès International des Mathématiciens, Nice, France, 1-10 Septembre 1970, in Actes du Congrès International des Mathématiciens. Tome 2, Gauthier-Villars, Paris France (1971), pp. $279-284$.

[12] F. Catanese, Automorphisms of rational double points and moduli spaces of surfaces of general type, Compos. Math. 61 (1987) 81.

[13] Z. Chacko, H.-S. Goh and R. Harnik, The Twin Higgs: Natural electroweak breaking from mirror symmetry, Phys. Rev. Lett. 96 (2006) 231802 [hep-ph/0506256] [INSPIRE].

[14] H. Clemens and S. Raby, Heterotic/F-theory Duality and Narasimhan-Seshadri Equivalence, arXiv: 1906.07238 [INSPIRE].

[15] H. Clemens and S. Raby, F-theory over a Fano threefold built from $A_{4}$-roots, arXiv: 1908.01110 [INSPIRE].

[16] R. Davies, Dirac gauginos and unification in F-theory, JHEP 10 (2012) 010 [arXiv:1205.1942v3] [INSPIRE]. 
[17] S. Dimopoulos, S. Raby and F. Wilczek, Supersymmetry and the Scale of Unification, Phys. Rev. D 24 (1981) 1681 [INSPIRE].

[18] S. Dimopoulos and H. Georgi, Softly Broken Supersymmetry and SU(5), Nucl. Phys. B 193 (1981) 150 [inSPIRE].

[19] M.J. Dolan, J. Marsano and S. Schäfer-Nameki, Unification and Phenomenology of F-theory GUTs with $\mathrm{U}(1)_{P Q}, J H E P 12$ (2011) 032 [arXiv: 1109.4958] [INSPIRE].

[20] R. Donagi and M. Wijnholt, Model Building with F-theory, Adv. Theor. Math. Phys. 15 (2011) 1237 [arXiv:0802.2969] [InSPIRE].

[21] R. Donagi and M. Wijnholt, Breaking GUT Groups in F-theory, Adv. Theor. Math. Phys. 15 (2011) 1523 [arXiv:0808.2223v2] [INSPIRE].

[22] R. Donagi, Y.-H. He, B.A. Ovrut and R. Reinbacher, The Spectra of heterotic standard model vacua, JHEP 06 (2005) 070 [hep-th/0411156] [INSPIRE].

[23] R. Friedman, J. Morgan and E. Witten, Vector bundles and F-theory, Commun. Math. Phys. 187 (1997) 679 [hep-th/9701162] [INSPIRE].

[24] T.W. Grimm and T. Weigand, On Abelian Gauge Symmetries and Proton Decay in Global F-theory GUTs, Phys. Rev. D 82 (2010) 086009 [arXiv:1006.0226] [INSPIRE].

[25] T.W. Grimm, M. Kerstan, E. Palti and T. Weigand, Massive Abelian Gauge Symmetries and Fluxes in F-theory, JHEP 12 (2011) 004 [arXiv: 1107.3842] [INSPIRE].

[26] H. Hayashi, R. Tatar, Y. Toda, T. Watari and M. Yamazaki, New Aspects of Heterotic-F Theory Duality, Nucl. Phys. B 806 (2009) 224 [arXiv:0805.1057] [INSPIRE].

[27] A. Hebecker and M. Trapletti, Gauge unification in highly anisotropic string compactifications, Nucl. Phys. B 713 (2005) 173 [hep-th/0411131] [INSPIRE].

[28] A. Hebecker and J. Unwin, Precision Unification and Proton Decay in F-theory GUTs with High Scale Supersymmetry, JHEP 09 (2014) 125 [arXiv: 1405.2930] [INSPIRE].

[29] L.E. Ibáñez and G.G. Ross, Low-Energy Predictions in Supersymmetric Grand Unified Theories, Phys. Lett. B 105 (1981) 439 [INSPIRE].

[30] V. Kac, Infinite-dimensional Lie algebras, Cambridge University Press, Cambridge U.K. (1990).

[31] Y. Kawamata and Y. Namikawa, Logarithmic deformations of normal crossing varieties and smoothing of degenerate Calabi-Yau varieties, Invent. Math. 118 (1994) 395.

[32] J.E. Kim, J.-H. Kim and B. Kyae, Superstring standard model from $\mathbb{Z}_{12-I}$ orbifold compactification with and without exotics and effective R-parity, JHEP 06 (2007) 034 [hep-ph/0702278] [INSPIRE].

[33] S. Krippendorf, H.P. Nilles, M. Ratz and M.W. Winkler, Hidden SUSY from precision gauge unification, Phys. Rev. D 88 (2013) 035022 [arXiv: 1306.0574] [INSPIRE].

[34] M. Kuntzler and S. Schäfer-Nameki, Tate Trees for Elliptic Fibrations with Rank one Mordell-Weil group, arXiv:1406.5174 [INSPIRE].

[35] C. Lawrie, S. Schäfer-Nameki and J.-M. Wong, F-theory and All Things Rational: Surveying U(1) Symmetries with Rational Sections, JHEP 09 (2015) 144 [arXiv: 1504.05593] [INSPIRE]. 
[36] O. Lebedev et al., A Mini-landscape of exact MSSM spectra in heterotic orbifolds, Phys. Lett. B 645 (2007) 88 [hep-th/0611095] [INSPIRE].

[37] O. Lebedev, H.P. Nilles, S. Ramos-Sanchez, M. Ratz and P.K.S. Vaudrevange, Heterotic mini-landscape (II): Completing the search for MSSM vacua in a $\mathbb{Z}_{6}$ orbifold, Phys. Lett. B 668 (2008) 331 [arXiv:0807.4384] [InSPIRE].

[38] O. Lebedev et al., The Heterotic Road to the MSSM with $R$ parity, Phys. Rev. D 77 (2008) 046013 [arXiv: 0708.2691] [INSPIRE].

[39] H.M. Lee et al., A unique $\mathbb{Z}_{4}$ R-symmetry for the MSSM, Phy. Lett. B 694 (2011) 491 [arXiv: 1009.0905] [INSPIRE].

[40] H.M. Lee et al., Discrete $R$ symmetries for the MSSM and its singlet extensions, Nucl. Phys. B 850 (2011) 1 [arXiv:1102.3595] [INSPIRE].

[41] E. Looijenga, Root Systems And Elliptic Curves, Invent. Math. 38 (1977) 17.

[42] J. Marsano and S. Schäfer-Nameki, Yukawas, G-flux and Spectral Covers from Resolved Calabi-Yau's, JHEP 11 (2011) 098 [arXiv:1108.1794] [INSPIRE].

[43] J. Marsano, H. Clemens, T. Pantev, S. Raby and H.-H. Tseng, A Global SU(5) F-theory model with Wilson line breaking, JHEP 01 (2013) 150 [arXiv:1206.6132] [INSPIRE].

[44] C. Mayrhofer, E. Palti and T. Weigand, Hypercharge Flux in IIB and F-theory: Anomalies and Gauge Coupling Unification, JHEP 09 (2013) 082 [arXiv:1303.3589] [INSPIRE].

[45] D.R. Morrison and D.S. Park, F-Theory and the Mordell-Weil Group of Elliptically-Fibered Calabi-Yau Threefolds, JHEP 10 (2012) 128 [arXiv:1208.2695] [INSPIRE].

[46] D. Mumford, Theta characteristics of an algebraic curve, Ann. Sci. Éc. Norm. Supér. 4 (1971) 181.

[47] D. Mumford, Prym Varieties I, in Contributions to Analysis: A collection of papers dedicated to Lipman Bers, Academic Press (1974), pp. 325-350.

[48] L.B. Okun, Mirror particles and mirror matter: 50 years of speculations and search, Phys. Usp. 50 (2007) 380 [hep-ph/0606202] [INSPIRE].

[49] S. Raby, M. Ratz and K. Schmidt-Hoberg, Precision gauge unification in the MSSM, Phys. Lett. B 687 (2010) 342 [arXiv:0911.4249] [INSPIRE].

[50] G.G. Ross, Wilson line breaking and gauge coupling unification, hep-ph/0411057 [INSPIRE].

[51] P. Slodowy, Simple singularities and simple algebraic groups, Springer-Verlag, Lect. Notes Math. 815 (1980) 1.

[52] R. Tatar, Y. Tsuchiya and T. Watari, Right-handed Neutrinos in F-theory Compactifications, Nucl. Phys. B 823 (2009) 1 [arXiv:0905.2289] [INSPIRE].

[53] M. Trapletti, Gauge symmetry breaking in orbifold model building, Mod. Phys. Lett. A 21 (2006) 2251 [hep-th/0611030] [INSPIRE]. 